\title{
Analytical Approach for the Systematic Research of the Periodic Ferroresonant Solutions in the Power Networks
}

\author{
Fathi Ben Amar ${ }^{1,2}$, Rachid Dhifaoui ${ }^{2}$ \\ ${ }^{1}$ Department of Technology, Preparatory Institute for Engineering Studies of Sfax (IPEIS), Sfax, Tunisia \\ ${ }^{2}$ Electric Networks and Machines Research Unit, Insat, Tunisia \\ E-mail:Fathi.Benamar@ipeis.rnu.tn, Rachid.Dhifaoui@insat.rnu.tn \\ Received June 7, 2011; revised July 17, 2011; accepted July 24, 2011
}

\begin{abstract}
Ferroresonance is a complex and little known electrotechnical phenomenon. This lack of knowledge means that it is voluntarily considered responsible for a number of unexplained destructions or malfunctioning of equipment. The mathematical framework most suited to the general study of this phenomenon is the bifurcation theory, the main tool of which is the continuation method. Nevertheless, the use of a continuation process is not devoid of difficulties. In fact, to continue the solutions isolats which are closed curves, it is necessary to know a solution belonging to this isolated curve (isolat) to initialise the continuation method. The principal contribution of this article is to develop an analytical method allowing systematic calculation of this initial solution for various periodic ferroresonant modes (fundamental, harmonic and subharmonic) appearing on nonlinear electric system. The approach proposed uses a problem formulation in the frequency domain. This method enables to directly determine the solution in steady state without computing of the transient state. When we apply this method to the single-phase ferroresonant circuits (series and parallels configurations), we could easily calculate an initial solution for each ferroresonant mode that can be established. Knowing this first solution, we show how to use this analytical approach in a continuation technique to find the other solutions. The totality of the obtained solutions is represented in a plane where the abscissa is the amplitude of the supply voltage and the ordinate the amplitude of the system's state variable (flux or voltage). The curve thus obtained is called "bifurcation diagram". We will be able to then obtain a synthetic knowledge of the possible behaviors of the two circuits and particularly the limits of the dangerous zones of the various periodic ferroresonant modes that may appear. General results related to the series ferroresonance and parallel ferroresonance, obtained numerically starting from the theoretical and real cases, are illustrated and discussed.
\end{abstract}

Keywords: Transformer, Ferroresonance, Fundamental Mode, Harmonic Mode, Subharmonic Mode, Fractional Mode, Isolat, Limit Point, Bifurcation, Dangerous Zone

\section{Introduction}

The ferroresonance is a nonlinear resonance phenomenon that can affect the electrical transmission and distribution networks. It indicates all oscillating phenomena, generally periodic, which particularly appear on all networks at capacitive dominant (single-phase or polyphase) in interaction with a ferromagnetic nonlinear element (unloaded transformer).

This phenomenon is characterized by the possibility of existence of several stable steady states for a given configuration and parameter values. These various operating modes, except the normal state, are obviously undesirable. They lead indeed either to out-of-tolerance over- voltages with values several times in excess of the network nominal voltage (case of fundamental or harmonic ferroresonance), or to overcurrents not less dangerous for the material (case of subharmonic ferroresonance) [1-4].

Modeling a ferroresonant circuit leads to a set of nonlinear differential Equations depending on various physical parameters. The mathematical framework adapted to the study of these dynamic systems is the bifurcation theory [4-7]. The essential tool within this framework is the continuation method. It makes it possible to obtain a global view of the phenomenon and to answer to concrete problems faced by the power system operator.

However, in order that this continuation method can function correctly, we must have an initial solution be- 
longing to the type of studied mode (fundamental, harmonic or subharmonic). This difficulty is particularly obvious in the case of the follow-up of a solutions isolat: no trivial solution is available to start the continuation. We thus need a systematic method of determination of this initial solution for the various ferroresonant modes [6-8].

In this study, we are interested uniquely in permanent periodic solutions (fundamental, harmonics and subharmonics) in single-phase. Our objective is to develop an analytical approach which enables us to solve the initialization problem, on the one hand, in the iterative calculation of the Galerkin method (very effective method to detect the occurrence conditions of the permanent ferroresonance) [4-7] and, on the other hand, in the continuation techniques used in the bifurcation methods.

At the beginning, we present the studied ferroresonant system, which corresponds to a series or parallel single-phase circuit, in mathematical form. We carry out a change of variables in order to simplify the study and to obtain a system of normalized Equations with a reduced number of unknowns. Then, we present the systematic stages of calculation of the various types of periodic modes, solutions of the standardized system. Finally, we show that this approach can be used in a continuation technique, which enables to give information on the existence limits of phenomenon when a parameter varies. We detail the influence of the supply voltage and the losses.

Based on real and theoretical circuit cases, we show the wealth of information to acquire by this analytical approach. Several results of continuation (bifurcation diagrams) concerning the fundamental mode, harmonics 3,5 and 7 modes and subharmonics 3, 5, 7, 9 and 5/3 modes, are presented. That enables us to provide a global vision of the phenomena and their occurrence zones.

\section{Presentation of the Proposed Analytical Method}

As the solutions in steady state are generally periodic, one seeks a formulation in frequency mode of the problem. The proposed analytical approach applies the same principle used in the Galerkin method. The idea is to seek a periodic solution in the form of Fourier series. For that, a circuit modeling is adopted, without the nonlinear element, based on the equivalent Thevenin model (Figure 1).

The magnetic characteristic $i(\varphi)$ of nonlinear inductance, often modelling an unloaded transformer, constitutes the most significant point in the occurrence of ferroresonance. It is described by the following univocal

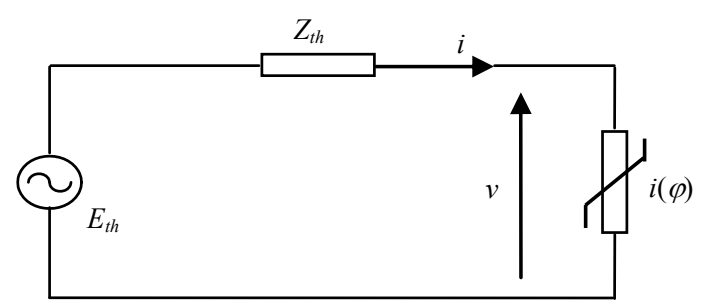

Figure 1. Equivalent study circuit.

relation between the current $i$ and flux $\varphi$ :

$$
i(\varphi)=a \cdot \varphi+b \cdot \varphi^{2 q+1} ; \quad q \in \mathbb{N},(a, b) \in \mathbb{R}^{+}
$$

This is an approximate but satisfactory representation permitting the study of ferroresonance.

For each frequency $k$, the complex Equation of the circuit (Figure 1) will be written as follows:

$$
j \omega_{k} \phi_{k}+Z_{k} I_{k}-E_{k}=0
$$

where $\omega_{k}$ is the pulsation at frequency $k, \phi_{k}, I_{k}, E_{k}$ and $Z_{k}$ are the complex components of the flux, current, supply voltage and Thevenin equivalent impedance at the harmonic frequency $k$.

In simple cases, it is possible to give an analytical expression to the relations of the current harmonic components $I_{k}$ in function of those of flow $\phi_{k}$. Such a procedure can be appropriate if the harmonic rate of the periodic modes is limited to the signals having only one or two spectral components. The Equations are then sufficiently simple so that one can analytically find a solution. It is even possible in this case to find all the problem solutions. These simplified solutions can be used to initialize the Galerkin method and the continuation techniques used in the bifurcation methods [4-7].

\subsection{Equivalent Circuit Equations}

Considering $\varphi(t)$ an approximate solution limited at order 2:

$$
\phi(t)=\varphi_{1} \cos (\omega t-\alpha)+\varphi_{h} \cos (h \omega t-\beta) ; \quad h \neq 1
$$

where $\omega$ is the pulsation at $50 \mathrm{~Hz}$ of the excitation. Except the fundamental ferroresonance, the row $h$ can be a multiple of the unit (case of the harmonic ferroresonance) or a fraction of this one (case of the subharmonic ferroresonance).

The Equation (2) is converted into a nonlinear algebraic system of 4 Equations, as follows:

$$
\begin{gathered}
h \omega \phi_{h c}-R_{h} I_{h s}+X_{h} I_{h c}=0 \\
h \omega \phi_{h s}+R_{h} I_{h c}+X_{h} I_{h s}=0 \\
\omega \phi_{1 c}-R_{1} I_{1 s}+X_{1} I_{1 c}=-E_{1 s} \\
\omega \phi_{1 s}+R_{1} I_{1 c}+X_{1} I_{1 s}=E_{1 c}
\end{gathered}
$$


with:

$$
\begin{aligned}
& \varphi_{h_{c}}=\varphi_{h} \cos \beta ; \quad \varphi_{h s}=\varphi_{h} \sin \beta ; \\
& \varphi_{1 c}=\varphi_{1} \cos \alpha ; \quad \varphi_{1 s}=\varphi_{1} \sin \alpha ; \\
& E_{1 c}=e_{1} \cdot E ; \quad E_{1 s}=e_{2} \cdot E .
\end{aligned}
$$

where $e_{1}$ and $e_{2}$ are the coefficients of the Thevenin equivalent voltage. The harmonic components of the current are calculated from (1); they are expressed in function of those of flux. The obtained system (4) has four Equations with four unknown variables $\left(\phi_{1}, \phi_{h}, \alpha\right.$ and $\beta$ ). In order to limit this number of unknowns, it is interesting to normalize this system.

\subsection{Normalization of the System}

To determine a particular solution of a system periodic mode (4), it is preferable to transform it as follows: by adding member to member the Equation (4a) multiplied by $\cos \beta$ and the Equation (4b) multiplied by $\sin \beta$, we obtain the Equation (5a). By subtracting member to member the Equation (4a) multiplied by $\sin \beta$ and the Equation (4b) multiplied by $\cos \beta$, we obtain the Equation $(5 b)$. We proceed in the same way for the Equations (4c) and (4d) but in multiplying them by $\cos \alpha$ and $\sin \alpha$, we thus obtain the Equations (5c) and (5d). The Equations system (4) becomes:

$$
\begin{gathered}
h \omega \phi_{h}+R_{h} G_{1}\left(\phi_{1}, \phi_{h}, \alpha, \beta\right)+X_{h} G_{2}\left(\phi_{1}, \phi_{h}, \alpha, \beta\right)=0 \\
R_{h} G_{2}\left(\phi_{1}, \phi_{h}, \alpha, \beta\right)-X_{h} G_{1}\left(\phi_{1}, \phi_{h}, \alpha, \beta\right)=0 \\
\omega \phi_{1}+R_{1} G_{3}\left(\phi_{1}, \phi_{h}, \alpha, \beta\right)+X_{1} G_{4}\left(\phi_{1}, \phi_{h}, \alpha, \beta\right) \\
=E \sqrt{e_{1}^{2}+e_{2}^{2}} \sin (\alpha+\theta) \\
R_{1} G_{4}\left(\phi_{1}, \phi_{h}, \alpha, \beta\right)-X_{1} G_{3}\left(\phi_{1}, \phi_{h}, \alpha, \beta\right) \\
E \sqrt{e_{1}^{2}+e_{2}^{2}} \cos (\alpha+\theta)
\end{gathered}
$$

with:

$$
\begin{aligned}
& G_{1}\left(\phi_{1}, \phi_{h}, \alpha, \beta\right)=I_{h c}\left(\phi_{1}, \phi_{h}, \alpha, \beta\right) \cdot \sin \beta \\
&-I_{h s}\left(\phi_{1}, \phi_{h}, \alpha, \beta\right) \cdot \cos \beta \\
& G_{2}\left(\phi_{1}, \phi_{h}, \alpha, \beta\right)= I_{h c}\left(\phi_{1}, \phi_{h}, \alpha, \beta\right) \cdot \cos \beta \\
&+I_{h s}\left(\phi_{1}, \phi_{h}, \alpha, \beta\right) \cdot \sin \beta \\
& G_{3}\left(\phi_{1}, \phi_{h}, \alpha, \beta\right)=I_{1 c}\left(\phi_{1}, \phi_{h}, \alpha, \beta\right) \cdot \sin \alpha \\
&-I_{1 s}\left(\phi_{1}, \phi_{h}, \alpha, \beta\right) \cdot \cos \alpha \\
& G_{4}\left(\phi_{1}, \phi_{h}, \alpha, \beta\right)=I_{1 c}\left(\phi_{1}, \phi_{h}, \alpha, \beta\right) \cdot \cos \alpha \\
&+I_{1 s}\left(\phi_{1}, \phi_{h}, \alpha, \beta\right) \cdot \sin \alpha \\
& \tan \theta=-\frac{e_{2}}{e_{1}}
\end{aligned}
$$

This system (5) can still be written in the following form:

$$
\begin{aligned}
& h \omega \phi_{h}+R_{h} G_{1}\left(\phi_{1}, \phi_{h}, \alpha, \beta\right) \\
& +X_{h} G_{2}\left(\phi_{1}, \phi_{h}, \alpha, \beta\right)=0 \\
& R_{h} G_{2}\left(\phi_{1}, \phi_{h}, \alpha, \beta\right)-X_{h} G_{1}\left(\phi_{1}, \phi_{h}, \alpha, \beta\right)=0 \\
& \tan (\alpha+\theta) \\
& =\frac{\omega \phi_{1}+R_{1} G_{3}\left(\phi_{1}, \phi_{h}, \alpha, \beta\right)+X_{1} G_{4}\left(\phi_{1}, \phi_{h}, \alpha, \beta\right)}{R_{1} G_{4}\left(\phi_{1}, \phi_{h}, \alpha, \beta\right)-X_{1} G_{3}\left(\phi_{1}, \phi_{h}, \alpha, \beta\right)} \\
& {\left[\omega \phi_{1}+R_{1} G_{3}\left(\phi_{1}, \phi_{h}, \alpha, \beta\right)+X_{1} G_{4}\left(\phi_{1}, \phi_{h}, \alpha, \beta\right)\right]^{2}} \\
& +\left[R_{1} G_{4}\left(\phi_{1}, \phi_{h}, \alpha, \beta\right)-X_{1} G_{3}\left(\phi_{1}, \phi_{h}, \alpha, \beta\right)\right]^{2} \\
& =E^{2}\left(e_{1}^{2}+e_{2}^{2}\right)
\end{aligned}
$$

In order to reduce the number of unknowns in (6), it is necessary to carry out the following change of variable:

$$
Q=\frac{\varphi_{h}}{\varphi_{1}} \quad \text { avec } \quad Q \in \mathfrak{R}^{*}
$$

which enables to write the system (6) in a normalized general form (8):

$$
\begin{aligned}
& h \omega Q \phi_{1}+R_{h} \tilde{G}_{1}\left(Q, \phi_{1}, \alpha, \beta\right)+X_{h} \tilde{G}_{2}\left(Q, \phi_{1}, \alpha, \beta\right)=0 \\
& R_{h} \tilde{G}_{2}\left(Q, \phi_{1}, \alpha, \beta\right)-X_{h} \tilde{G}_{1}\left(Q, \phi_{1}, \alpha, \beta\right)=0 \\
& \tan (\alpha+\theta) \\
& =\frac{\omega \phi_{1}+R_{1} \tilde{G}_{3}\left(Q, \phi_{1}, \alpha, \beta\right)+X_{1} \tilde{G}_{4}\left(Q, \phi_{1}, \alpha, \beta\right)}{R_{1} \tilde{G}_{4}\left(Q, \phi_{1}, \alpha, \beta\right)-X_{1} \tilde{G}_{3}\left(Q, \phi_{1}, \alpha, \beta\right)}
\end{aligned}
$$

which is solved easily.

With $\tilde{G}_{1}, \tilde{G}_{2}, \tilde{G}_{3}$ and $\tilde{G}_{4}$ are functions limited uniquely to the three unknowns $\phi_{1}, \alpha$ and $\tilde{\beta}$. This enables us to find a solution of the studied mode.

To find all the corresponding approximate solutions to a given set of parameters of the study circuit or to approximately determine the existence zones of the various ferroresonant modes, it is then sufficient to iterate on the new variable $Q$.

This developed tool is general and can adapt to any type of circuit single-phase. Moreover, it constitutes an excellent means of search for an initial solution to include in the iterative calculation of the Galerkin method, particularly in the case of follow-up of solutions isolat.

In order to explain the implementation of this analytical method, we present it in detail in the following paragraph.

\section{Application to Study of Single-Phase Ferroresonance}

The ferroresonance can occur in single-phase or polyphase circuits. The circuit configurations under which 
this phenomenon can occur are endless [9-11]. We distinguish the configurations known as series (resp. parallel), where we find a capacity in series (resp. in parallel) with the nonlinear element and the source voltage.

Frequently, the encountered practical situations are generally three-phase but, thanks to an adapted modeling, they can be transformed into single-phase ferroresonance cases [2-4]. This is why we have chosen in our study a single-phase representation of the studied system (series or parallel type).

\subsection{Study of Parallel Ferroresonance}

We apply the proposed approach to the research of the periodic ferroresonant modes of the circuit of Figure 2, which enables to study the problems of the parallel ferroresonance. It is about a single-phase model of an operation of voltage restoration between a sinusoidal voltage source and an unloaded power transformer, through an underground cable or a long overhead line [10]. It is the capacity of the line which is the responsible element revealing the phenomenon.

The physical parameters of this circuit are:

$E$ : amplitude of the supply voltage $e(t)=E \cos (100 \pi t)$,

$C_{t}$ : equivalent capacitance of the circuit,

$L_{g}, L_{d}$ : linear inductances of the circuit,

$R_{g}, R_{d}$ : series losses of the circuit,

$R_{t}$ : parallel losses of the circuit.

The magnetic characteristic is defined by:

$i(\varphi)=1,84 \cdot 10^{-3} \varphi+61 \cdot 10^{-24} \varphi^{9}$. It corresponds to a real power transformer, single-phase, of 360 MVA and nominal primary voltage $130 \mathrm{kV}$.

By a judicious choice of the circuit parameters, we can determine the various possible modes of fundamental, harmonic and subharmonic ferroresonance [12-15].

\subsubsection{Search for Integer Subharmonics Modes}

In this case, the row $h$ is a simple fraction of the unit. To fix the ideas, we detail here the example of the subharmonic mode 3 ( $h=1 / 3$, we use the notation SH3). We choose the case where the study circuit parameters (Figure 2) are: $L_{g}=2.25 \mathrm{H} ; R_{g}=4 \Omega ; C_{t}=43.5 \mu \mathrm{F} ; R_{t}=0.1$ $\mathrm{M} \Omega ; L_{d}=0.1 \mathrm{H} ; R_{d}=1 \Omega$, which represent a theoretical case allowing the study of the SH3.

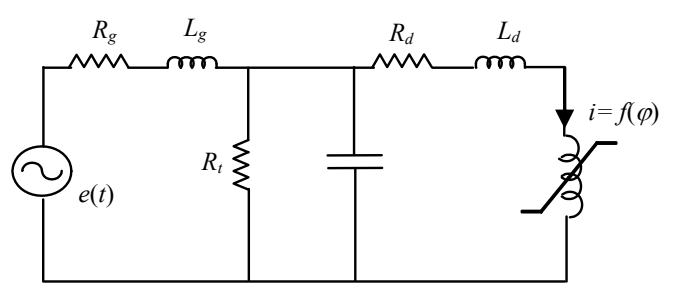

Figure 2. Parallel, single-phase, nonlinear ferroresonant circuit.
We start then by calculating the Fourier coefficients of the harmonic currents $I_{1}$ and $I_{1 / 3}$ by virtue of relation $i(\varphi)$. We obtain:

$$
\begin{aligned}
I_{\frac{1}{3} c} & =B_{1} \cos \psi_{1}+B_{2} \cos \psi_{2}+B_{3} \cos \psi_{3} \\
& +B_{4} \cos \psi_{4}+B_{5} \cos \psi_{5} \\
I_{\frac{1}{3} s} & =B_{1} \sin \psi_{1}+B_{2} \sin \psi_{2}+B_{3} \sin \psi_{3} \\
& +B_{4} \sin \psi_{4}+B_{5} \sin \psi_{5} \\
I_{1 c} & =B_{6} \cos \psi_{6}+B_{7} \cos \psi_{7}+B_{8} \cos \psi_{8} \\
& +B_{9} \cos \psi_{9}+B_{10} \cos \psi_{10} \\
I_{1 s}= & B_{6} \sin \psi_{6}+B_{7} \sin \psi_{7}+B_{8} \sin \psi_{8} \\
& +B_{9} \sin \psi_{9}+B_{10} \sin \psi_{10}
\end{aligned}
$$

with:$$
\begin{aligned}
B_{1}= & k_{1} \phi_{\frac{1}{3}}+\frac{b}{256}\left(126 \phi_{\frac{1}{3}}^{9}+630 \phi_{1}^{8} \phi_{\frac{1}{3}}+2520 \phi_{1}^{2} \phi_{\frac{1}{3}}^{7}\right. \\
& \left.+5040 \phi_{1}^{6} \phi_{\frac{1}{3}}^{3}+7560 \phi_{1}^{4} \phi_{\frac{1}{3}}^{5}\right)
\end{aligned}
$$$$
B_{2}=\frac{k_{9}}{256}\left(504 \phi_{1} \phi_{\frac{1}{3}}^{8}+1260 \phi_{1}^{7} \phi_{\frac{1}{3}}^{2}+3780 \phi_{1}^{3} \phi_{\frac{1}{3}}^{6}+5040 \phi_{1}^{5} \phi_{\frac{1}{3}}^{4}\right)
$$$$
B_{3}=\frac{k_{9}}{256}\left(252 \phi_{1} \phi_{\frac{1}{3}}^{8}+1512 \phi_{1}^{3} \phi_{\frac{1}{3}}^{6}+1260 \phi_{1}^{5} \phi_{\frac{1}{3}}^{4}\right)
$$$$
B_{4}=\frac{k_{9}}{256}\left(252 \phi_{1}^{2} \phi_{\frac{1}{3}}^{7}+504 \phi_{1}^{4} \phi_{\frac{1}{3}}^{5}\right)
$$$$
B_{5}=\frac{k_{9}}{256}\left(36 \phi_{1}^{2} \phi_{\frac{1}{3}}^{7}\right)
$$$$
B_{6}=k_{1} \phi_{1}+\frac{b}{256}\left(126 \phi_{1}^{9}+630 \phi_{1} \phi_{\frac{1}{3}}^{8}+2520 \phi_{1}^{7} \phi_{\frac{1}{3}}^{2}\right.
$$$$
\left.+5040 \phi_{1}^{3} \phi_{h}^{6}+7560 \phi_{1}^{5} \phi_{\frac{1}{3}}^{4}\right)
$$$$
B_{7}=\frac{k_{9}}{256}\left(84 \phi_{\frac{1}{3}}^{9}+1512 \phi_{1}^{2} \phi_{\frac{1}{3}}^{7}+1680 \phi_{1}^{6} \phi_{\frac{1}{3}}^{3}+3780 \phi_{1}^{4} \phi_{\frac{1}{3}}^{5}\right)
$$$$
B_{8}=\frac{k_{9}}{256}\left(756 \phi_{1}^{2} \phi_{\frac{1}{3}}^{7}+1260 \phi_{1}^{6} \phi_{\frac{1}{3}}^{3}+2520 \phi_{1}^{4} \phi_{\frac{1}{3}}^{5}\right)
$$$$
B_{9}=\frac{k_{9}}{256}\left(72 \phi_{1} \phi_{\frac{1}{3}}^{8}+252 \phi_{1}^{3} \phi_{\frac{1}{3}}^{6}\right)
$$$$
B_{10}=\frac{k_{9}}{256}\left(84 \phi_{1}^{3} \phi_{\frac{1}{3}}^{6}\right)
$$$$
\psi_{1}=\beta ; \quad \psi_{2}=\alpha-2 \beta ; \quad \psi_{3}=4 \beta-\alpha ; \quad \psi_{4}=2 \alpha-5 \beta ;
$$$$
\psi_{5}=7 \beta-2 \alpha ; \quad \psi_{6}=\alpha ; \quad \psi_{7}=3 \beta, \quad \psi_{8}=2 \alpha-3 \beta \text {; }
$$$$
\psi_{9}=6 \beta-\alpha ; \quad \psi_{10}=3 \alpha-6 \beta
$$ 
The system (4) is converted into:

$\frac{\omega}{3} \phi_{\frac{1}{3}} \cos \psi_{1}-R_{\frac{1}{3}}\left(B_{1} \sin \psi_{1}+B_{2} \sin \psi_{2}+B_{3} \sin \psi_{3}\right.$

$\left.+B_{4} \sin \psi_{4}+B_{5} \sin \psi_{5}\right)+X_{\frac{1}{3}}\left(B_{1} \cos \psi_{1}+B_{2} \cos \psi_{2}\right.$

$\left.+B_{3} \cos \psi_{3}+B_{4} \cos \psi_{4}+B_{5} \cos \psi_{5}\right)=0$

$\frac{\omega}{3} \phi_{\frac{1}{3}} \sin \psi_{1}+R_{\frac{1}{3}}\left(B_{1} \cos \psi_{1}+B_{2} \cos \psi_{2}+B_{3} \cos \psi_{3}\right.$

$\left.+B_{4} \cos \psi_{4}+B_{5} \cos \psi_{5}\right)+X_{\frac{1}{3}}\left(B_{1} \sin \psi_{1}+B_{2} \sin \psi_{2}\right.$

$\left.+B_{3} \sin \psi_{3}+B_{4} \sin \psi_{4}+B_{5} \sin \psi_{5}\right)=0$

$\omega \phi_{1} \cos \psi_{6}-R_{1}\left(B_{6} \sin \psi_{6}+B_{7} \sin \psi_{7}+B_{8} \sin \psi_{8}\right.$

$\left.+B_{9} \sin \psi_{9}+B_{10} \sin \psi_{10}\right)+X_{1}\left(B_{6} \cos \psi_{6}+B_{7} \cos \psi_{7}\right.$

$\left.+B_{8} \cos \psi_{8}+B_{9} \cos \psi_{9}+B_{10} \cos \psi_{10}\right)=-E_{1 s}$

$\omega \phi_{1} \sin \psi_{6}+R_{1}\left(B_{6} \cos \psi_{6}+B_{7} \cos \psi_{7}+B_{8} \cos \psi_{8}\right.$

$\left.+B_{9} \cos \psi_{9}+B_{10} \cos \psi_{10}\right)+X_{1}\left(B_{6} \sin \psi_{6}+B_{7} \sin \psi_{7}\right.$

$\left.+B_{8} \sin \psi_{8}+B_{9} \sin \psi_{9}+B_{10} \sin \psi_{10}\right)=E_{1 c}$

By adding the Equation (10a) multiplied by $\cos \psi_{1}$ and the Equation (10b) multiplied by $\sin \psi_{1}$, we obtain the Equation (11):

$$
\begin{aligned}
& \frac{\omega}{3} \phi_{\frac{1}{3}}+X_{\frac{1}{3}} B_{1}+\left(R_{\frac{1}{3}} B_{2}-R_{\frac{1}{3}} B_{3}\right) \sin \gamma \\
& +\left(R_{\frac{1}{3}} B_{4}-R_{\frac{1}{3}} B_{5}\right) \sin 2 \gamma+\left(X_{\frac{1}{3}} B_{2}+X_{\frac{1}{3}} B_{3}\right) \cos \gamma \\
& +\left(X_{\frac{1}{3}} B_{4}+X_{\frac{1}{3}} B_{5}\right) \cos 2 \gamma=0
\end{aligned}
$$

with $\gamma=3 \beta-\alpha$.

By subtracting the Equation (10a) multiplied by $\sin \psi_{1}$ from the Equation (10b) multiplied by $\cos \psi_{1}$, we obtain the Equation (12):

$$
\begin{aligned}
& R_{\frac{1}{3}} B_{1}+\left(R_{\frac{1}{3}} B_{2}+R_{\frac{1}{3}} B_{3}\right) \cos \gamma+\left(R_{\frac{1}{3}} B_{4}+R_{\frac{1}{3}} B_{5}\right) \cos 2 \gamma \\
& +\left(X_{\frac{1}{3}} B_{3}+X_{\frac{1}{3}} B_{2}\right) \sin \gamma+\left(X_{\frac{1}{3}} B_{5}-X_{\frac{1}{3}} B_{4}\right) \sin 2 \gamma=0
\end{aligned}
$$

We proceed in the same way for the Equations (10c) and (10d) but by multiplying them by $\cos \psi_{6}$ and $\sin \psi_{6}$, thus we obtain the Equations (13) and (14):

$$
\begin{aligned}
& \omega \phi_{1}+X_{1} B_{6}+\left(R_{1} B_{8}-R_{1} B_{7}\right) \sin \gamma \\
& +\left(R_{1} B_{10}-R_{1} B_{9}\right) \sin 2 \gamma+\left(X_{1} B_{7}+X_{1} B_{8}\right) \cos \gamma \\
& +\left(X_{1} B_{9}+X_{1} B_{10}\right) \cos 2 \gamma=E \sqrt{e_{1}^{2}+e_{2}^{2}} \sin \left(\psi_{6}+\theta\right)
\end{aligned}
$$

$$
\begin{aligned}
& R_{1} B_{6}+\left(R_{1} B_{7}+R_{1} B_{8}\right) \cos \gamma \\
& +\left(R_{1} B_{9}+R_{1} B_{10}\right) \cos 2 \gamma+\left(X_{1} B_{7}-X_{1} B_{8}\right) \sin \gamma \\
& +\left(X_{1} B_{9}-X_{1} B_{10}\right) \sin 2 \gamma=E \sqrt{e_{1}^{2}+e_{2}^{2}} \cos \left(\psi_{6}+\theta\right)
\end{aligned}
$$

Equations (11)-(14) constitute a system of four Equations in four unknowns $\phi_{1}, \phi_{1 / 3}, \alpha$ and $\beta$. To reduce the number of unknowns to three, we adopt the following change of variable:

$$
Q=\frac{\phi_{1}}{\phi_{1}} \quad Q \in R^{+}
$$

We show that, for a given value of $Q$, we can completely solve the Equations system (11) to (14) and to deduce an approximate solution from the complete system (2). The supply voltage amplitude $E$ is directly determined by the ratio between the two Equations (13) and (14). By varying $Q$, we thus compute all the solutions of the characteristic for $\mathrm{SH} 3$ mode in function of the source voltage $E$. By using the terminology of the bifurcation theory, the curve thus obtained in the plane, where the abscissa is the amplitude $E$ and the ordinate is the amplitude of the system's state variable (flux or voltage), is called "bifurcation diagram". Then we will be able to then get information on the existence zone of the mode studied in function of the voltage $E$ and to precise its critical values which correspond to limit point bifurcations [4-6].

By replacing the functions $B_{i(i=1 \text { à } 10)}$ by their expressions and $\phi_{1 / 3}$ per $Q \cdot \phi_{1}$ in the two Equations (11) and (12) where there is not source component, we obtain:

$$
\begin{aligned}
& \phi_{\frac{1}{3}}\left[\frac{\omega}{3}+k_{1} X_{\frac{1}{3}}+\frac{k_{9}}{256} \phi_{1}^{8}\left(X_{\frac{1}{3}} P_{1}+R_{\frac{1}{3}} P_{4} \sin \gamma\right.\right. \\
& \left.\left.+R_{\frac{1}{3}} P_{5} \sin 2 \gamma+X_{\frac{1}{3}} P_{2} \cos \gamma+X_{\frac{1}{3}} P_{3} \cos 2 \gamma\right)\right]=0 \\
& \phi_{\frac{1}{3}}\left[k_{1} R_{\frac{1}{3}}+\frac{k_{9}}{256} \phi_{1}^{8}\left(R_{\frac{1}{3}} P_{1}+R_{\frac{1}{3}} P_{2} \cos \gamma\right.\right. \\
& \left.\left.+R_{\frac{1}{3}} P_{3} \cos 2 \gamma-X_{\frac{1}{3}} P_{4} \sin \gamma-X_{\frac{1}{3}} P_{5} \sin 2 \gamma\right)\right]=0
\end{aligned}
$$

where the elements $P_{i(i=1 \text { a } 5)}$ are polynomials in $Q$ :

$$
\begin{aligned}
& P_{1}=126 Q^{8}+2520 Q^{6}+7560 Q^{4}+5040 Q^{2}+630 \\
& P_{2}=756 Q^{7}+5292 Q^{5}+6300 Q^{3}+1260 Q \\
& P_{3}=288 Q^{6}+504 Q^{4} \\
& P_{4}=252 Q^{7}+2268 Q^{5}+3780 Q^{3}+1260 Q \\
& P_{5}=216 Q^{6}+504 Q^{4}
\end{aligned}
$$

A first solution of the Equations (16) and (17) is $\phi_{1 / 3}=$ 
0 : it corresponds to the harmonic modes. To calculate the subharmonic modes, let us eliminate component $\frac{k_{9}}{256} \phi_{1}^{8}$ between the Equations (16) and (17). We obtain a relation in $\gamma$ of type:

$$
a \cdot \cos \gamma+b \cdot \sin \gamma+c \cdot \cos 2 \gamma+d \cdot \sin 2 \gamma=e
$$

with:

$$
\begin{aligned}
& a=-R_{\frac{1}{3}} \frac{\omega}{3} P_{2} \\
& b=X_{\frac{1}{3}} \frac{\omega}{3} P_{4}+X_{\frac{1}{3}}^{2} k_{1} P_{4}+k_{1} R_{\frac{1}{3}}^{2} P_{4} \\
& c=-R_{\frac{1}{3}} \frac{\omega}{3} P_{3} \\
& d=X_{\frac{1}{3}} \frac{\omega}{3} P_{5}+X_{\frac{1}{3}}^{2} k_{1} P_{5}+k_{1} R_{\frac{1}{3}}^{2} P_{5} \\
& e=R_{\frac{1}{3}} \frac{\omega}{3} P_{1}
\end{aligned}
$$

In order to solve relation (18), we make the following change of variables:

$$
\left\{\begin{array}{l}
x=\sin \gamma \\
y=\cos \gamma
\end{array} \text { tel que } x^{2}+y^{2}=1 \text { avec } x, y \in[-1,1]\right.
$$

which enables to write the relation (18) in form of a system of two nonlinear Equations to two unknowns ( $x$ and $y)$ :

$$
\left\{\begin{array}{l}
f(x, y)=2 c x^{2}-b x-a y-2 d x y-c+e=0 \\
g(x, y)=x^{2}+y^{2}-1=0
\end{array}\right.
$$

In order to determine whether the system admits solutions on $[-1,1]$, we will plot the representative curves of the two functions $f$ and $g$ in order to show the existence of a couple $(x, y)$ where they intersect. Let us note that $g(x, y)=0$ is the Equation of unit circle.

For a fixed value of $Q$ and under condition $x^{2}+y^{2}=1$ (condition which fixes the interval of $Q$ for which a SH3 mode exists), the tracing of the representative curves of the two functions $f$ and $g$ of the system (20) shows the existence of two intersection points $S_{1}\left(x_{1}, y_{1}\right)$ et $S_{2}\left(x_{2}, y_{2}\right)$ (Figure 3).

Knowing $\gamma$, the Equation (17) enables to calculate $\phi_{1}$. Then $\phi_{1 / 3}$ is given by the relation (15):

$$
\phi_{\frac{1}{3}}=Q \cdot \phi_{1}
$$

Parameter $\alpha$ is deduced by the ratio between the two

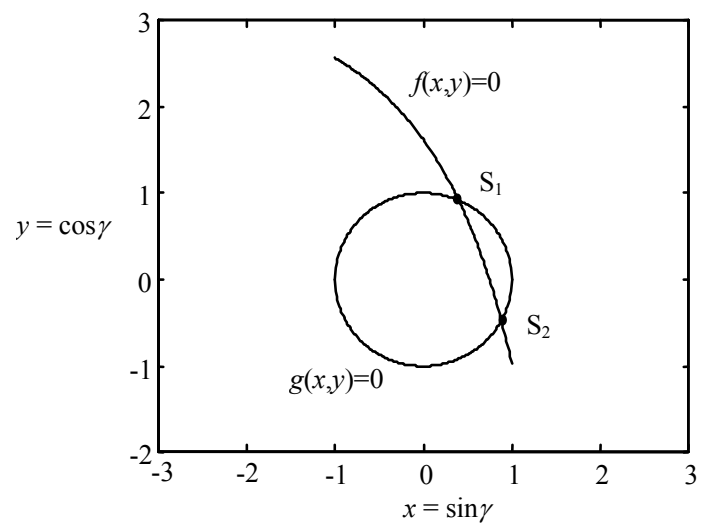

Figure 3. Intersection of the two representative curves of the functions $f(x, y)=0$ and $g(x, y)=0$.

Equations (13) and (14): (see Equation (21))

Knowing $\alpha$, the same Equations (13) and (14) also enable to calculate the amplitude of source voltage $E$.

Finally, the parameter $\beta$ is deduced starting from the relation (13):

$$
\beta=\frac{1}{3}(\gamma+\alpha)
$$

In this way, we can easily solve the initialization problem observed in the continuation procedures used in the bifurcation method, and in the iterative calculation procedure used in the Galerkin method. The latter method enables to calculate precisely the ferroresonant modes.

We present, in Figure 4, the solution waveforms obtained by the proposed approach (flux $\varphi(t)$ in the target transformer and the voltage $V(t)$ on its terminals), over a period, for the peak value of the nominal voltage $(E=$ $183 \mathrm{kV}$ ): it is about a solution of SH3 mode. Indeed, its basic period is three times as equal as the excitation period $T_{0}=20 \mathrm{~ms}$. It is then easy to note that, under the influence of the presence of the $1 / 3$ harmonic component, the solution waveform is obviously deformed.

From this solution, we vary progressively the parameter $Q$, the two intersection points and system (20) solutions. Then, we generate two $\mathrm{SH} 3$ solution branches which meet in two limit points $B P_{1}$ and $B P_{2}$ (Figure 5(a)), called bifurcation points for which there is stability change. Thus, we obtain a bifurcation diagram of the $\mathrm{SH} 3$ ferroresonant mode (noted DB-SH3). To have an overall view of the results, we present this bifurcation diagram in function of voltage $E$ for each variable $(\varphi, V$, $\left.\phi_{1}, V_{1}, \phi_{1 / 3}, V_{1 / 3}\right)$ : they are solution isolats (Figures 5(a) and (b)).

On the other hand, the diagram surface (in other words

$$
\tan (\alpha+\theta)=\frac{\omega \phi_{1}+X_{1} B_{6}+\left(R_{1} B_{8}-R_{1} B_{7}\right) \sin \gamma+\left(R_{1} B_{10}-R_{1} B_{9}\right) \sin 2 \gamma+\left(X_{1} B_{7}+X_{1} B_{8}\right) \cos \gamma+\left(X_{1} B_{9}+X_{1} B_{10}\right) \cos 2 \gamma}{R_{1} B_{6}+\left(R_{1} B_{7}+R_{1} B_{8}\right) \cos \gamma+\left(R_{1} B_{9}+R_{1} B_{10}\right) \cos 2 \gamma+\left(X_{1} B_{7}-X_{1} B_{8}\right) \sin \gamma+\left(X_{1} B_{9}-X_{1} B_{10}\right) \sin 2 \gamma}
$$



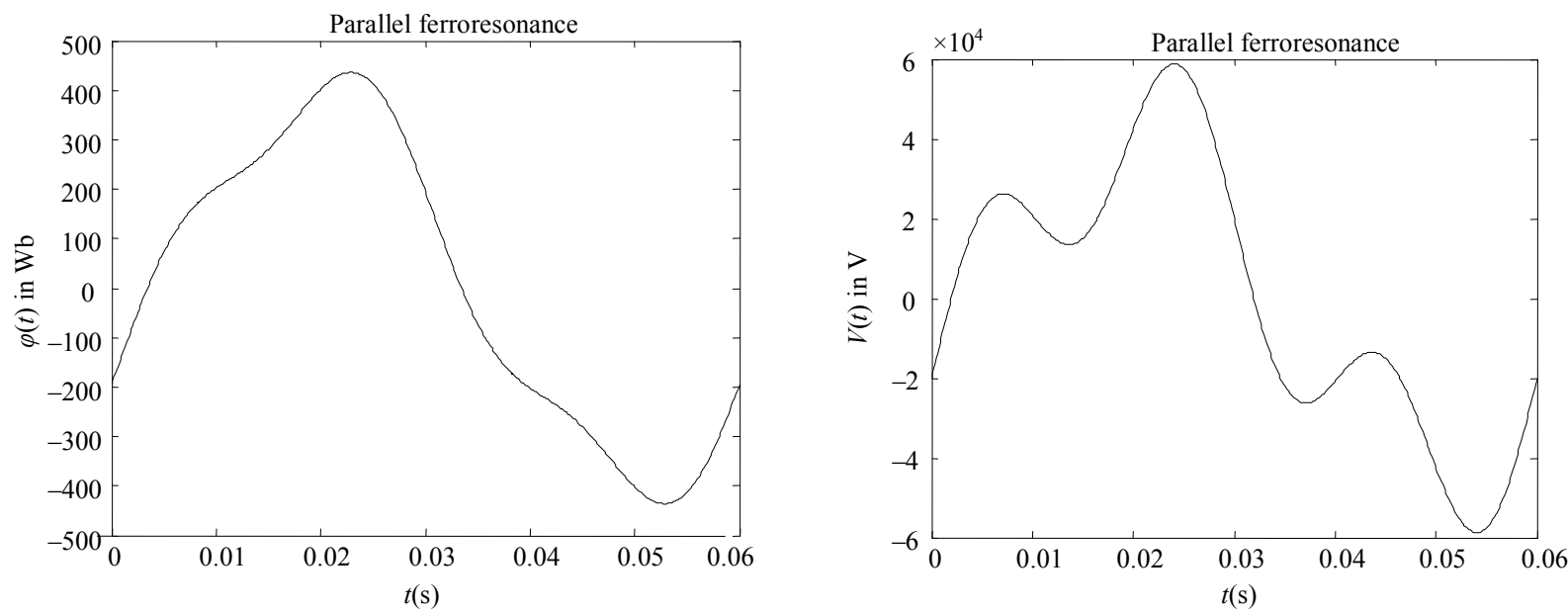

Figure 4. SH3 solution: Flux $\varphi(t)$ and voltage $V(t)$ waveforms on the target transformer during one period for the subharmonic-3 ferroresonance for $E=183 \mathrm{kV}$ and $R_{g}=4 \Omega$.

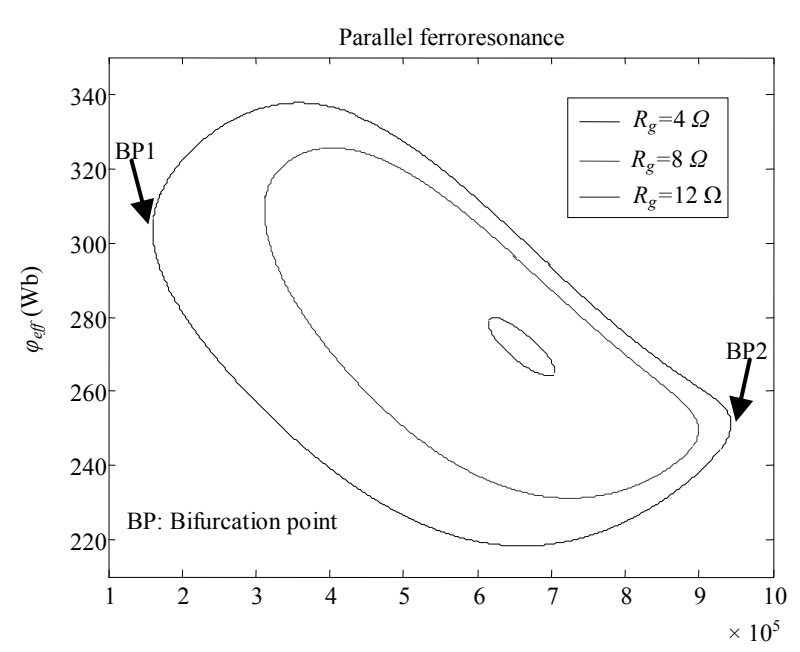

(a)

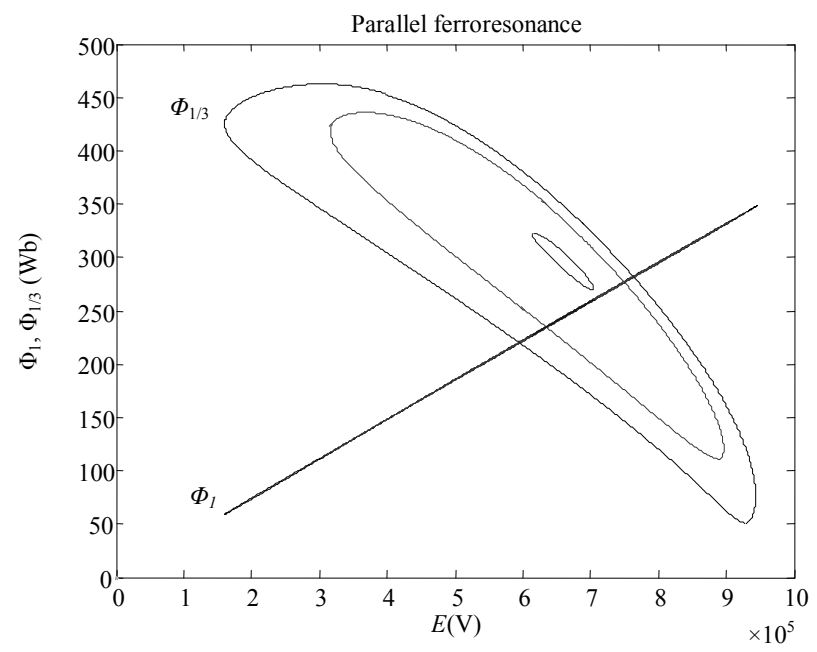

(c)

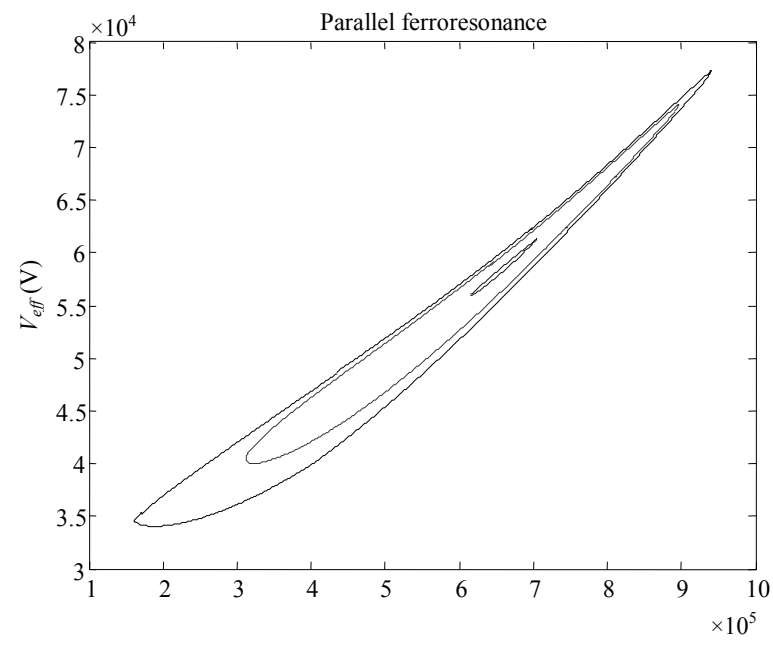

(b)

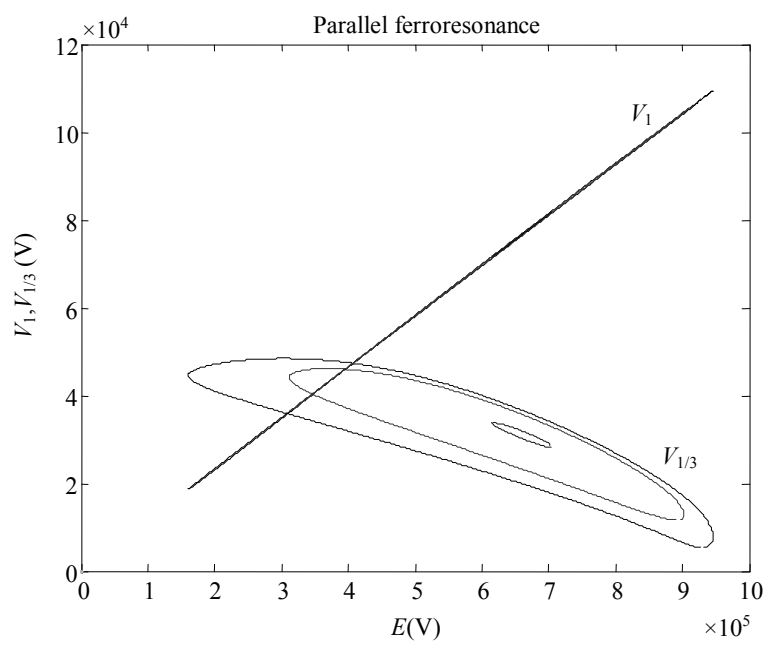

(d)

Figure 5. Bifurcation diagrams of SH3 mode as a function of $\mathrm{E}$, for various values of the series losses $R_{g}\left(L_{g}=2.25 \mathrm{H}, L_{d}=0.1 \mathrm{H}\right.$, $C_{t}=43.5 \mu \mathrm{F}, R_{t}=0.1 \mathrm{M} \Omega$ and $\left.R_{d}=1 \Omega\right)$. 
the existence zone of mode SH3) is more reduced when series losses $R_{g}$ of system are large. In addition, the minimum voltage of the existence of this ferroresonant mode (where the first bifurcation $B P_{1}$ ) increases. In contrast, the maximum voltage of existence (where the second bifurcation $\mathrm{BP}_{2}$ ) decreases. Indeed, in order that this mode persists, it is necessary that the source energy can compensate for the network losses [16,17]. This remains possible for strong voltages. But these voltages are not realistic for an electrical power network. The considerations above also apply to the other types of periodic ferroresonant modes.

Let us announce that, for certain values of $E$, the amplitude of SH3 component can exceed considerably the amplitude of the $50 \mathrm{~Hz}$ component (Figure 5(b)). In fact, the ratio $Q(15)$, which enables to relativize the $\mathrm{SH} 3$ compared to that $50 \mathrm{~Hz}$, has for value several times the unit.

The same reasoning is equally applied to calculate the other subharmonics modes. On Figures 6-11, we summarize the results of the proposed approach concerning modes SH5, SH7 and SH9 (these are theoretical cases). Qualitatively, the remarks previously quoted on the DB-SH3 are retained for the DB-SH5, DB-SH7 and DB-SH9.

On Figure 12, we compare the bifurcation diagrams of the subharmonics 3, 5, 7, 9 modes and the fundamental mode, which can exist simultaneously for the following theoretical case: $L_{g}=2.25 \mathrm{H}, R_{g}=1 \Omega, C_{t}=1100 \mu \mathrm{F}, R_{t}$ $=0.1 \mathrm{M} \Omega$ and $L_{d}=R_{d}=0$. We then conclude that:

- Contrary to the fundamental ferroresonance mode, the bifurcation diagrams of the subharmonics modes are solution isolats and no intersection with trivial solutions.

- The existence domains of these oscillation modes decrease when the row of subharmonic increases.

- The effective values reaching by the flux $\varphi(t)$ and the

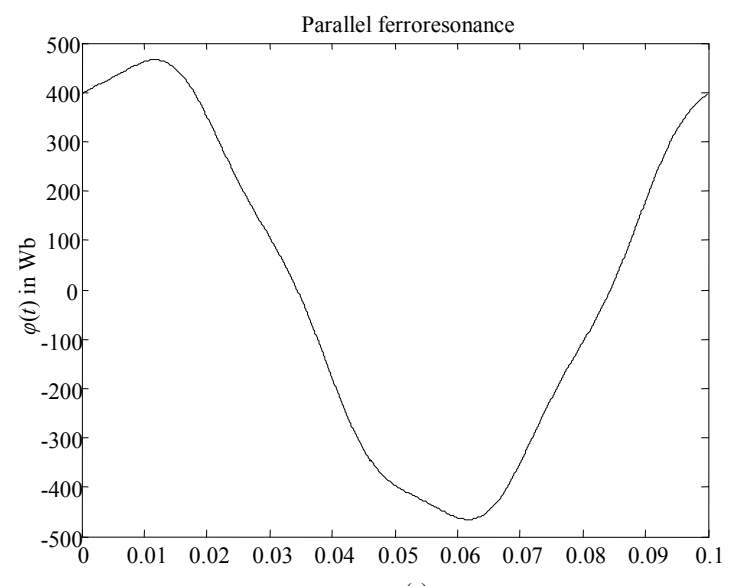

$t(\mathrm{~s})$ voltage $V(t)$ decrease when the row of subharmonic increases. These values are always lower than those reached by the fundamental ferroresonance mode.

- For the same excitation voltage $E$, different subharmonic solutions can exist. One or the other of the solutions appears according to the value of the initial conditions (initial charge on capacitor, remanent flux in the core of the target transformer, switching instant, etc.).

This methodology permits to treat effectively the initialization problems encountered in the Galerkin method and the continuation methods.

\subsubsection{Search for Fractional Subharmonics Modes}

In 1982, EDF recorded a ferroresonance case during the voltage feedback from hydroelectric generators of Chastang on transformers of nuclear power stations of Chinon [5]. Abnormal oscillations at a preponderant frequency of $83.33 \mathrm{~Hz}$ are observed. This frequency corresponds to a period $\frac{3 T_{0}}{5}$ (with $T_{0}=20 \mathrm{~ms}$ is the source period).

We apply the proposed analytical method to simulate this mode type, solution of the circuit of Figure 2 modeling the voltage feedback.

We consider two flux components $\phi_{1}$ and $\phi_{5 / 3}(h=5 / 3$ is not a simple unit fraction) and we adopt the following values: $L_{g}=2.25 \mathrm{H}, R_{g}=20 \Omega, C_{t}=4.5 \mu \mathrm{F}, R_{t}=0.1 \mathrm{M} \Omega$, $L_{d}=0.1 \mathrm{H}$ and $R_{d}=7 \Omega$, which correspond to a line of nominal voltage $225 \mathrm{kV}$ of $370 \mathrm{~km}$ and give a network natural frequency of

$$
f_{r}=\frac{1}{2 \pi \sqrt{L_{g} \cdot C_{t}}}=50 \mathrm{~Hz}
$$

The Galerkin Equations system of is:

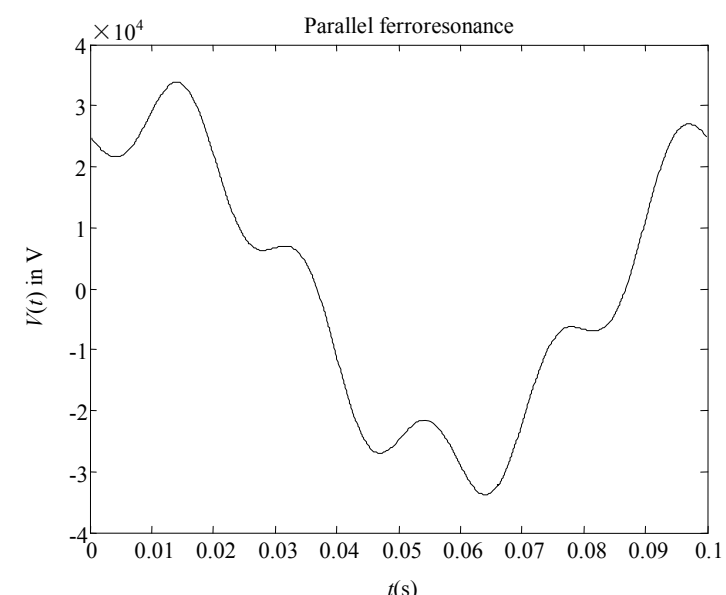

$t(\mathrm{~s})$

Figure 6. SH5 solution: Flux $\varphi(t)$ and voltage $V(t)$ waveforms on the target transformer during one period for the subharmonic-5 ferroresonance for $E=183 \mathrm{kV}$. 

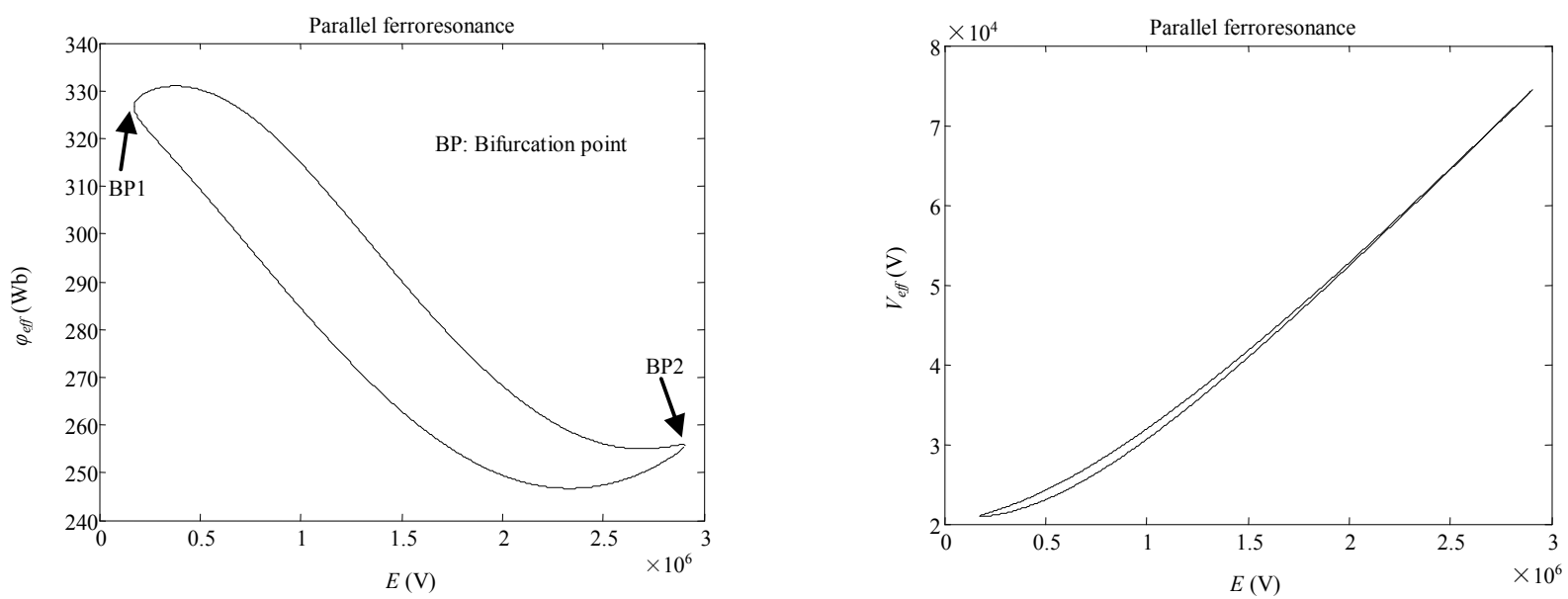

(a)
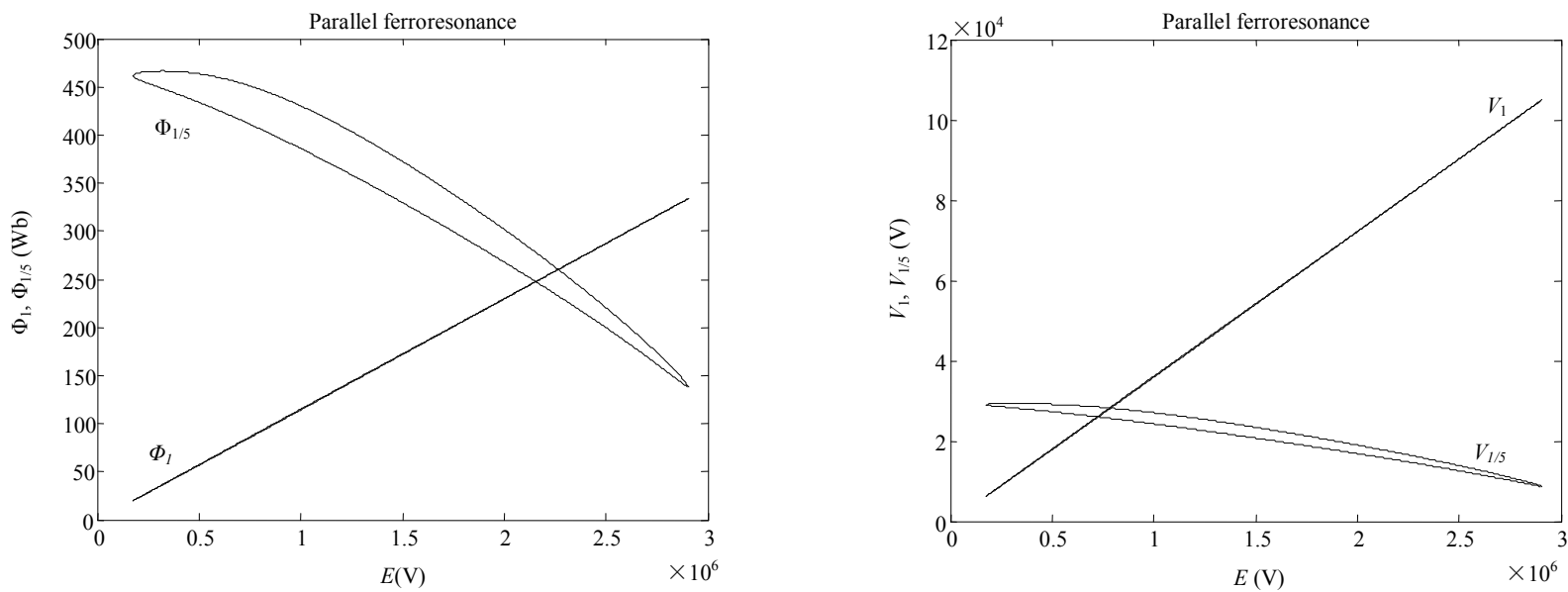

(b)

Figure 7. Bifurcation diagrams of SH5 mode as a function of $E$, for the circuit parameters: $L_{g}=2.25 \mathrm{H}, L_{d}=0.1 \mathrm{H}, C_{t}=129 \mu \mathrm{F}$, $R_{t}=0.1 \mathrm{M} \Omega, R_{g}=1 \Omega$ and $R_{d}=0.5 \Omega$. (a) Existence zones of the SH5 mode versus applied voltage $E$. (b) Continuation of the amplitudes of $\mathrm{SH} 5$ component and the $50 \mathrm{~Hz}$ component.
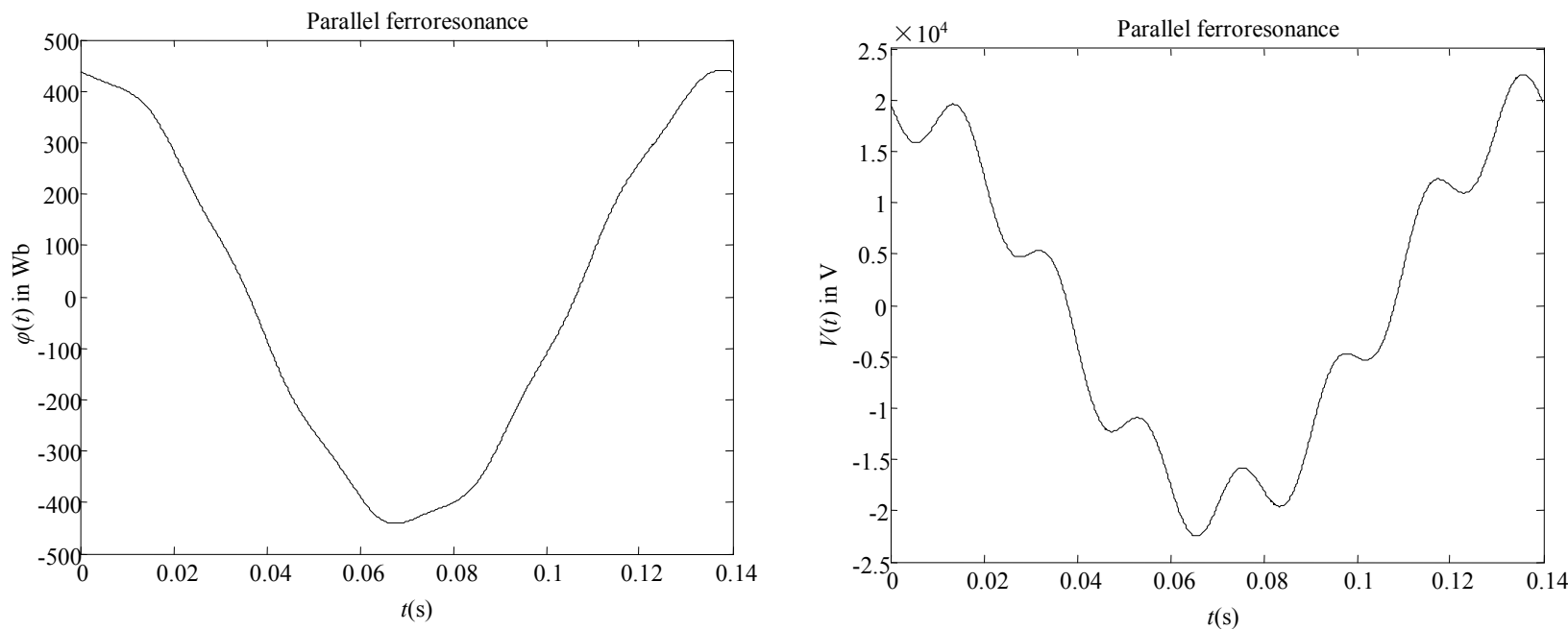

Figure 8. SH7 solution: Flux $\varphi(t)$ and voltage $V(t)$ waveforms on the target transformer during one period for the subharmonic-7 ferroresonance for $E=183 \mathrm{kV}$. 

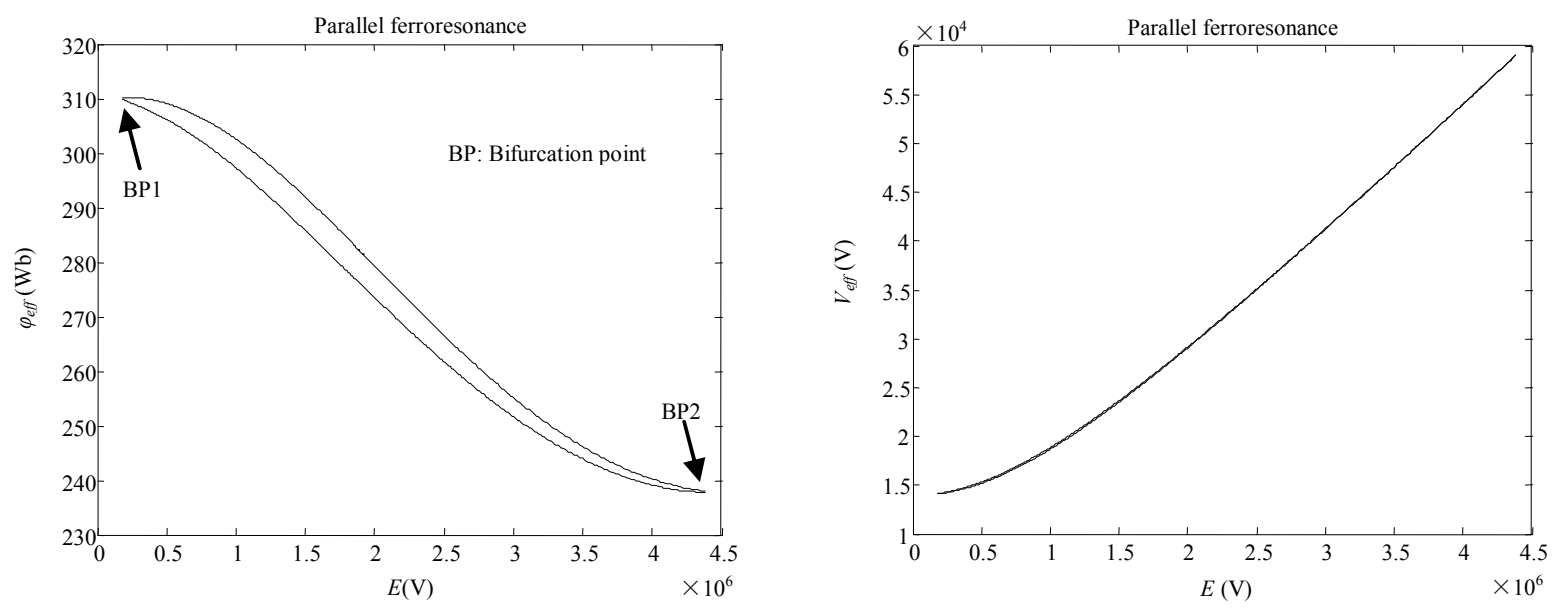

(a)
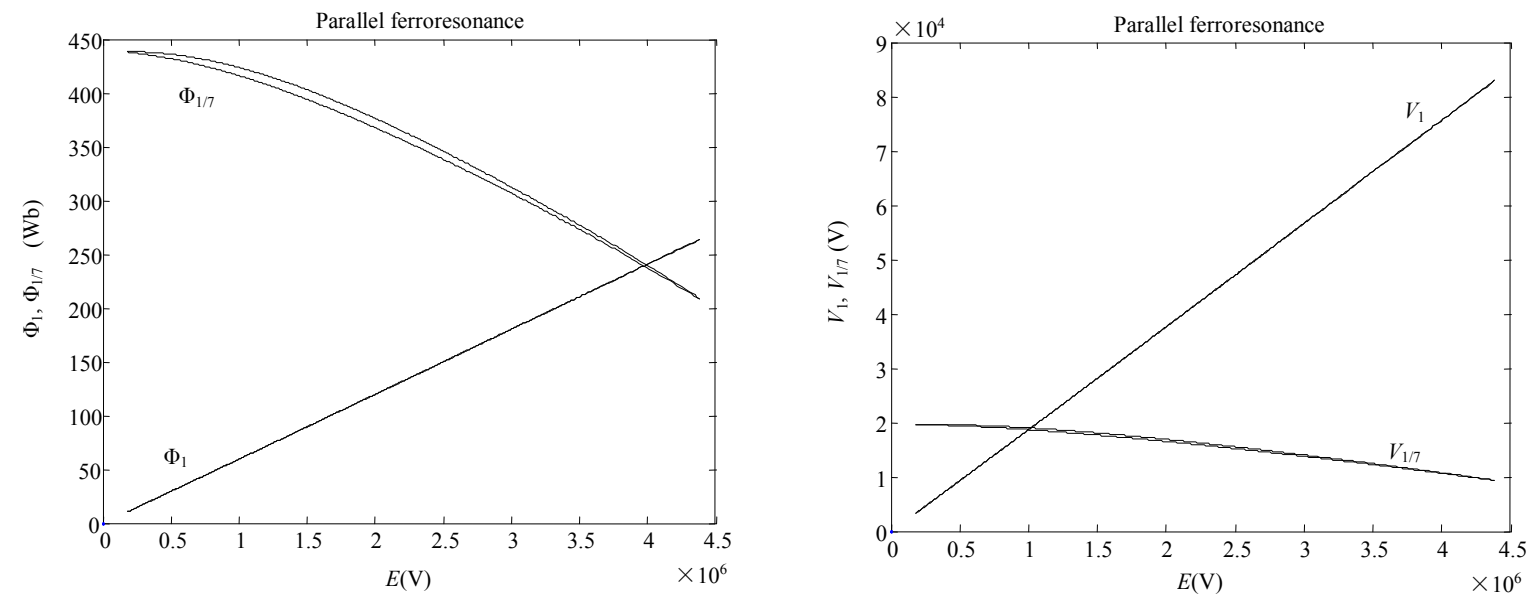

(b)

Figure 9. Bifurcation diagrams of $\mathrm{SH} 7$ mode as a function of $E$, for the circuit parameters: $L_{g}=2.25 \mathrm{H}, L_{d}=0.1 \mathrm{H}, C_{t}=242 \mu \mathrm{F}, R_{t}$ $=1 \mathrm{M} \Omega, R_{g}=0.1 \Omega$ and $R_{d}=0.1 \Omega$. (a) Existence zones of the SH7 mode versus applied voltage $E$. (b) Continuation of the amplitudes of $\mathrm{SH} 7$ component and the $50 \mathrm{~Hz}$ component.
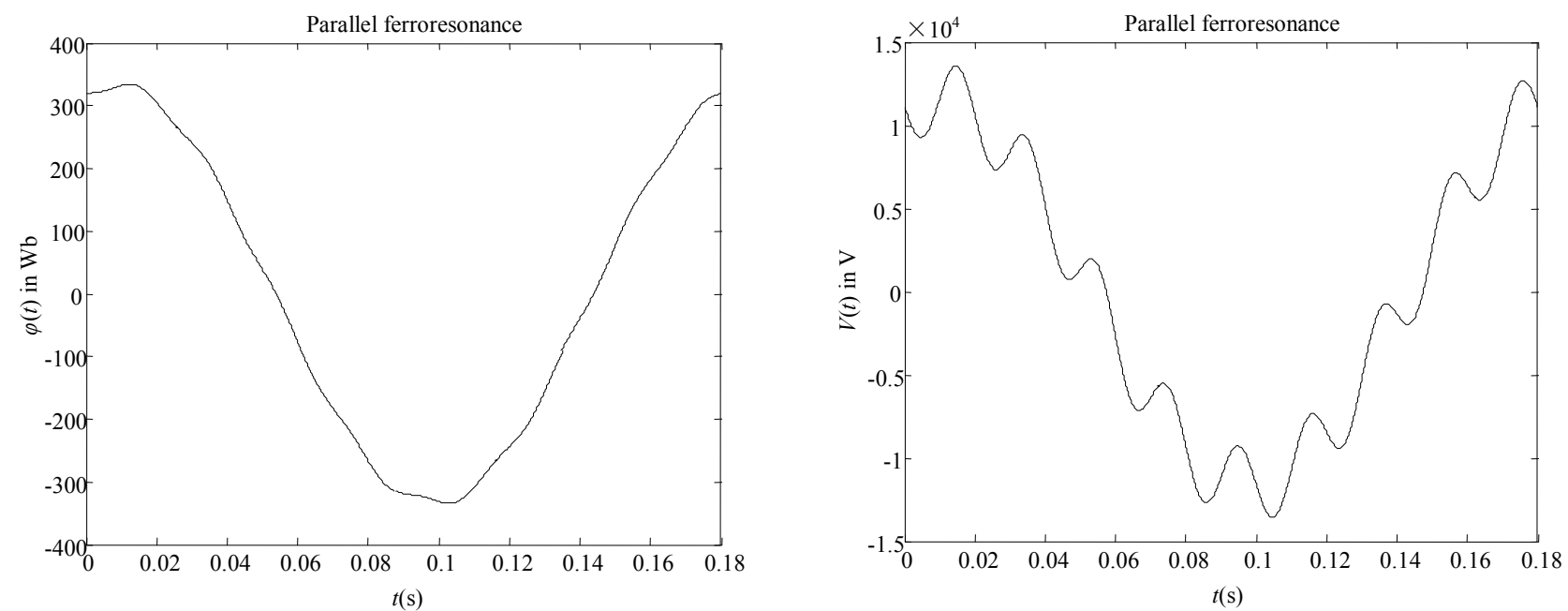

Figure 10. SH9 solution: Flux $\varphi(t)$ and voltage $V(t)$ waveforms on the target transformer during one period for the subharmonic-9 ferroresonance for $E=183 \mathrm{kV}$. 

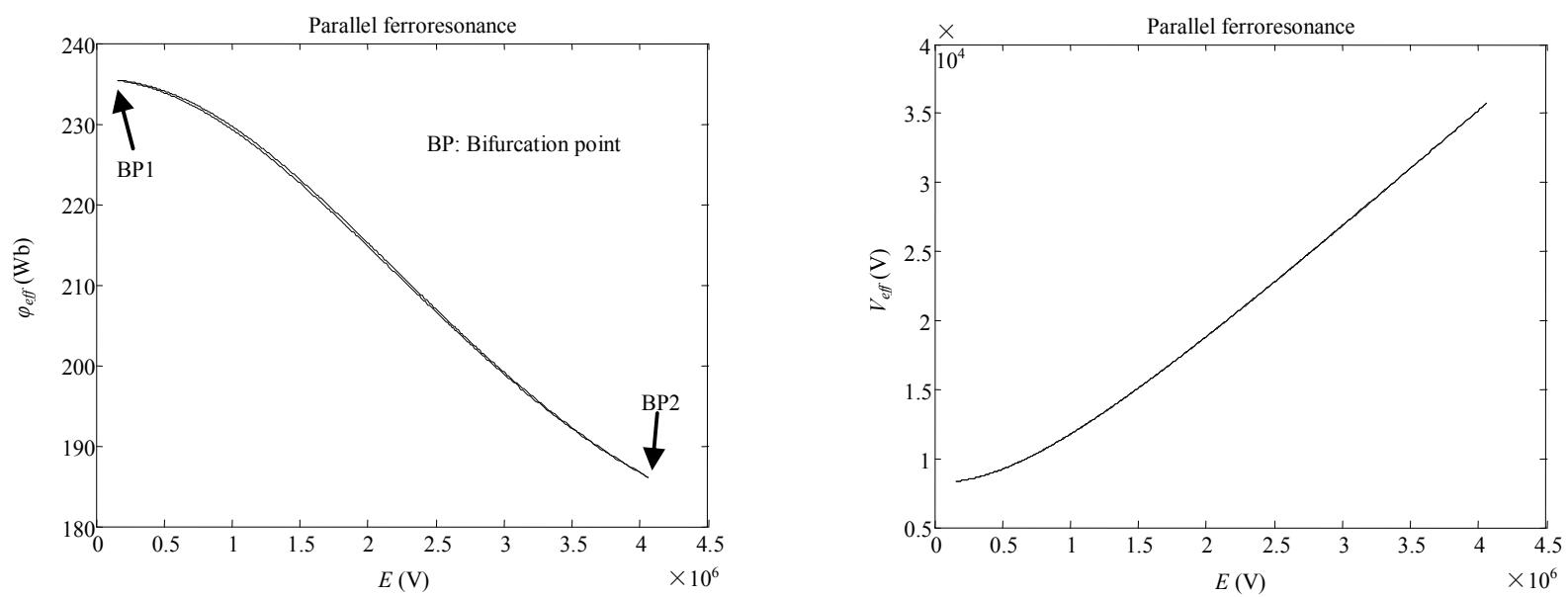

(a)
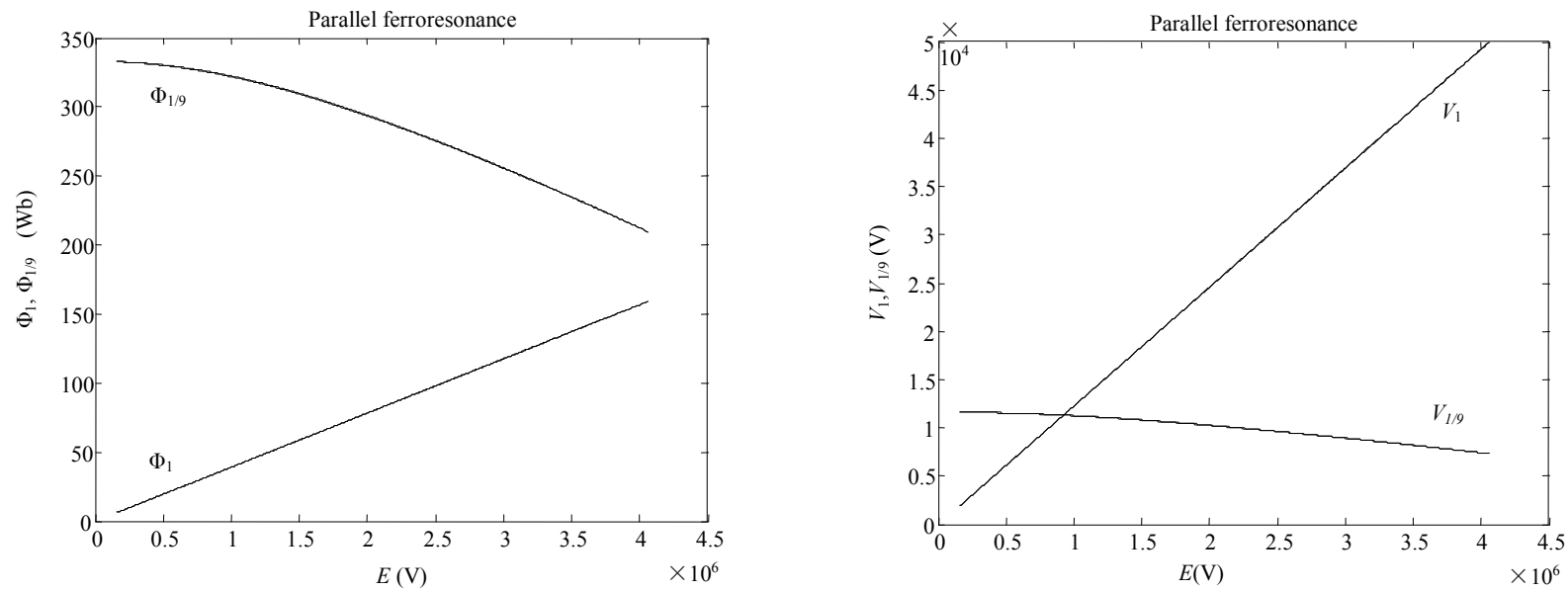

(b)

Figure 11. Bifurcation diagrams of SH9 mode as a function of $E$, for the circuit parameters: $L_{g}=2.25 \mathrm{H}, L_{d}=0.1 \mathrm{H}, C_{t}=370$ $\mu \mathrm{F}, R_{t}=100 \mathrm{M} \Omega, R_{g}=1 \mathrm{~m} \Omega$ and $R_{d}=0 \Omega$. (a) Existence zones of the SH9 mode versus applied voltage $E$. (b) Continuation of the amplitudes of $\mathrm{SH} 9$ component and the $50 \mathrm{~Hz}$ component.
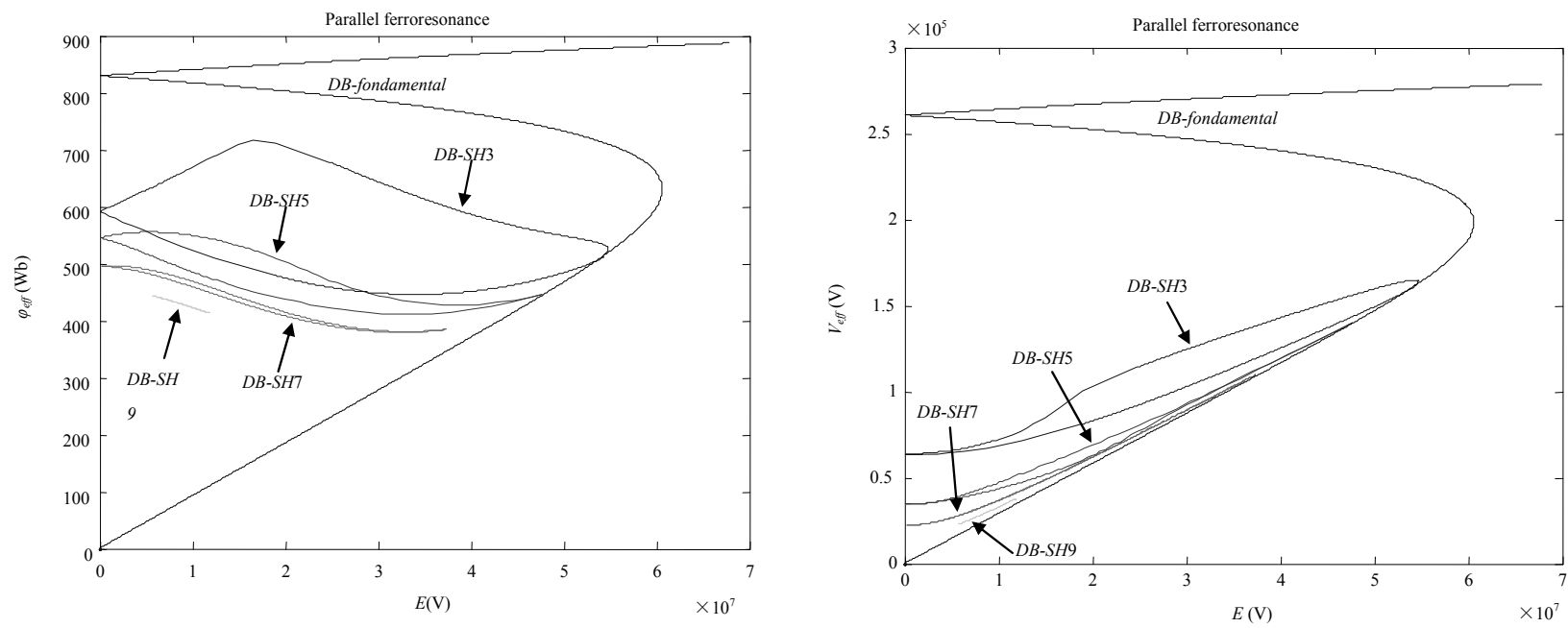

Figure 12. Comparison between bifurcation diagrams of SH3, 5, 7, 9 modes and of fundamental mode for the circuit parameters: $L_{g}=2.25 \mathrm{H}, L_{d}=0 \mathrm{H}, C_{t}=1100 \mu \mathrm{F}, R_{t}=0.1 \mathrm{M} \Omega, R_{g}=1 \Omega$ and $R_{d}=0 \Omega$. 


$$
\begin{aligned}
& \frac{5 \omega}{3} \phi_{\frac{5}{3} c}-R_{\frac{5}{3}} I_{\frac{5}{3} s}+X_{\frac{5}{3} \frac{5}{3} c}=0 \\
& \frac{5 \omega}{3} \phi_{\frac{5}{3} s}+R_{\frac{5}{3}} I_{\frac{5}{3} c}+X_{\frac{5}{3}} I_{\frac{5}{3} s}=0 \\
& \omega \phi_{1 c}-R_{1} I_{1 s}+X_{1} I_{1 c}=-E_{1 s} \\
& \omega \phi_{1 s}+R_{1} I_{1 c}+X_{1} I_{1 s}=E_{1 c}
\end{aligned}
$$

We obtain a subharmonic 3 mode (Figure 13) whose harmonic 5 of frequency $f=\frac{5}{3} f_{0}=83.33 \mathrm{~Hz}$ (with $f_{0}=\frac{1}{T_{0}}=50 \mathrm{~Hz}$ is the source frequency) is preponderant in its spectrum. It is the synchronization phenomenon between the natural period of the free system $T=\frac{3 T_{0}}{5}$ without excitation or losses (called Hamiltonian system) and the source period $T_{0}$, which gives us a fractional subharmonic mode $\mathrm{SH} 3 / 5$ oscillating at a common period which is here $3 T_{0}$. We announce that for the ordinary ferroresonant circuits, the maximum period of free oscillation is about $100 \mathrm{~ms}$ at a few seconds and the minimal period is about of the millisecond.

The continuation of this mode in function of the excitation $E$ is summarized by the bifurcation diagrams of Figure 14: it is an isolat, result awaited for the subharmonics modes. These diagrams show that, for the network parameters values, ferroresonance may occur for nominal phase-to-neutral network voltage $130 \mathrm{kV}$ (whether the possible initial condition values are responsible for initiating ferroresonance).

\subsubsection{Search for Harmonics Modes}

We apply the method previously described for systematic calculation of the periodic modes, of the same excitation

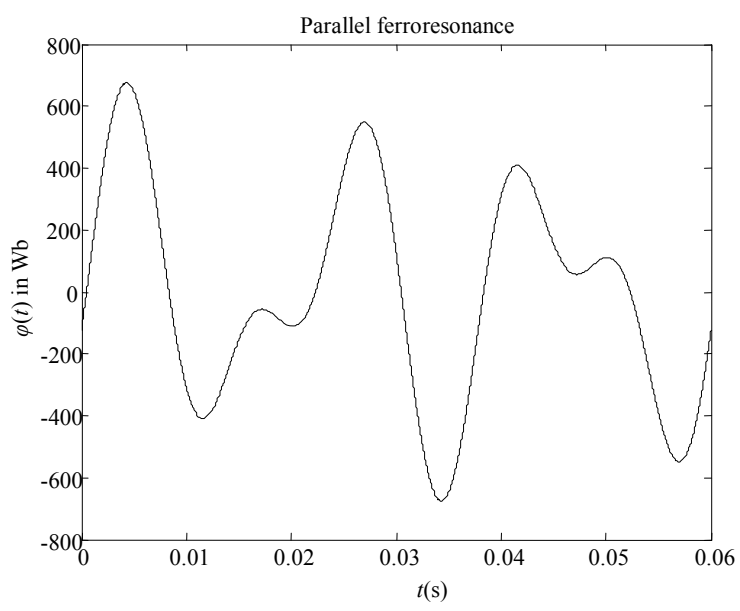

period, rich in harmonic $h$. In this case, flux is described by two harmonic components $\phi_{1}$ and $\phi_{h}$ with $h$ positive integer. We speak about the harmonic $h$ mode (we use the notation $\mathrm{H} h$ ) when the amplitude $\phi_{h}$ becomes important and superior to $\phi_{1}$.

We study, in this paragraph, the harmonic 3, 5 and 7 modes, for real situations of Figure 2. Setting of Equations is similar to that adopted for the subharmonics modes.

Figure 15 shows, for $E_{\text {nom }}=183 \mathrm{kV}$, the waveforms of flux $\varphi(t)$ and voltage $V(t)$ at the transformer terminals in search case for a H3 mode. By examining these curves, we note that each one contains three similar maximums over a source period; that means that the solution obtained is very rich in harmonic $3(f=150 \mathrm{~Hz})$. The continuation of this mode, in function of the excitation voltage $E$ begins with the null trivial solution $(\varphi=0, V=0)$ for $E=0$ (Figure 16). When $E$ increases, one meets two bifurcations of the limit point type $B P_{1}$ and $B P_{2}$ specifying the true zone of existence of the $\mathrm{H} 3$ mode. In fact, inside this zone where the tension $E$ is understood between $152 \mathrm{kV}$ and $240 \mathrm{kV}$, the amplitude of the third-harmonic component $\left(V_{3}\right)$ is important in front of the fundamental component $50 \mathrm{~Hz}\left(V_{1}\right)$ (Figure 16(b)) and the corresponding solutions are characterized by dangerous overvoltages (the nominal amplitude of the network voltage is $183 \mathrm{kV}$ ).

On Figures 17-20, we summarize the results of the H5 and $\mathrm{H} 7$ modes (they are real cases). Qualitatively, the remarks previously quoted on the DB-H3 are retained for the DB-H5 and DB-H7.

To have an overall view of the results, we compare the bifurcation diagrams of the harmonic 3, 5, 7 modes and the fundamental mode (Figures 21) for the following real case: $L_{g}=2.25 \mathrm{H}, R_{g}=20 \Omega, C_{t}=1 \mu \mathrm{F}, R_{t}=0.1 \mathrm{M} \Omega$, $L_{d}=0.1 \mathrm{H}$ and $R_{d}=7 \Omega$. Then we conclude that:

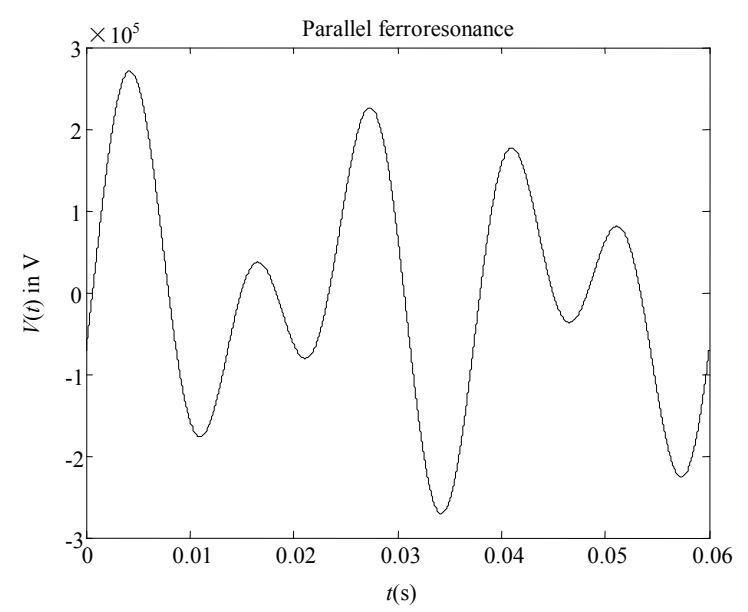

Figure 13. SH3/5 solution: Flux $\varphi(t)$ and voltage $V(t)$ waveforms on the target transformer during one period for the fractional subharmonic-3/5 ferroresonance for $E=183 \mathrm{kV}$. 

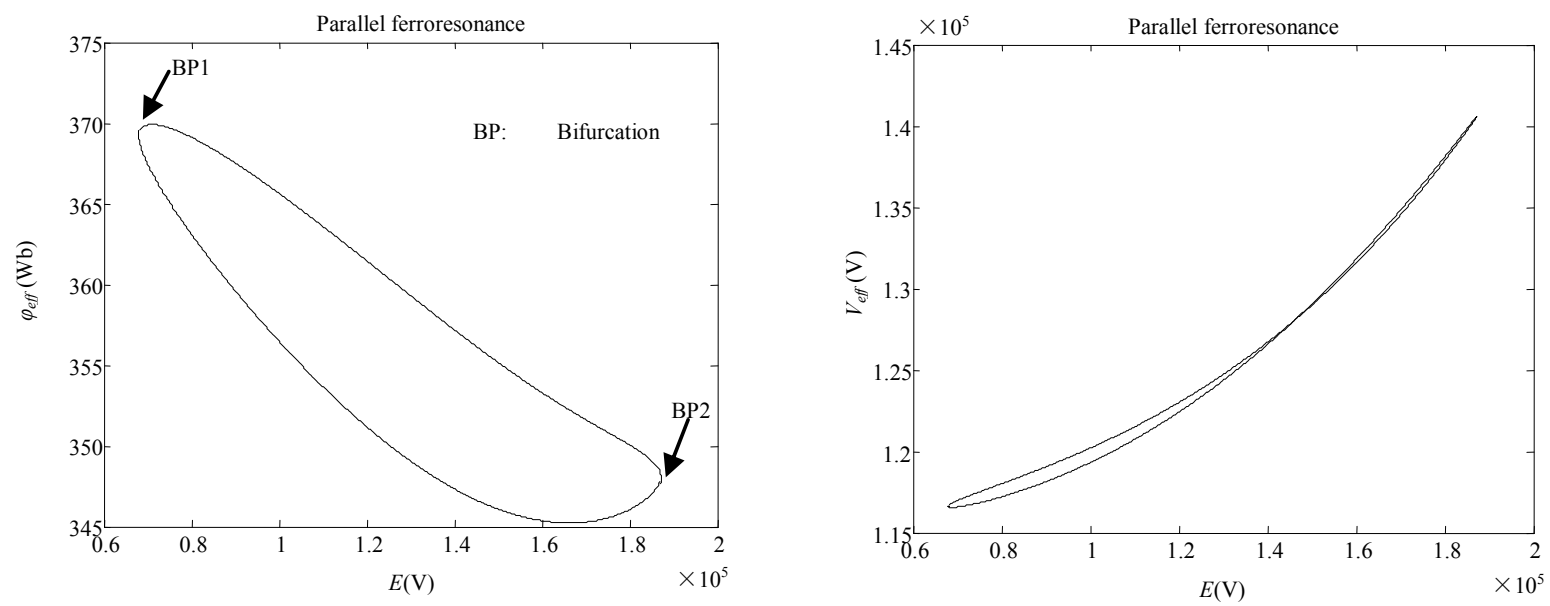

(a)
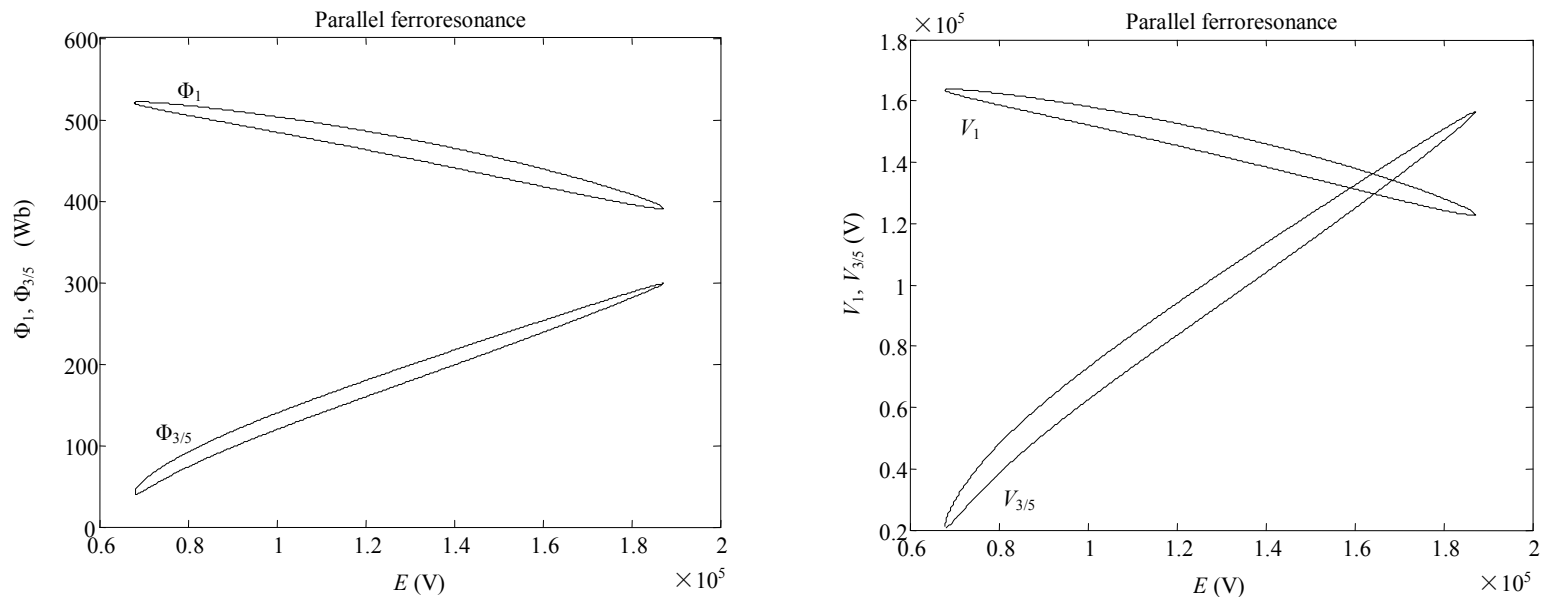

(b)

Figure 14. Bifurcation diagrams of $\mathrm{SH} 3 / 5$ mode as a function of $E$, for the circuit parameters: $L_{g}=2.25 \mathrm{H}, L_{d}=0.1 \mathrm{H}, C_{t}=4.5$ $\mu \mathrm{F}, R_{t}=0.1 \mathrm{M} \Omega, R_{g}=20 \Omega$ and $R_{d}=7 \Omega$. (a) Existence zones of the SH3/5 mode versus applied voltage $E$. (b) Continuation of the amplitudes of $\mathrm{SH} 3 / 5$ component and the $50 \mathrm{~Hz}$ component.
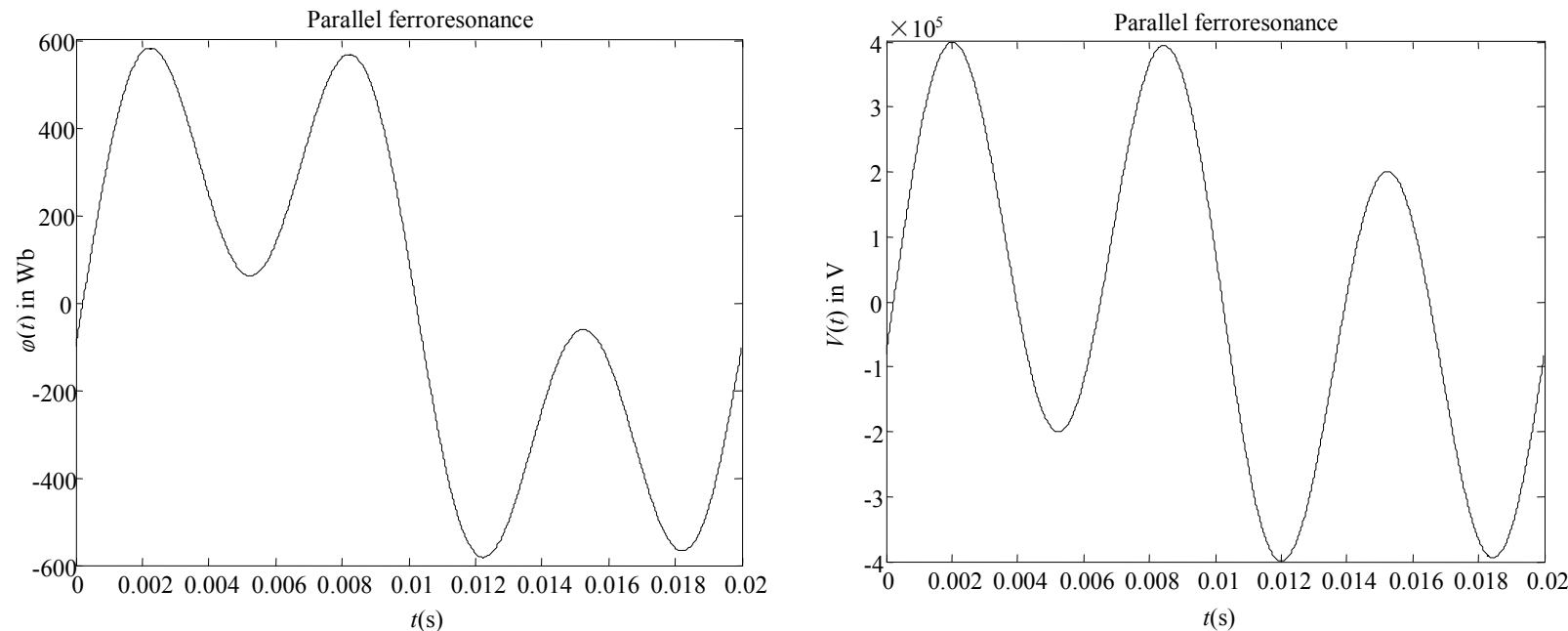

Figure 15. H3 solution: Flux $\varphi(t)$ and voltage $V(t)$ waveforms on the target transformer during one period for the harmonic-3 ferroresonance for $E=183 \mathrm{kV}$. 

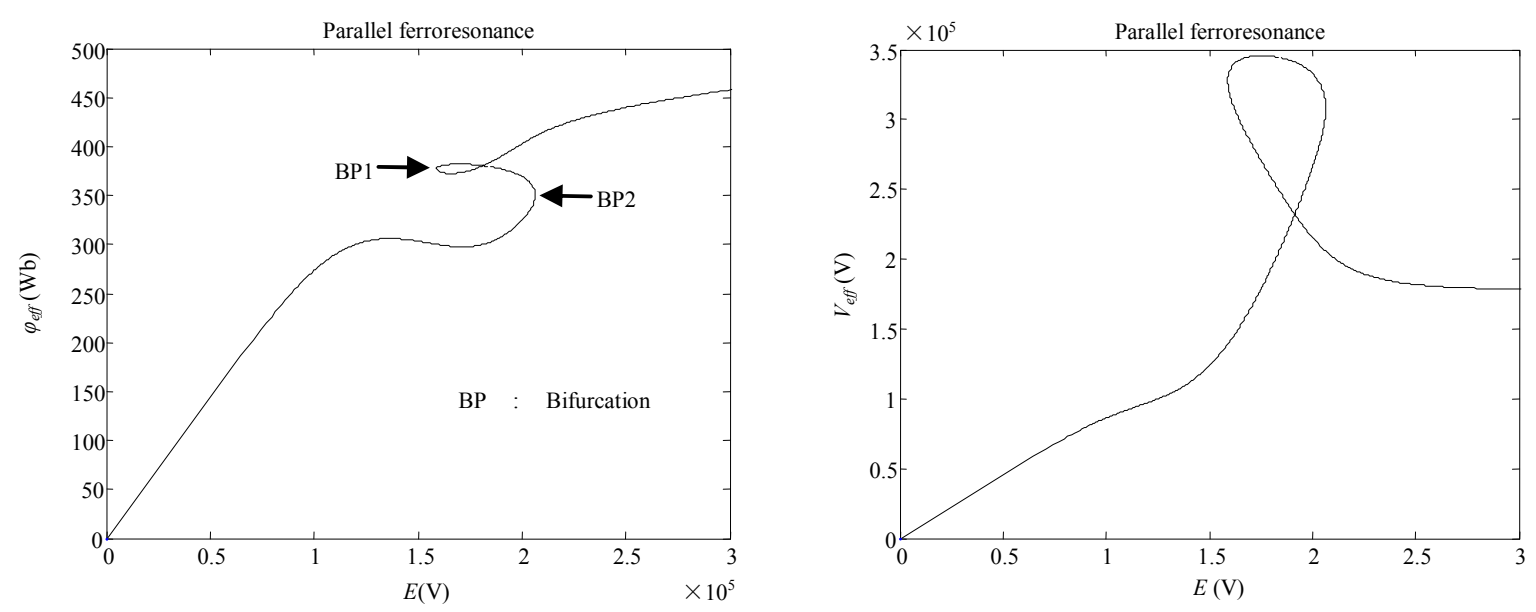

(a)
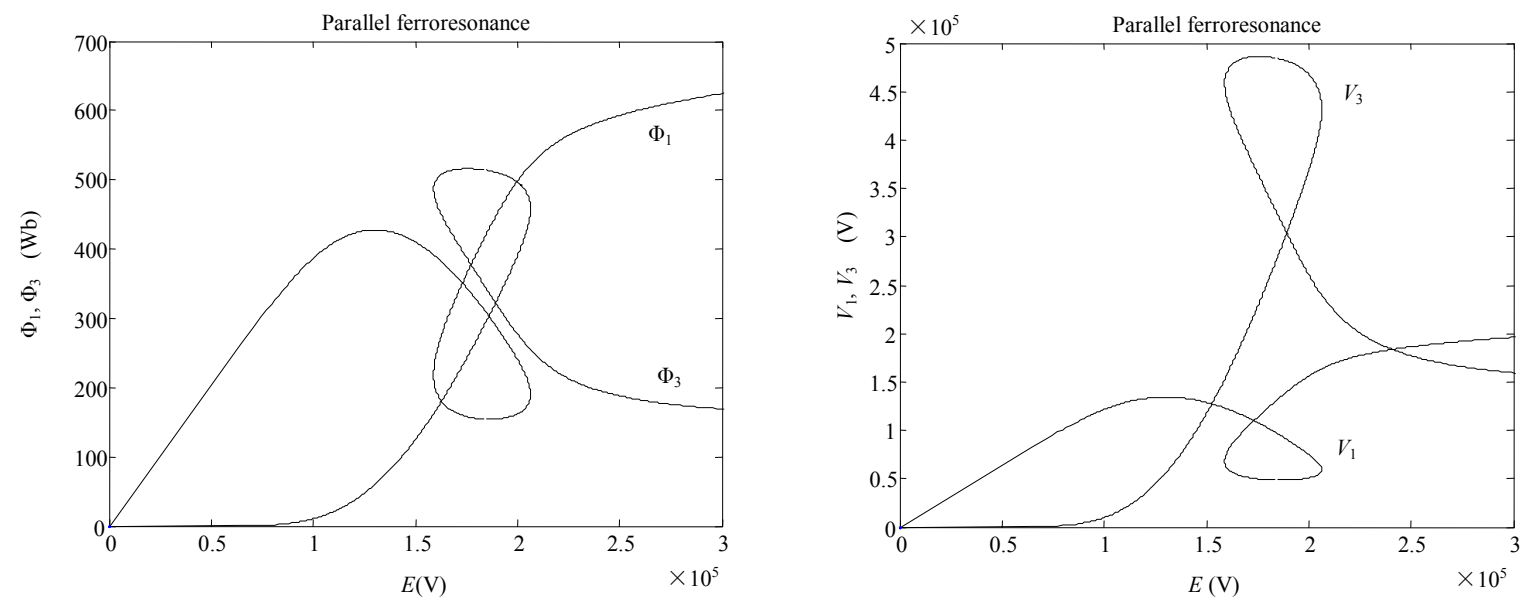

(b)

Figure 16. Bifurcation diagrams of $\mathrm{H3}$ mode as a function of $E$, for the circuit parameters: $L_{g}=2.25 \mathrm{H}, L_{d}=0.1 \mathrm{H}, C_{t}=1 \mu \mathrm{F}$, $R_{t}=0.1 \mathrm{M} \Omega, R_{g}=20 \Omega$ and $R_{d}=7 \Omega$. (a) Existence zones of the $\mathrm{H3}$ mode versus applied voltage $E$. (b) Continuation of the amplitudes of $\mathrm{H3}$ component and the $50 \mathrm{~Hz}$ component.

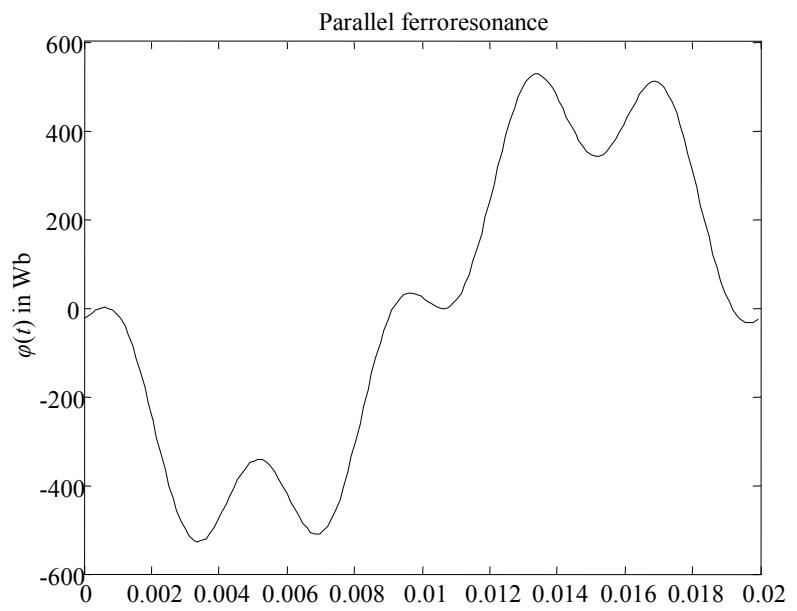

$t(\mathrm{~s})$

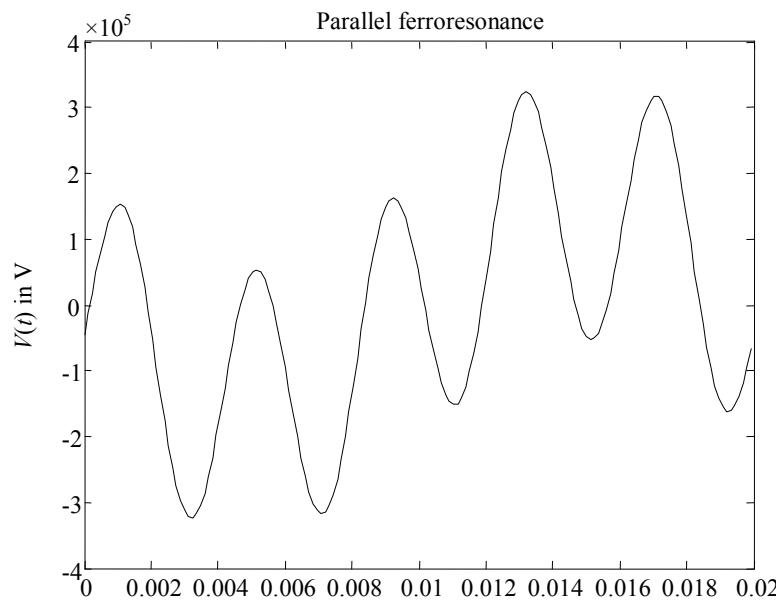

$t(\mathrm{~s})$

Figure 17. H5 solution: Flux $\varphi(t)$ and voltage $V(t)$ waveforms on the target transformer during one period for the harmonic-5 ferroresonance for $E=183 \mathrm{kV}$. 

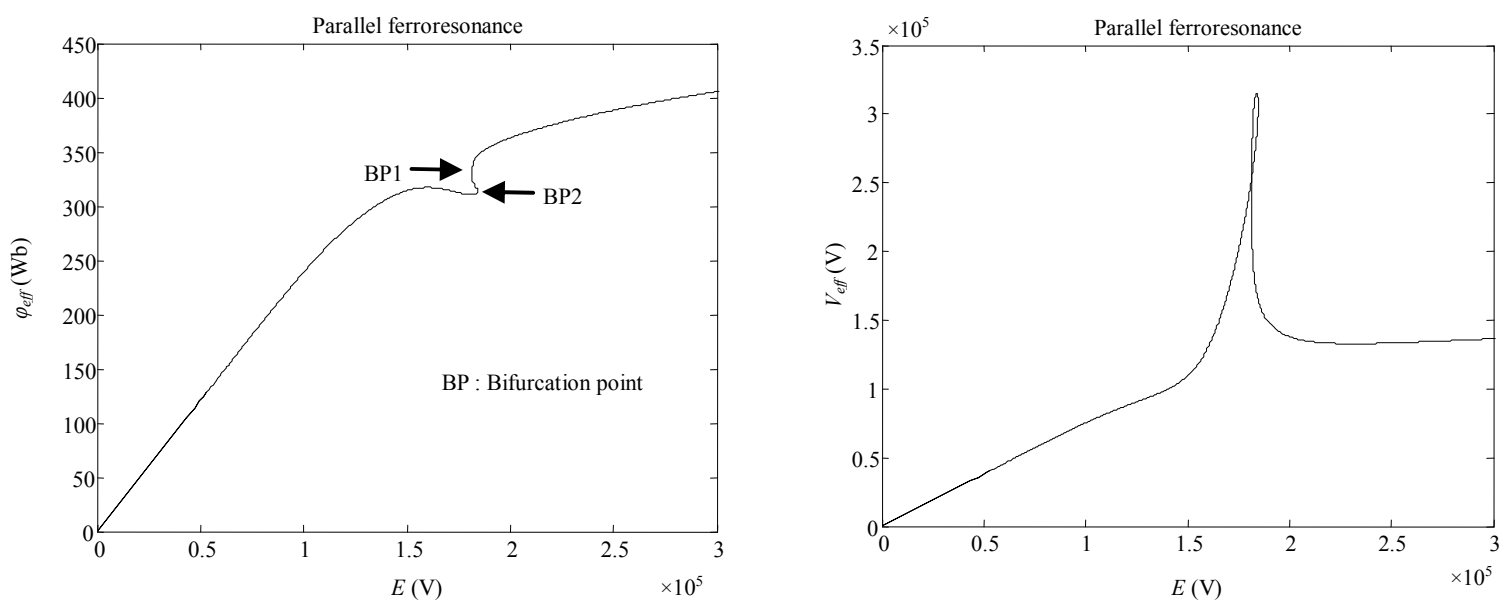

(a)
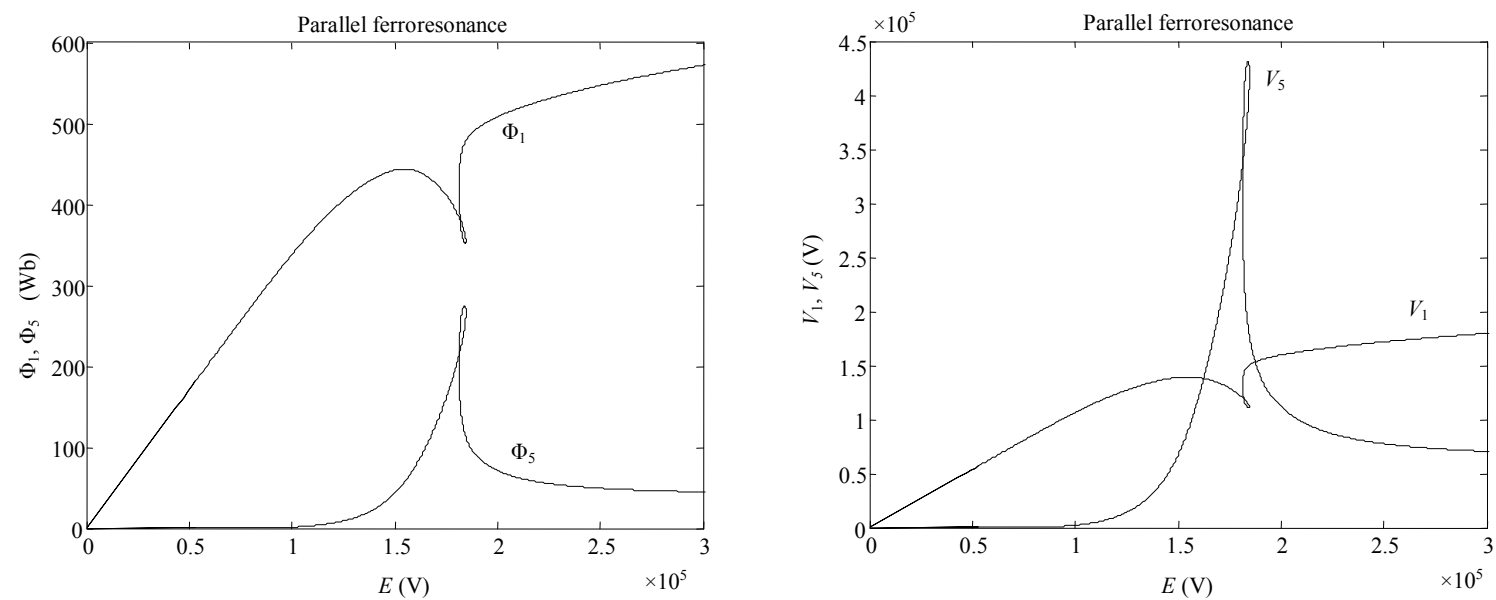

(b)

Figure 18. Bifurcation diagrams of $\mathrm{H5}$ mode as a function of $E$, for the circuit parameters: $L_{g}=2.25 \mathrm{H}, L_{d}=0.1 \mathrm{H}, C_{t}=0.333$ $\mu \mathrm{F}, R_{t}=0.1 \mathrm{M} \Omega, R_{g}=20 \Omega$ and $R_{d}=7 \Omega$. (a) Existence zones of the $\mathrm{H5}$ mode versus applied voltage $E$. (b) Continuation of the amplitudes of $\mathrm{H} 5$ component and the $50 \mathrm{~Hz}$ component.
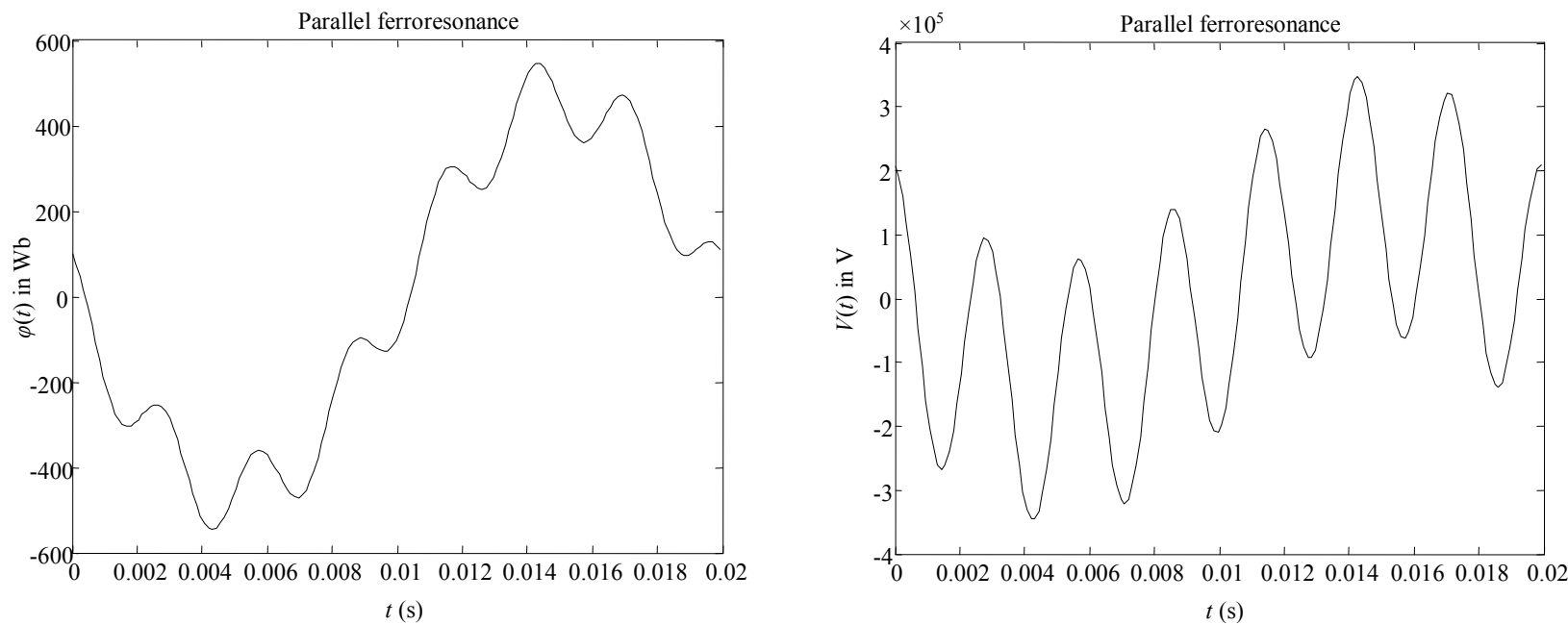

Figure 19. H7 solution: Flux $\varphi(t)$ and voltage $V(t)$ waveforms on the target transformer uring one period for the harmonic-7 ferroresonance for $E=183 \mathrm{kV}$. 

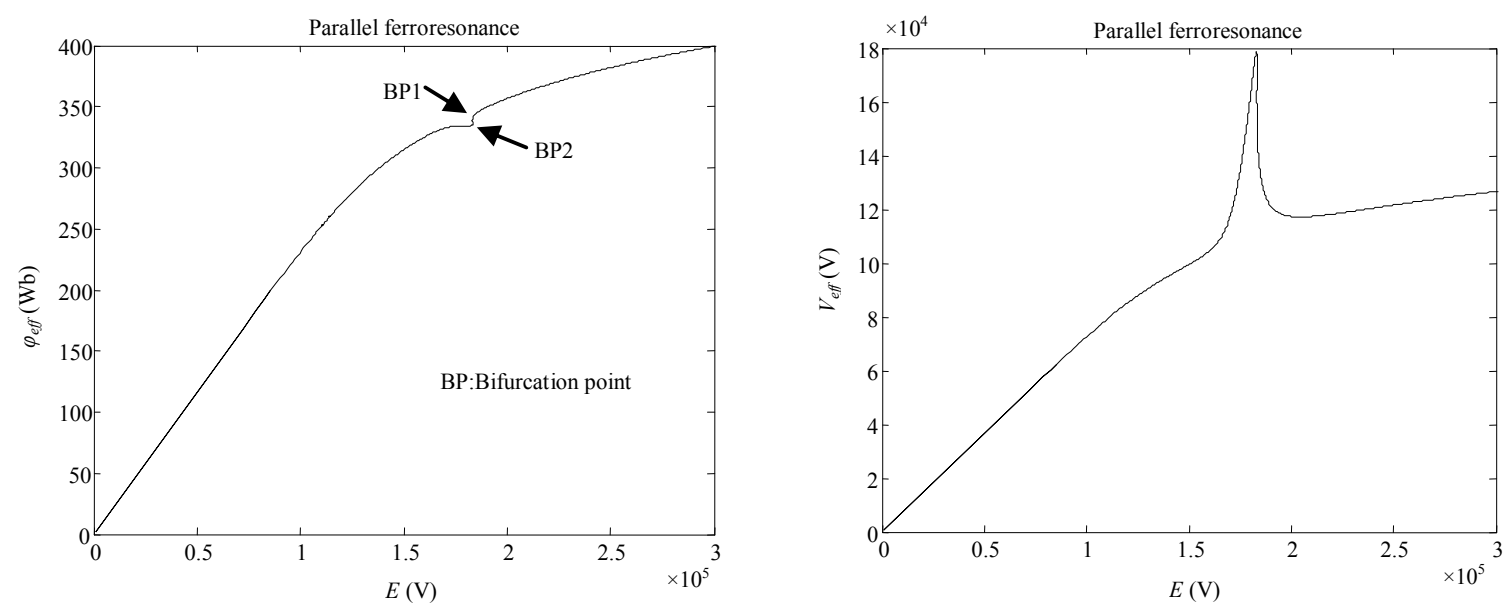

(a)
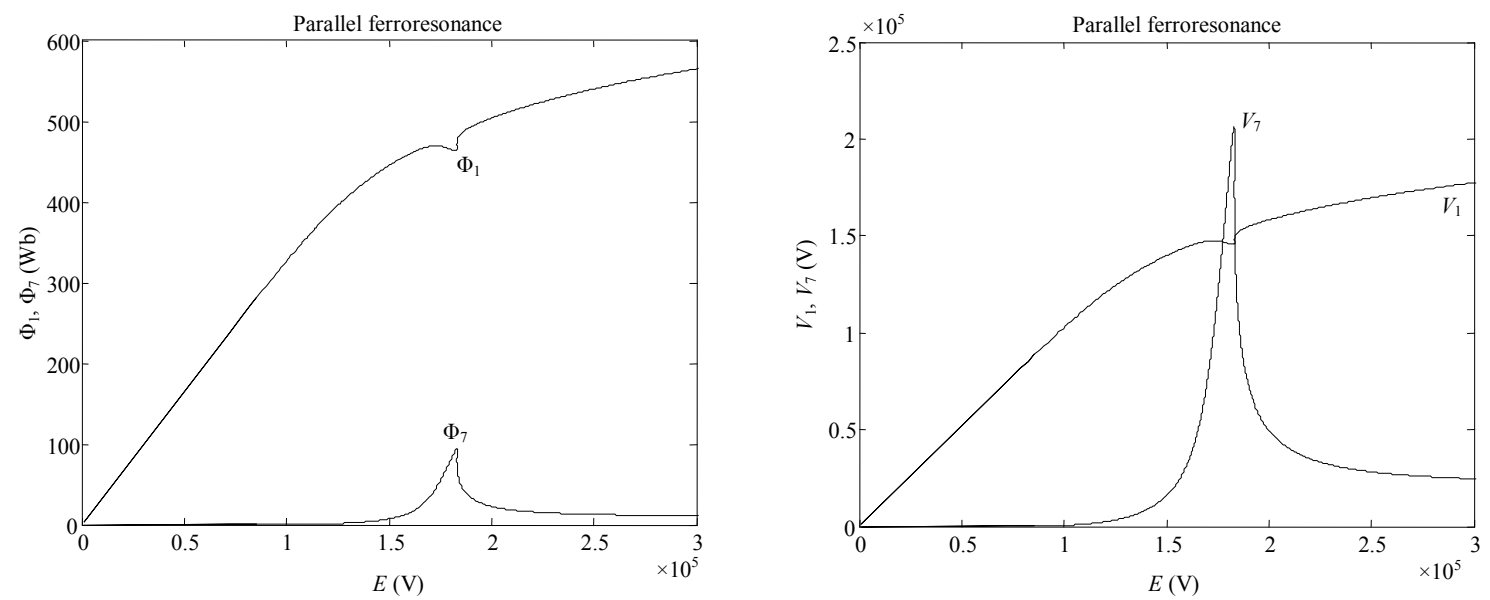

(b)

Figure 20. Bifurcation diagrams of $\mathrm{H} 7 \mathrm{mode}$ as a function of $E$, for the circuit parameters: $L_{g}=2.25 \mathrm{H}, L_{d}=0.1 \mathrm{H}, C_{t}=0.178$ $\mu \mathrm{F}, R_{t}=0.1 \mathrm{M} \Omega, R_{g}=20 \Omega$ and $R_{d}=7 \Omega$. (a) Existence zones of the $\mathrm{H} 7$ mode versus applied voltage $E$. (b) Continuation of the amplitudes of $\mathrm{H} 7$ component and the $50 \mathrm{~Hz}$ component.
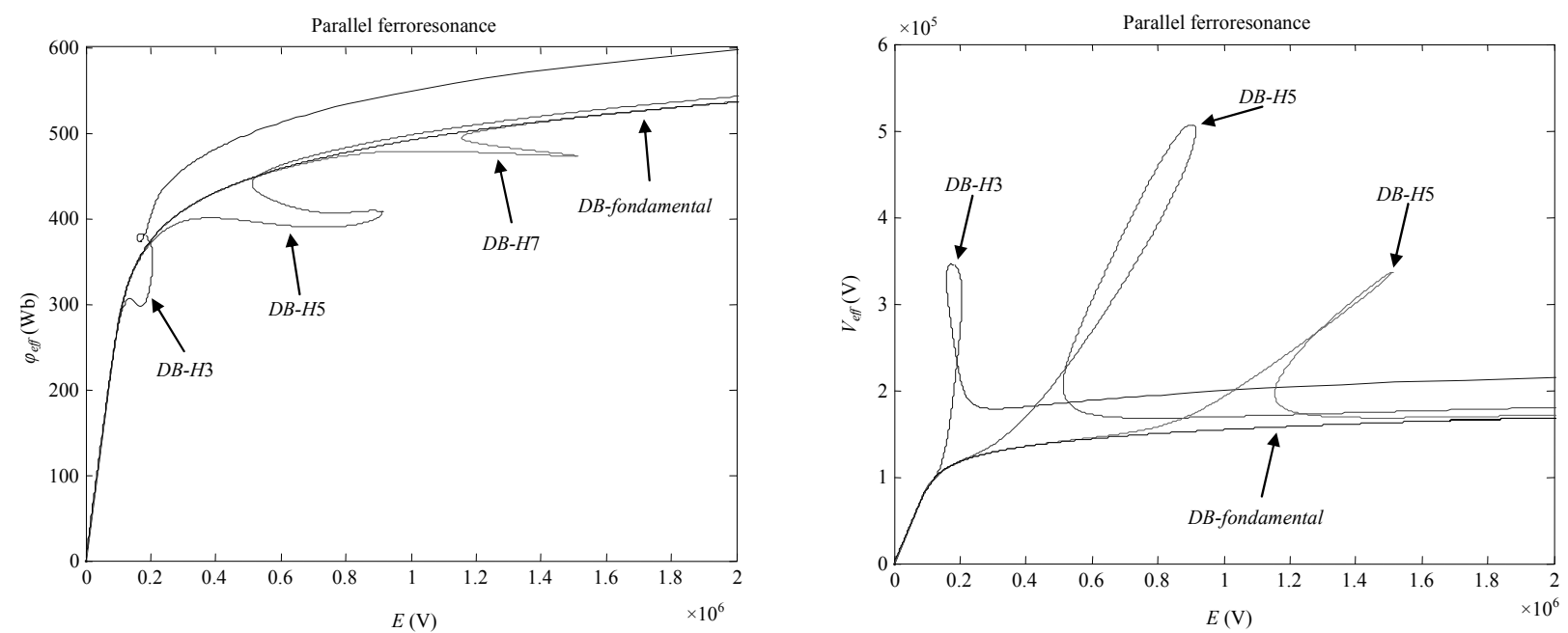

Figure 21. Comparison between bifurcation diagrams of $\mathrm{H3}, 5,7$ modes and of fundamental mode for the circuit parameters: $L_{g}=2.25 \mathrm{H}, L_{d}=0.1 \mathrm{H}, C_{t}=1 \mu \mathrm{F}, R_{t}=0.1 \mathrm{M} \Omega, R_{g}=20 \Omega$ and $R_{d}=7 \Omega$. 
- The bifurcation diagrams of the harmonic modes are complex curves always starting with the null trivial solution.

- The existence domains of these oscillation modes and their voltage levels $E$ increase when the row $h$ of harmonic increases. The existence minimum voltages are much become large as the row $h$ is large. For $h>3$, they are clearly higher than the nominal voltage.

- The voltage domain $E$ situated between the existence superior limit of $\mathrm{H} 3$ mode and the existence inferior limit of the $\mathrm{H} 5$ mode is probably rich of the four-harmonic component (it is the H4 mode).

- The source voltage applied is then determining final mode: in fact, while varying in a monotonous way the excitation voltage $E$, we observe in the increasing order the appearance of the various harmonic modes (even and odd).

- The maximum rms values reached by the voltage $V(t)$ (overvoltages) are always superior to those reached by the fundamental mode.

\subsection{Study of Series Ferroresonance}

We apply this method to computation of the periodic ferroresonant modes of the circuit of Figure 22, which describes correctly the practical problems of the series ferroresonance. Most commonly, this type of situation is achieved when a magnetic voltage transformer (the nonlinear inductance) is connected to busbar separated by the grading capacitance of an open circuit breaker (the series capacitance) [1-4]. This capacitance is the capital element revealing the ferroresonance.

The physical parameters of this circuit are:

$E$ : amplitude of the supply voltage $e(t)=E \cos (100 \pi t)$,

$C$ : equivalent capacitance of the circuit, constituted by all capacitances of the circuit breaker, the transformer and the busbar section,

$l$ : linear inductance of the circuit,

$R_{1}$ and $R_{2}$ : series and parallel losses of the circuit.

The magnetic characteristic is defined by:

$i(\varphi)=10^{-3} \varphi+2,34.10^{-34} \varphi^{9}$. It corresponds to a real voltage transformer, single-phase, of nominal primary

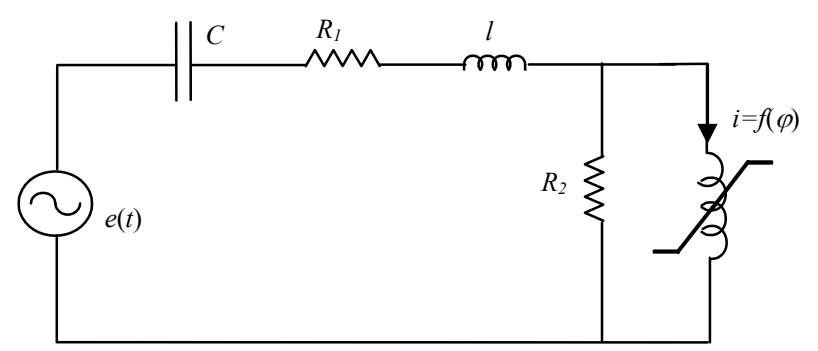

Figure 22. Series, single-phase, nonlinear ferroresonant circuit. voltage $230 \mathrm{kV}$.

We adopt the values $R_{1}=32 \mathrm{k} \Omega ; C=400 \mathrm{pF} ; R_{2}=714$ $\mathrm{M} \Omega$ and $l=0 \mathrm{H}$, which represents a real case allowing the simultaneous study of the various possible ferroresonant modes (fundamental, subharmonics 3, 5, 7, 9 and $3 / 5$ and harmonics 3, 5 and 7).

\subsubsection{Search for Integer Subharmonics Modes}

The row $h$ is a simple fraction of the unit. Figures 23-26 respectively give the initial solutions of the $\mathrm{SH} 3, \mathrm{SH} 5$, SH7 and SH9 modes, obtained for the crest value of the nominal voltage $E_{\text {nоm }}=327 \mathrm{kV}$.

From these initial solutions, we vary step-by-step the continuation parameter $Q$; we obtain the corresponding bifurcation diagrams: they are solution isolats comprising two bifurcation points as in the case of the parallel ferroresonance (Figures 27-30).

Likewise the existence zones of these subharmonics modes are reduced as the parallel losses of the system become large. Indeed, the phenomenon disappears as soon as the losses become important. All this confirms the regulate action that parallel losses have on the various phenomena of the series ferroresonance; their role is much more important than the series losses.

The superposition of the obtained bifurcation diagrams and that of the fundamental mode is given by Figure 31. We find the same conclusions as that of the parallel ferroresonance. The levels of flux $\varphi(t)$ and voltage $V(t)$, for the same circuit parameter decrease when the row of subharmonic increases and they are always lower than those reached by the fundamental ferroresonance mode.

\subsubsection{Search for Fractional Subharmonics Modes}

We apply the method to the situation where $h$ is not a simple unit fraction, by studying the case of the fractional subharmonic mode $\mathrm{SH} 3 / 5$. We obtain a subharmonic 3 mode (Figure 32) whose harmonic $5(83.33 \mathrm{~Hz}$ : which represents the frequency of the phenomenon studied $\mathrm{SH} 3 / 5$ ) is predominating only for unrealistic values of $E$ (Figure 33(b)).

By examining the oscillation waveform of the obtained solution (Figure 32), for $E=E_{\text {nom }}=327 \mathrm{kV}$, we observe that it contains three maximums over a period; that means that this solution is rich in harmonic 3 (50 $\mathrm{Hz})$.

The continuation of this mode as a function of $E$ gives a bifurcation diagram (solutions isolat) comprising 4 singularities $B P_{1}, B P_{2}, B P_{3}$ and $B P_{4}$ (Figure 33(a)). We note that for certain excitation voltage values $E$, four solutions are possible, whereas for others, the system has only two solutions. Obtaining one or another depends on the initial conditions. 

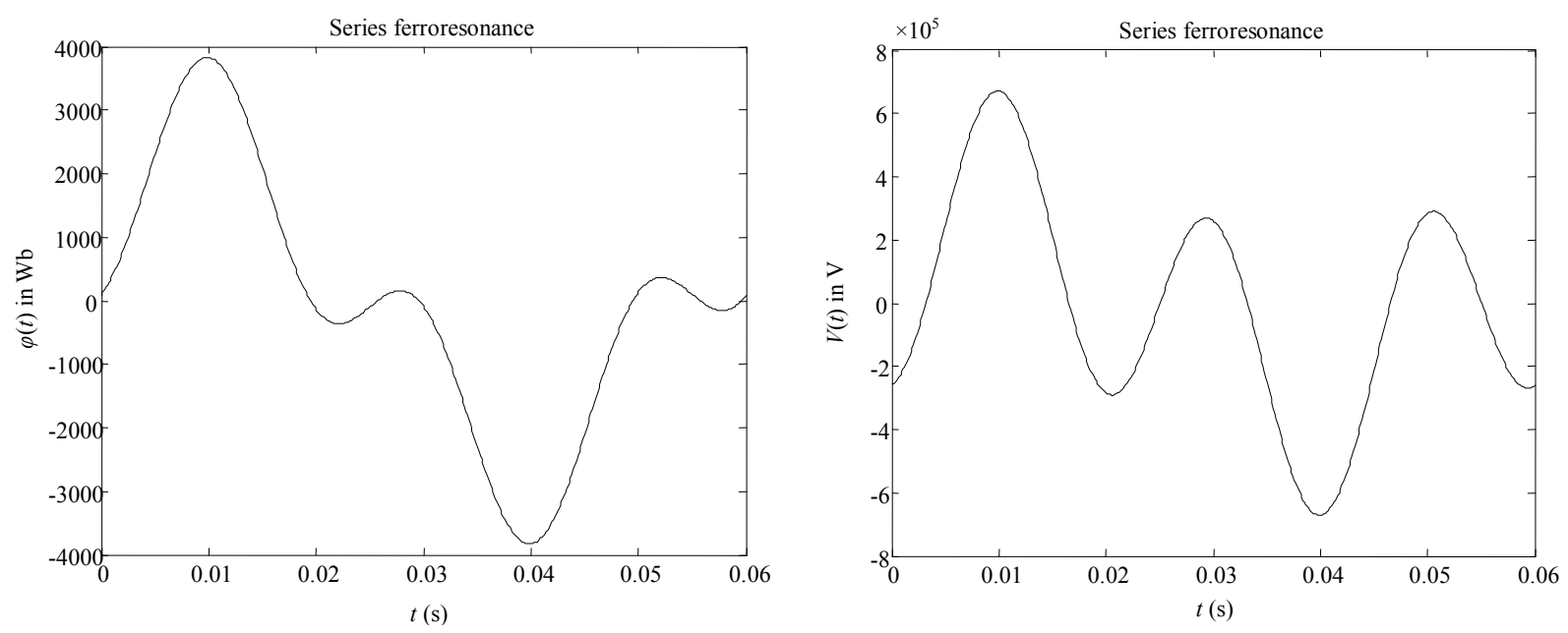

Figure 23. SH3 solution: Flux $\varphi(t)$ and voltage $V(t)$ waveforms on the target transformer during one period for the subharmonic-3 ferroresonance for $E=327 \mathrm{kV}$ and $R_{2}=714 \mathrm{M} \Omega$.
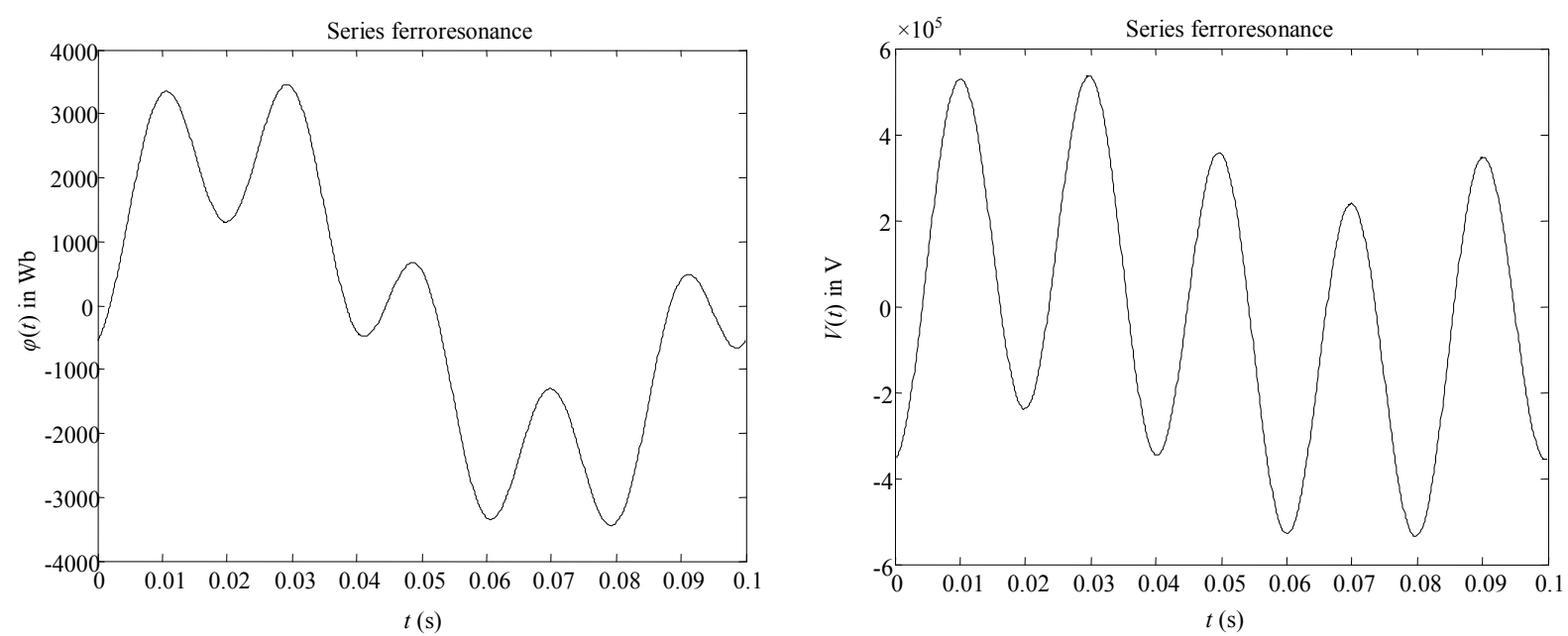

Figure 24. SH5 solution: Flux $\varphi(t)$ and voltage $V(t)$ waveforms on the target transformer during one period for the subharmonic-5 ferroresonance for $E=327 \mathrm{kV}$.
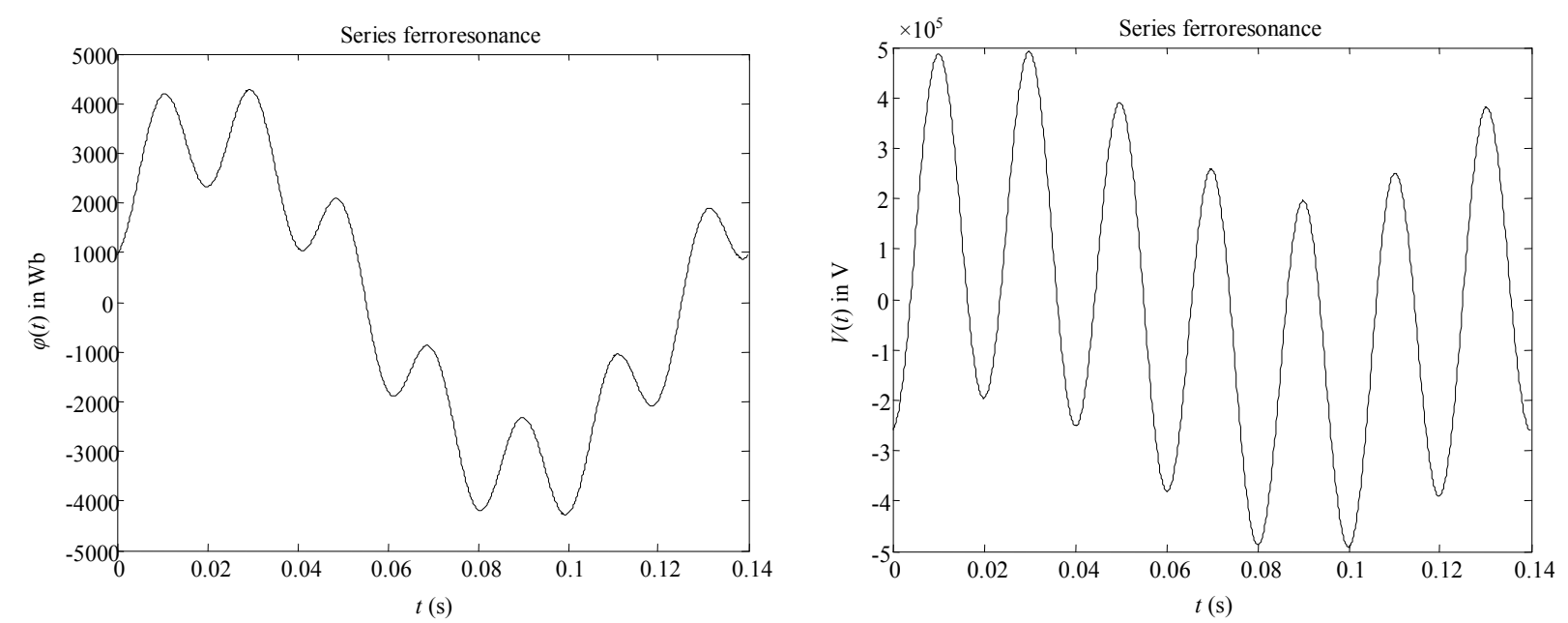

Figure 25. SH7 solution: Flux $\varphi(t)$ and voltage $V(t)$ waveforms on the target transformer during one period for the subharmonic-7 ferroresonance for $E=327 \mathrm{kV}$. 

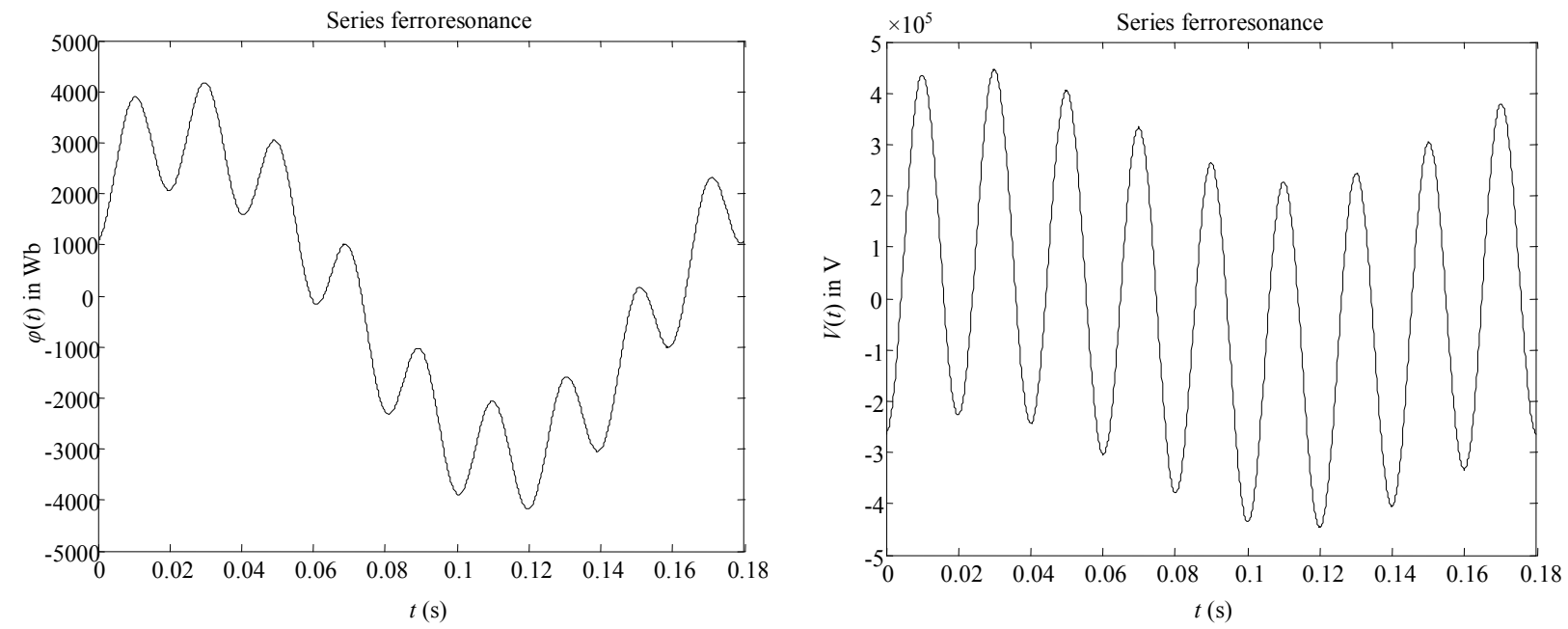

Figure 26. SH9 solution: Flux $\varphi(t)$ and voltage $V(t)$ waveforms on the target transformer during one period for the subharmonic-9 ferroresonance for $E=327 \mathrm{kV}$.
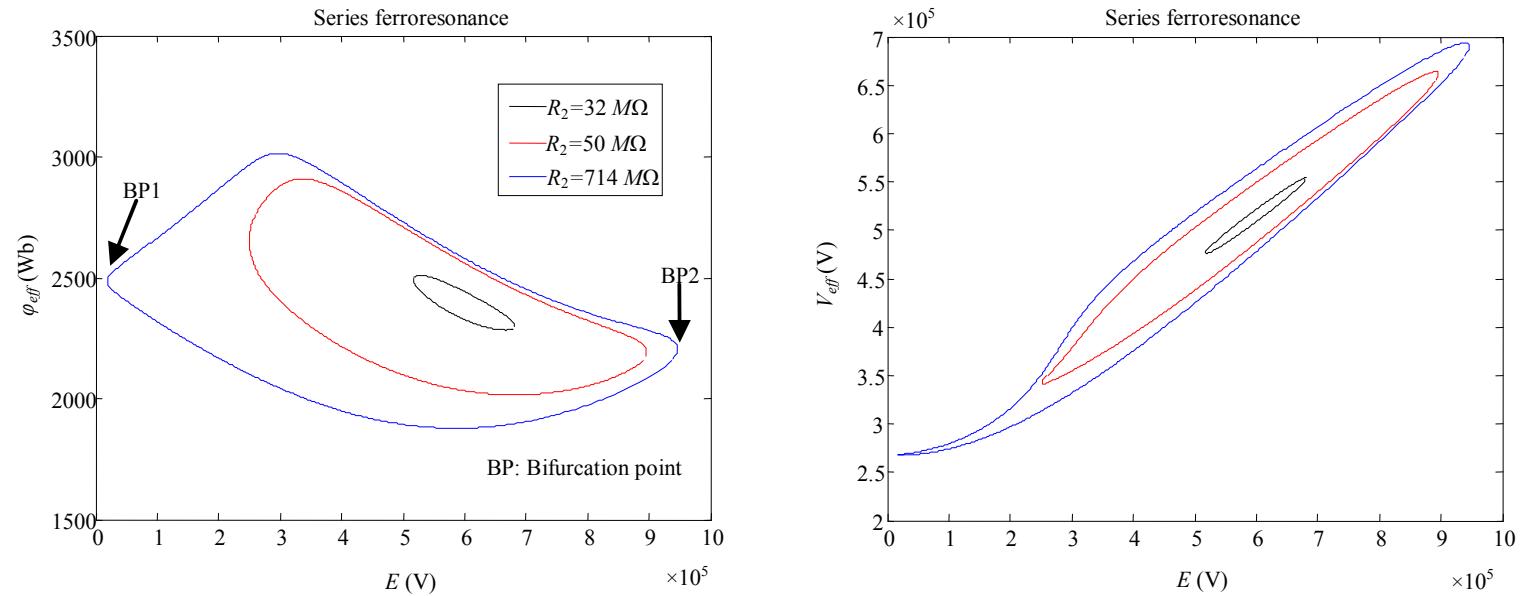

(a)
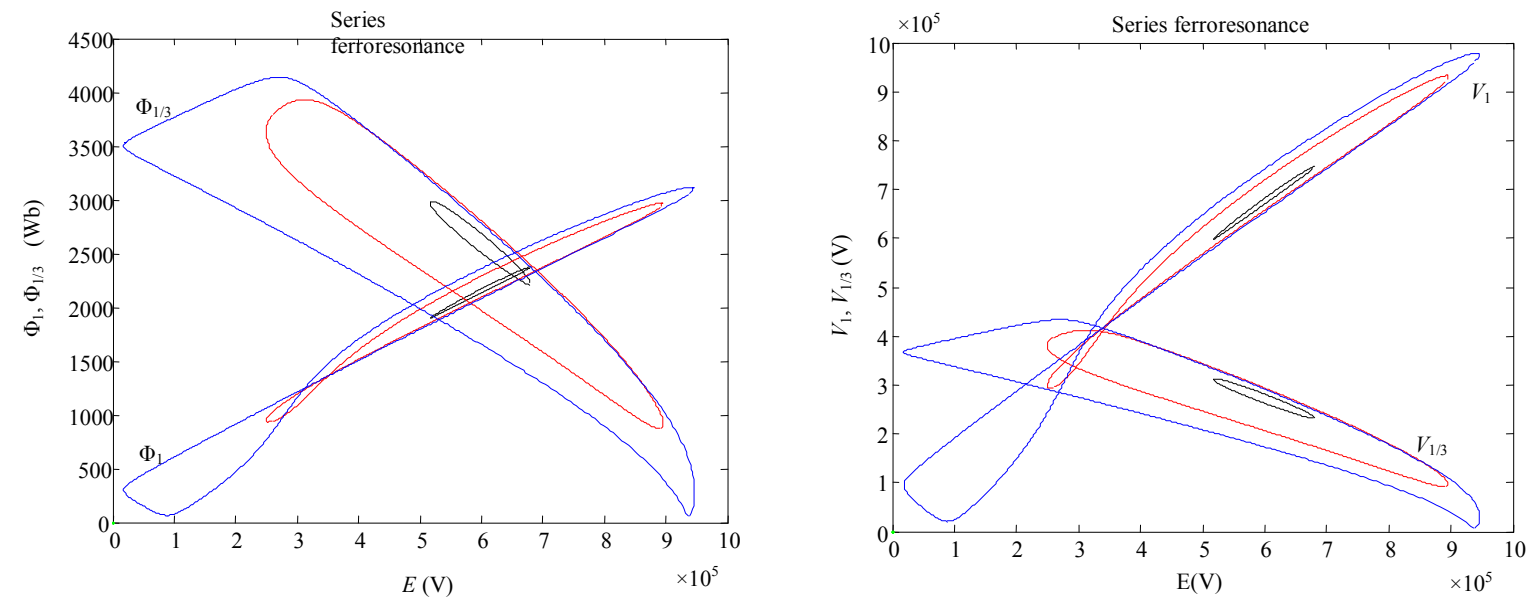

(b)

Figure 27. Bifurcation diagrams of SH3 mode as a function of $E$, for various values of the parallel losses $R_{2}\left(C_{t}=400 \mathrm{pF}, R_{1}=\right.$ $32 \mathrm{k} \Omega$ and $l=0 \mathrm{H}$ ). (a) Existence zones of the SH3 mode versus applied voltage $E$. (b) Continuation of the amplitudes of SH3 component and the $50 \mathrm{~Hz}$ component. 

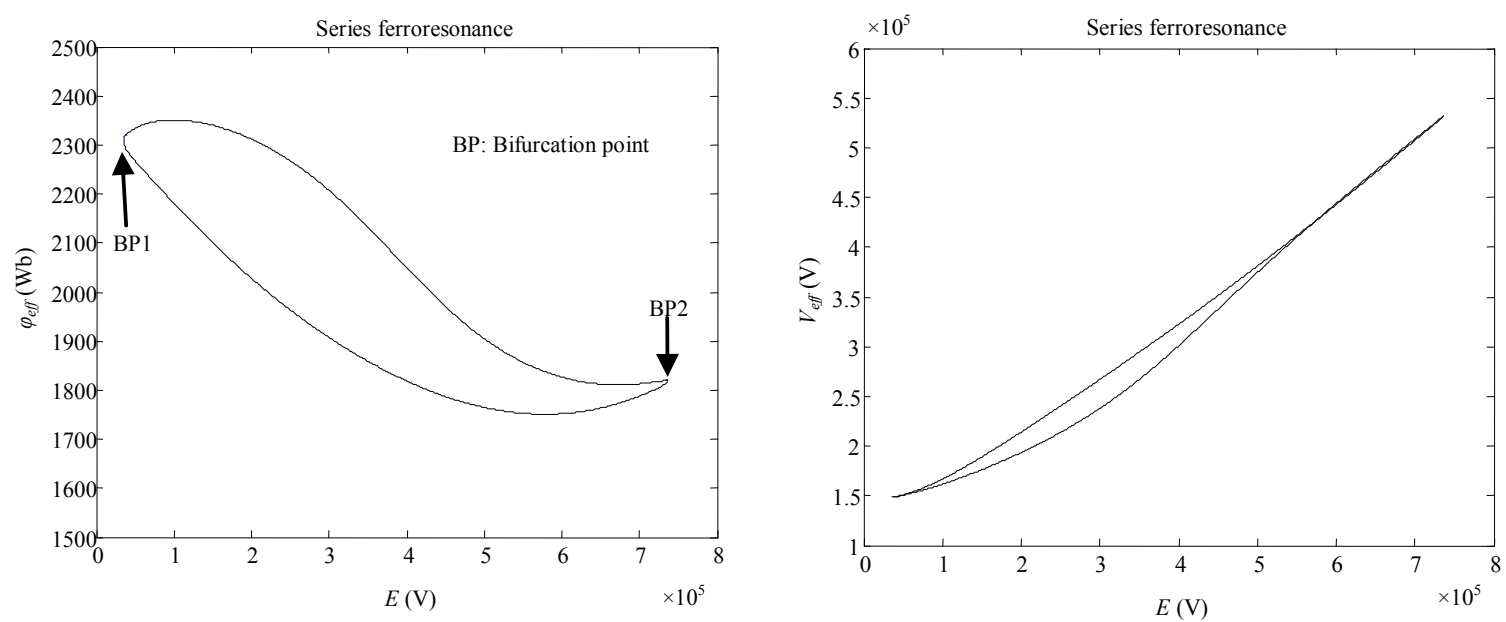

(a)
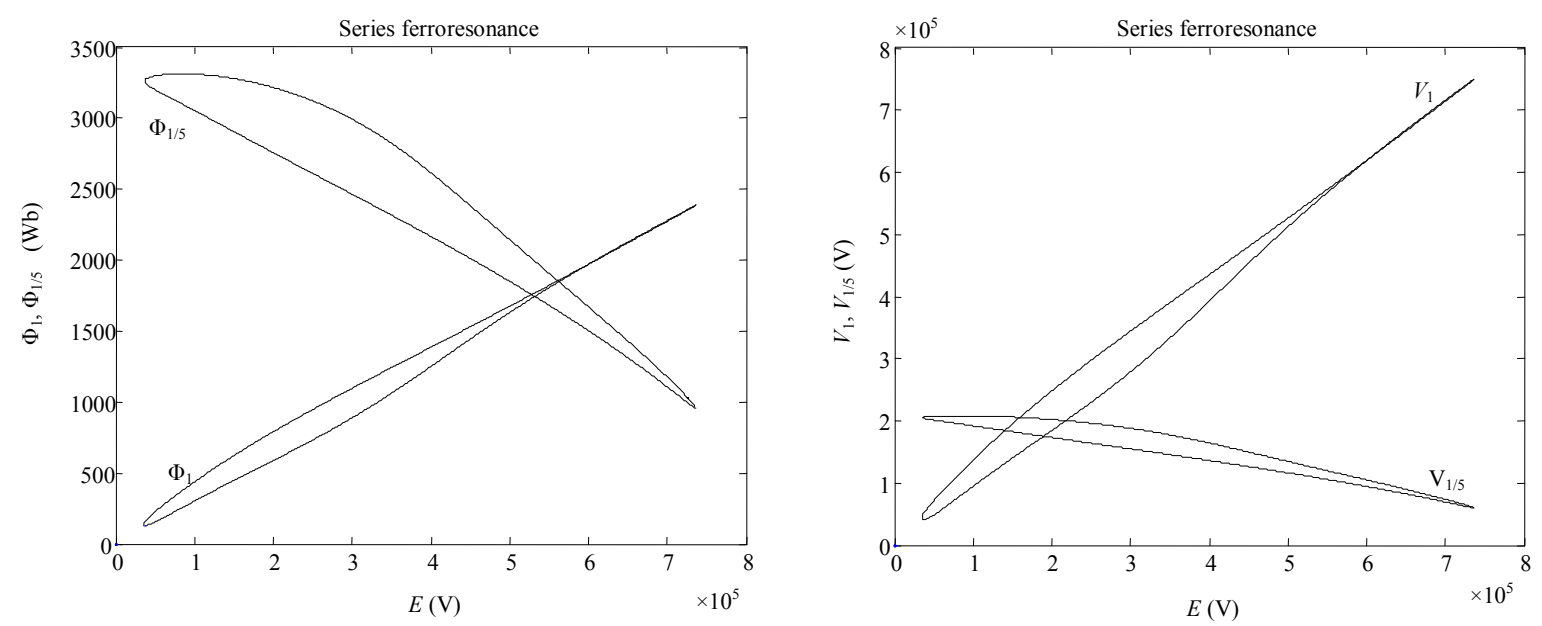

(b)

Figure 28. Bifurcation diagrams of SH5 mode as a function of $E$, for the circuit parameters: $R_{2}=714 \mathrm{M} \Omega, R_{1}=32 \mathrm{k} \Omega, C_{t}=$ $400 \mathrm{pF}$ and $l=0 \mathrm{H}$. (a) Existence zones of the SH5 mode versus applied voltage $E$. (b) Continuation of the amplitudes of SH5 component and the $50 \mathrm{~Hz}$ component.

\subsubsection{Search for Harmonics Modes}

We apply the method to the study of the harmonic modes 3, 5 and 7. Figures 34-36 give, over a period, the waveforms of their corresponding solutions. By examining these waves, we note that they respectively contain 3,5 and 7 maximums over a period; that means that the three solutions obtained are respectively very rich in harmonic $3(f=150 \mathrm{~Hz})$, in harmonic $5(f=250 \mathrm{~Hz})$ and in harmonic $7(f=350 \mathrm{~Hz})$. These three solutions are theoretical and do not represent any realistic situations for an electrical power network $\left(E \gg E_{\text {nom }}=327 \mathrm{kV}\right)$.

The continuation of these modes, as a function of the excitation voltage $E$, begins with the null trivial solution for $E=0$ and presents four bifurcation points $B P_{1}, B P_{2}$, $\mathrm{BP}_{3}$ and $\mathrm{BP}_{4}$ (Figures 37-39). For realistic voltage values, the mode can be normal or fundamental ferroresonance: the existence zone is delimited by the two singu- larities $B P_{1}$ and $B P_{2}$. However the studied harmonic modes are in a voltage interval which does not interest the power system operator (the nominal phase-to-neutral network voltage $230 \mathrm{kV}$ ). Their existence zones are delimited by the two singularities $\mathrm{BP}_{3}$ and $\mathrm{BP}_{4}$.

We compare the bifurcation diagrams of the harmonic 3, 5, 7 modes and of the fundamental mode (Figure 40), we find the same conclusions of the parallel ferroresonance. The bifurcation diagrams always start with the null trivial solution. The levels of flux $\varphi(t)$ and voltage $V(t)$, for the same circuit parameter, are always superior to those reached by fundamental ferroresonant mode. The source voltage applied is determining final mode. Indeed, while varying in a monotonous way the excitation voltage $E$, we observe in the increasing order the appearance of the various harmonic modes (even and odd). 
In all the treated examples, the ferroresonance phenomena appear only in the lightly loaded electrical networks.. In addition, it is necessary that these circuits are under the good initial conditions so that these phenomena are generated.

\section{Conclusions}

Ferrorresonance is a very complex nonlinear phenomenon which affects power transmission system and distribution system. It is a particular case of bifurcation of nonlinear dynamic system. We are thus interested in the initialization question of the continuations of several modes types.

We have presented in this study an analytical method for research from the periodic ferroresonant solutions.

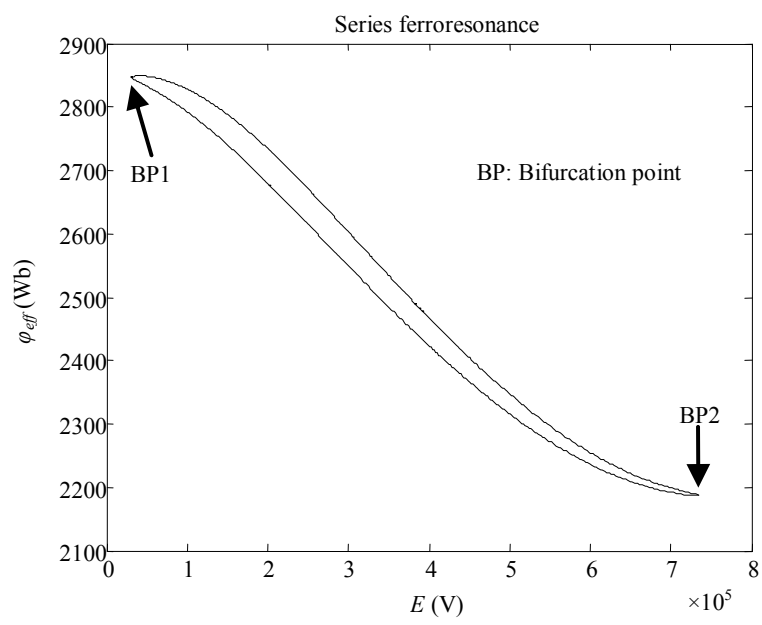

(a)

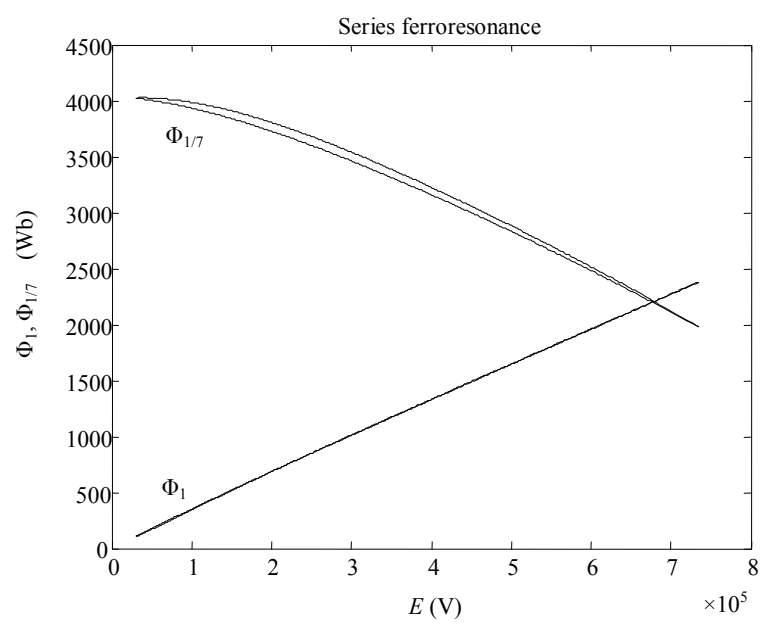

This approach enables us to systematically calculate the periodic modes intervening in the single-phase ferroresonance. The association of this method with a continuation technique enables to obtain the limits of the existence domains of the dangerous and undesirable modes as a function of source voltage $E$. We have presented, on real and theoretical configurations, parallel and series, the wealth of the informations brought by this analytical approach. Indeed, several results of continuation concerning the fundamental mode, harmonics $1,3,5$ and 7 modes and subharmonics 3, 5, 7, 9 and 3/5 modes are presented. It comes from this study the following overall conclusions:

- The risks of ferroresonant phenomena in the electrical power networks have an important appearance probability.
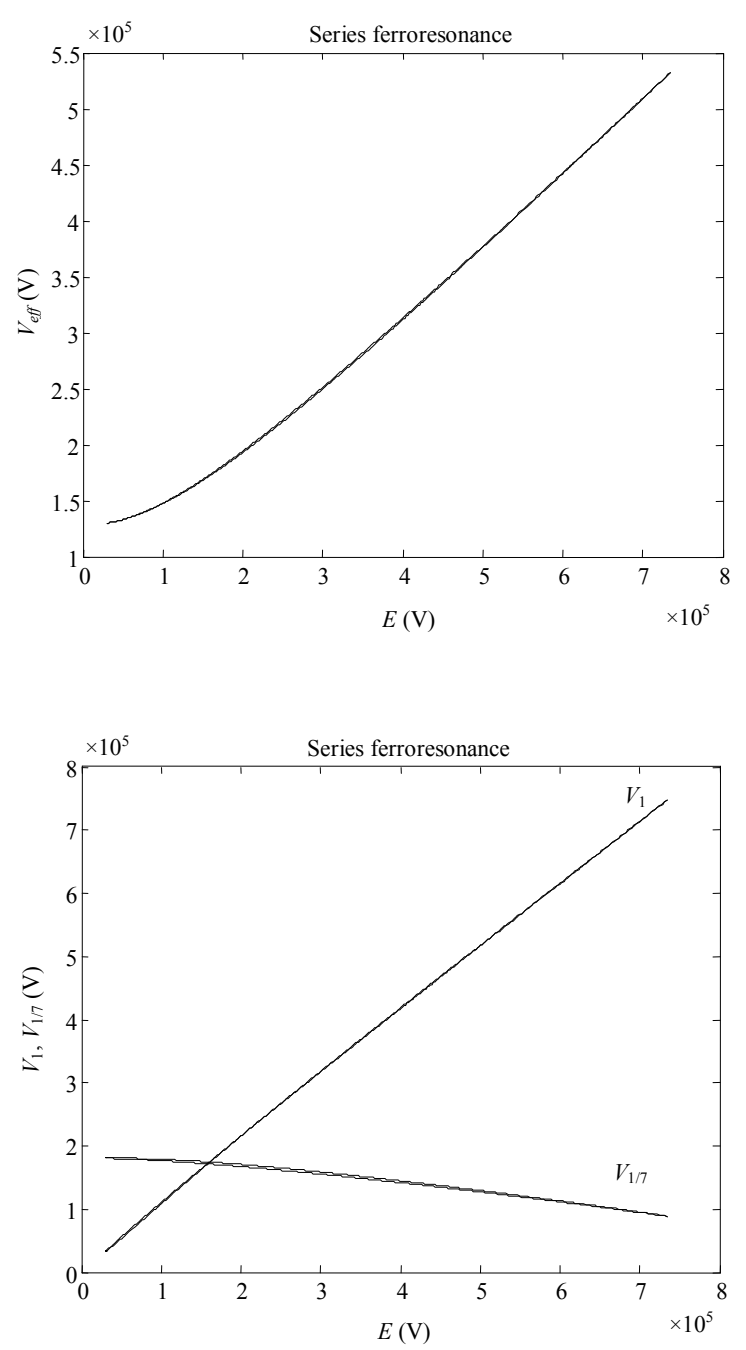

(b)

Figure 29. Bifurcation diagrams of $\mathrm{SH} 7$ mode as a function of $E$, for the circuit parameters: $R_{2}=714 \mathrm{M} \Omega, R_{1}=32 \mathrm{k} \Omega, C_{t}=4$ $\mathrm{nF}$ and $l=0 \mathrm{H}$. (a) Existence zones of the $\mathrm{SH} 7$ mode versus applied voltage $E$. (b) Continuation of the amplitudes of $\mathrm{SH} 7$ component and the $50 \mathrm{~Hz}$ component. 

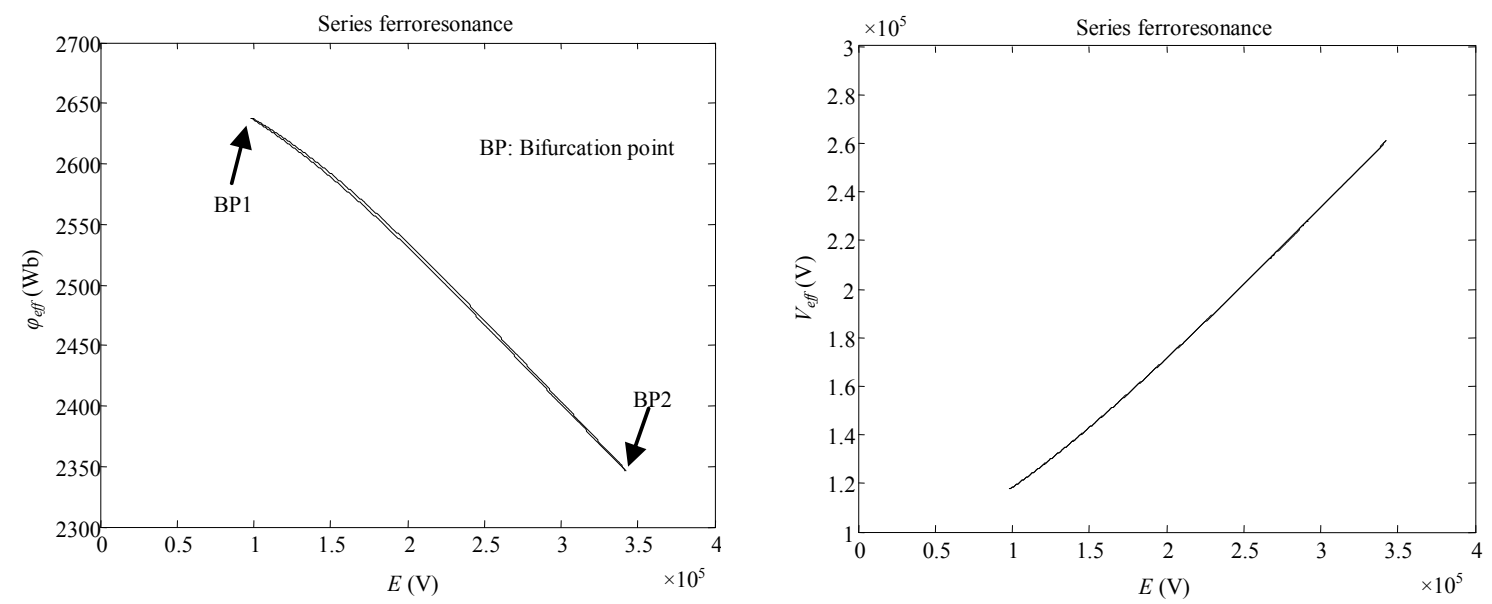

(a)
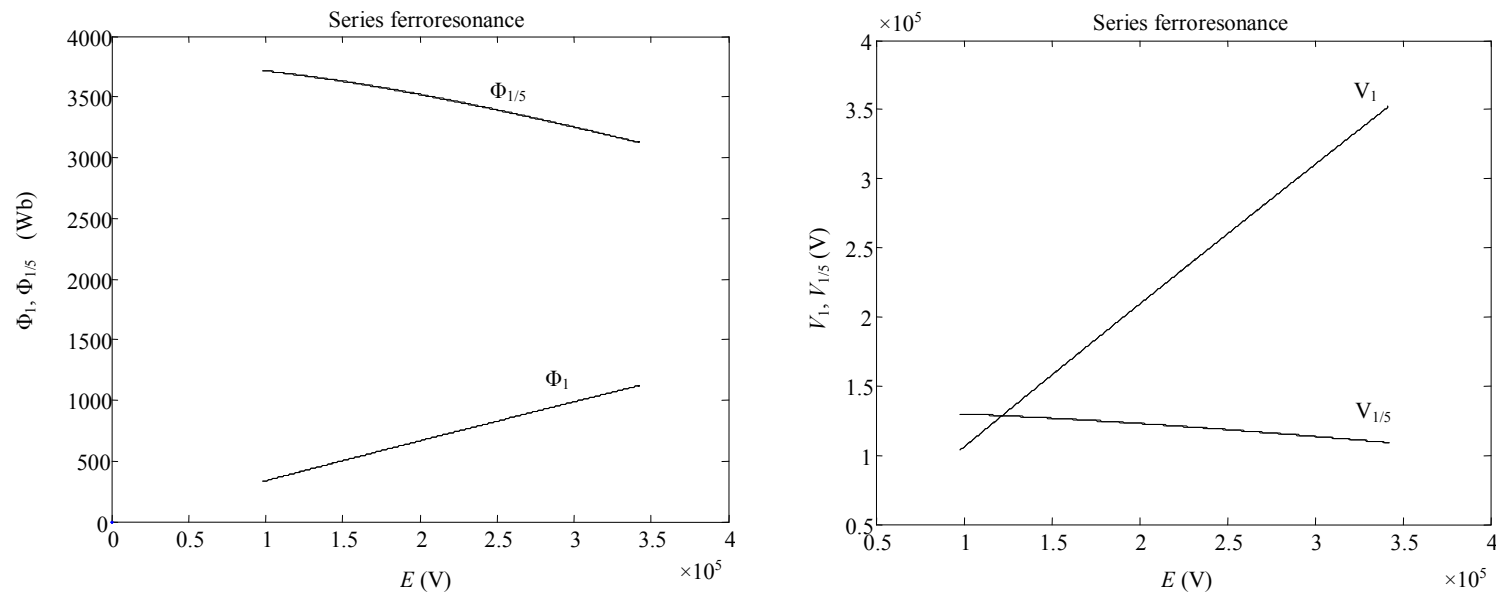

(b)

Figure 30. Bifurcation diagrams of $\mathrm{SH} 9$ mode as a function of $E$, for the circuit parameters: $R_{2}=7140 \mathrm{M} \Omega, R_{1}=32 \mathrm{k} \Omega, C_{t}=4$ nf and $l=0 \mathrm{H}$. (a) Existence zones of the SH9 mode versus applied voltage $E$. (b) Continuation of the amplitudes of SH9 component and the $50 \mathrm{~Hz}$ component.
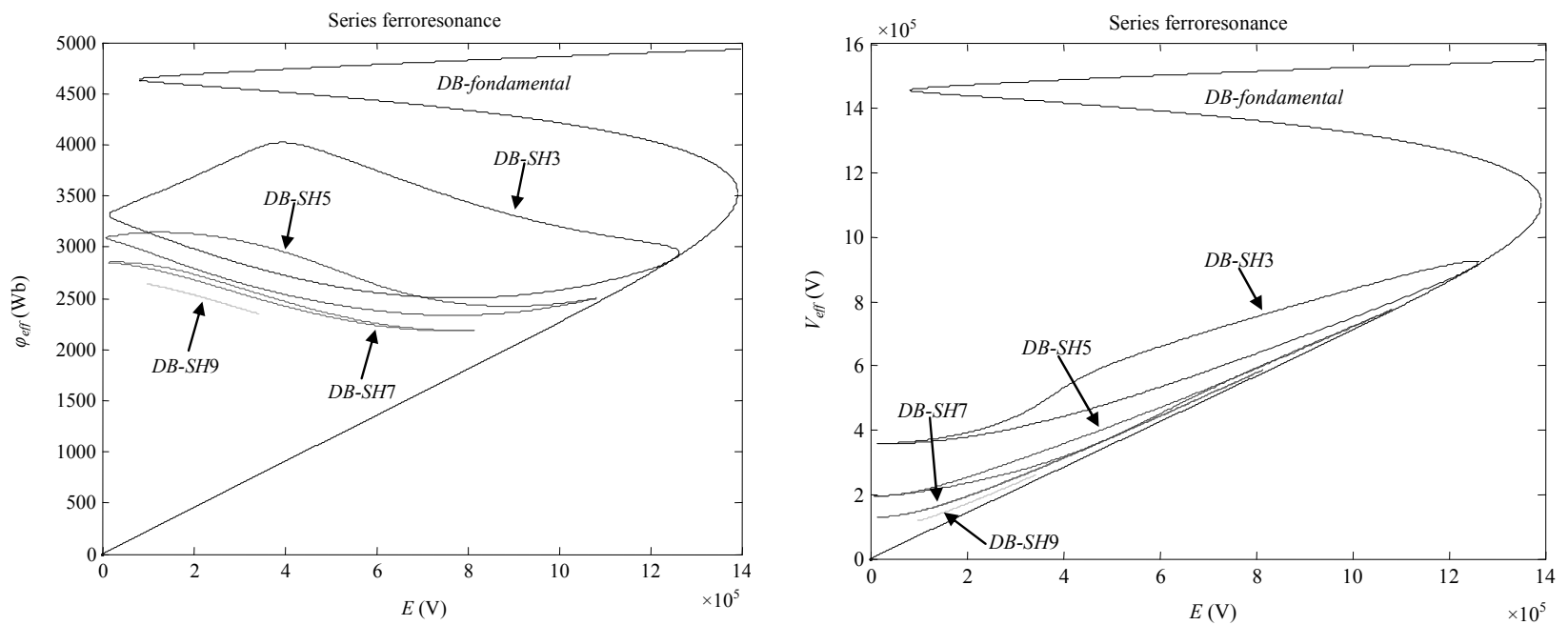

Figure 31. Comparison between bifurcation diagrams of SH3, 5, 7, 9 modes and of fundamental mode for the circuit parameters: $R_{2}=714 \mathrm{M} \Omega, R_{1}=32 \mathrm{k} \Omega, C=400 \mathrm{pF}$ and $l=0 \mathrm{H}$. 

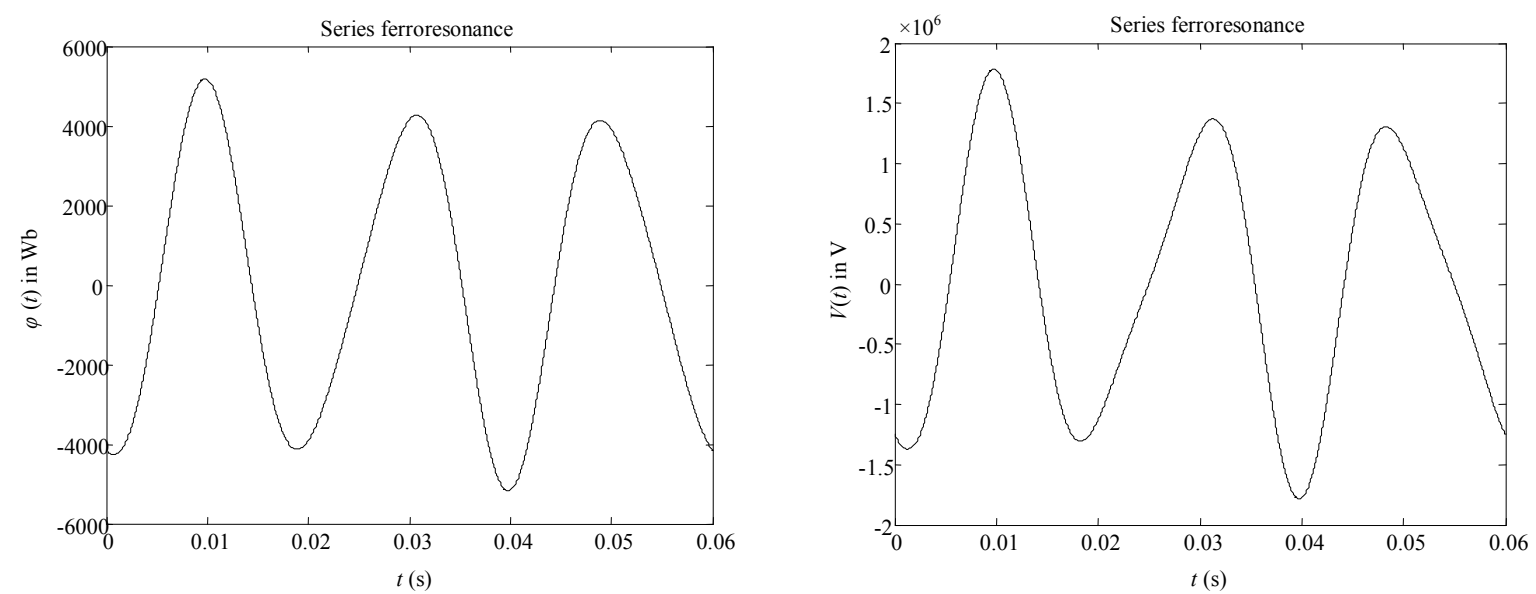

Figure 32. SH3/5 solution: Flux $\varphi(t)$ and voltage $V(t)$ waveforms on the target transformer during one period for the fractional subharmonic-3/5 ferroresonance for $E=327 \mathrm{kV}$.
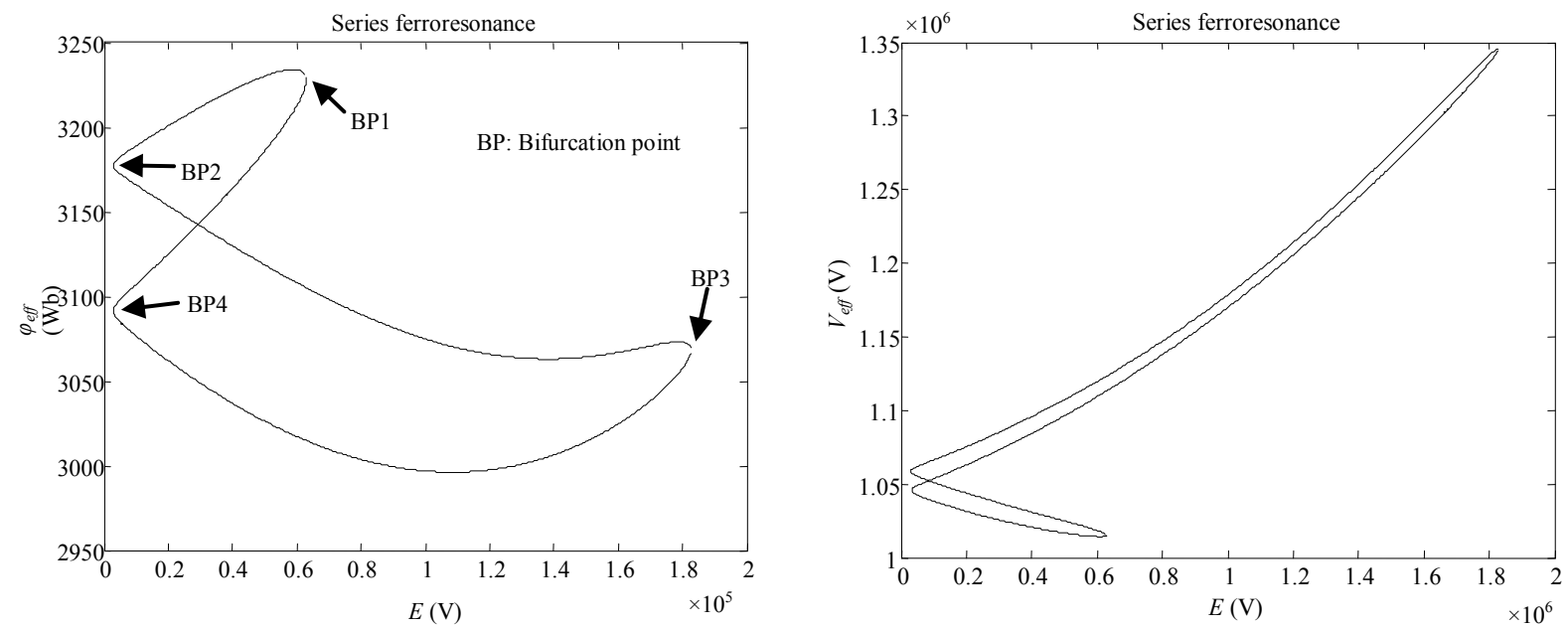

(a)
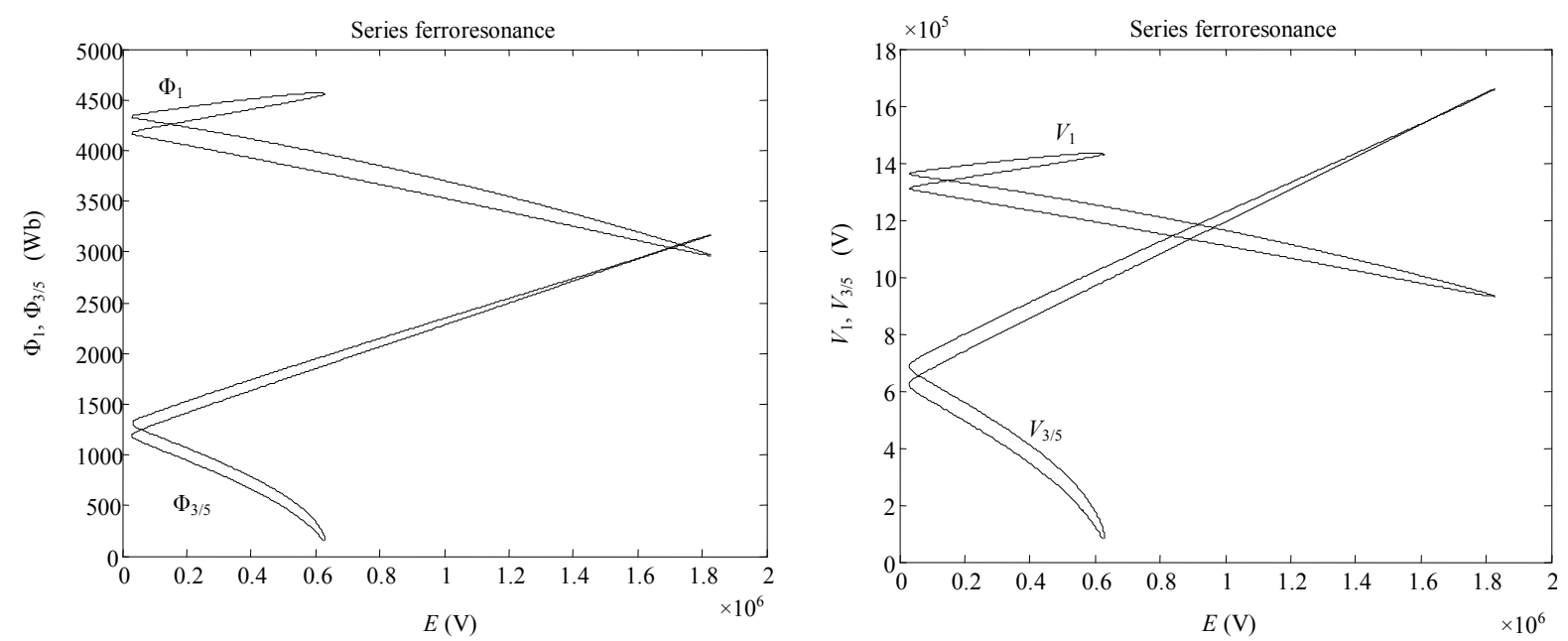

(b)

Figure 33. Bifurcation diagrams of $\mathrm{SH} 3 / 5$ mode as a function of $E$, for the circuit parameters: $R_{2}=714 \mathrm{M} \Omega, R_{1}=32 \mathrm{k} \Omega, C_{t}=$ $400 \mathrm{pF}$ and $l=0 \mathrm{H}$. (a) Existence zones of the $\mathrm{SH} 3 / 5$ mode versus applied voltage $E$. (b) Continuation of the amplitudes of $\mathrm{SH} 3 / 5$ component and the $50 \mathrm{~Hz}$ component. 


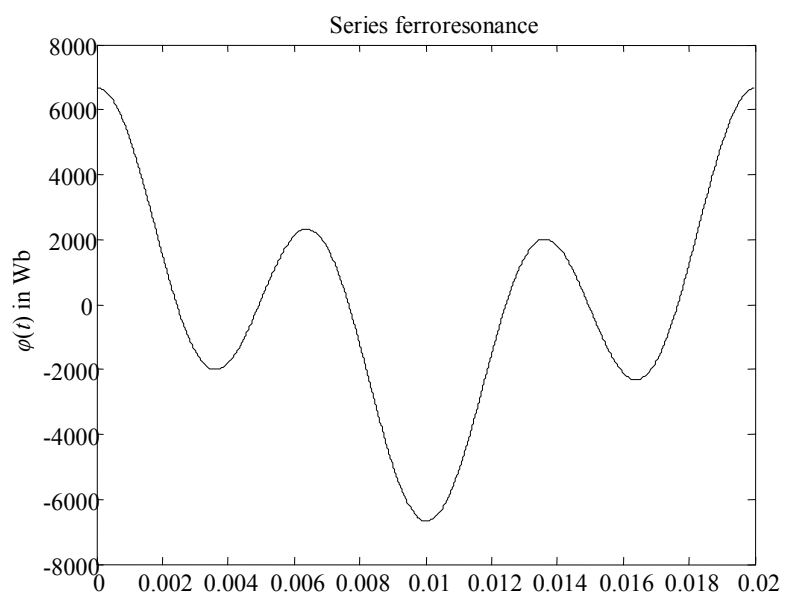

$t(\mathrm{~s})$

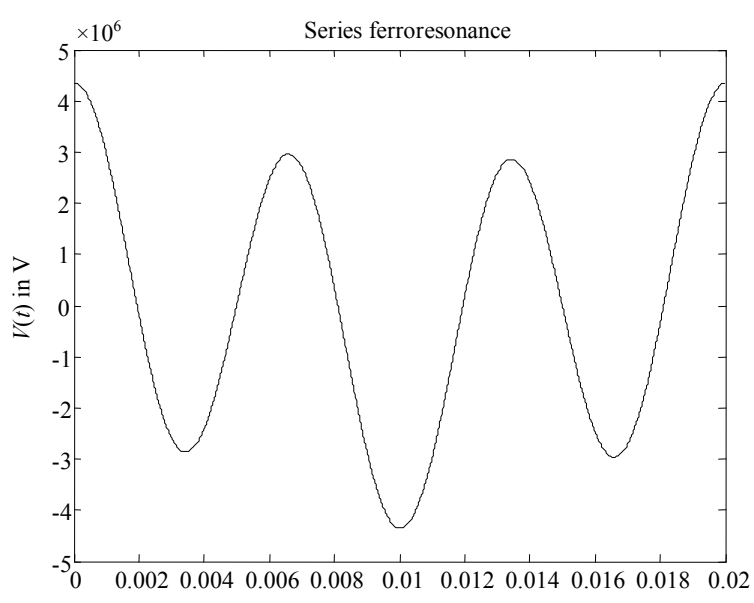

$t$ (s)

Figure 34. H3 solution: Flux $\varphi(t)$ and voltage $V(t)$ waveforms on the target transformer during one period for the harmonic-3 ferroresonance for $E=10 \mathrm{MV}$.

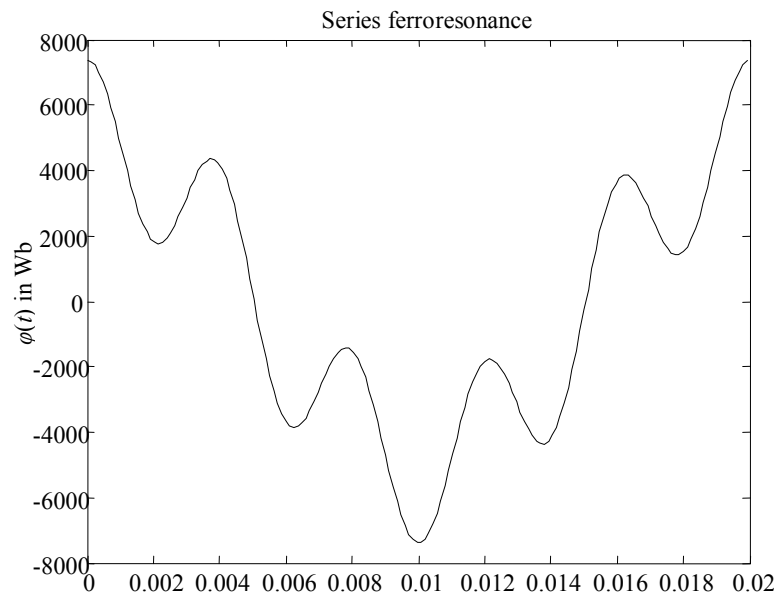

$t(\mathrm{~s})$

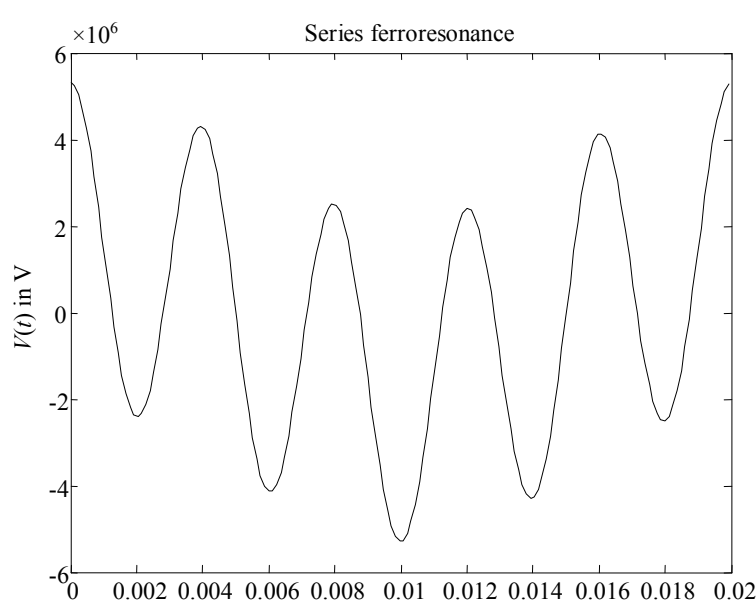

$t(\mathrm{~s})$

Figure 35. H5 solution: Flux $\varphi(t)$ and voltage $V(t)$ waveforms on the target transformer during one period for the harmonic-5 ferroresonance for $E=20 \mathrm{MV}$.

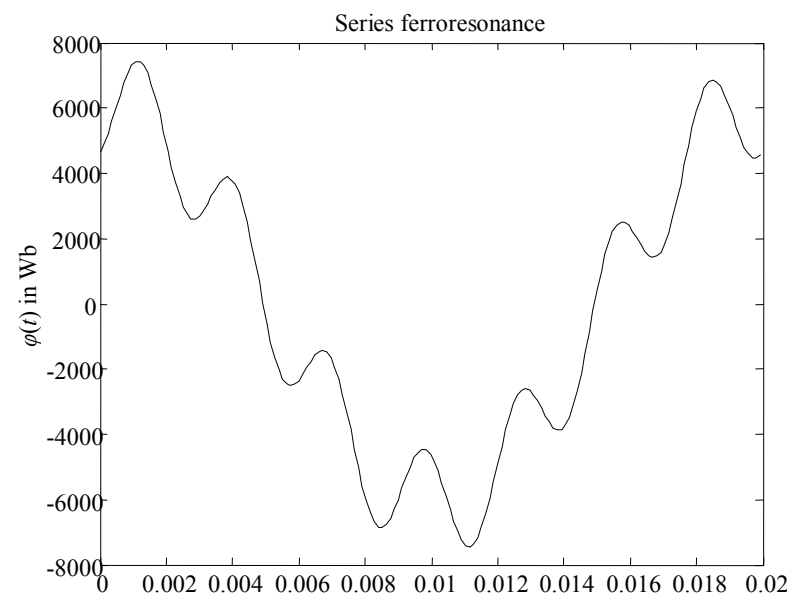

$t$ (s)

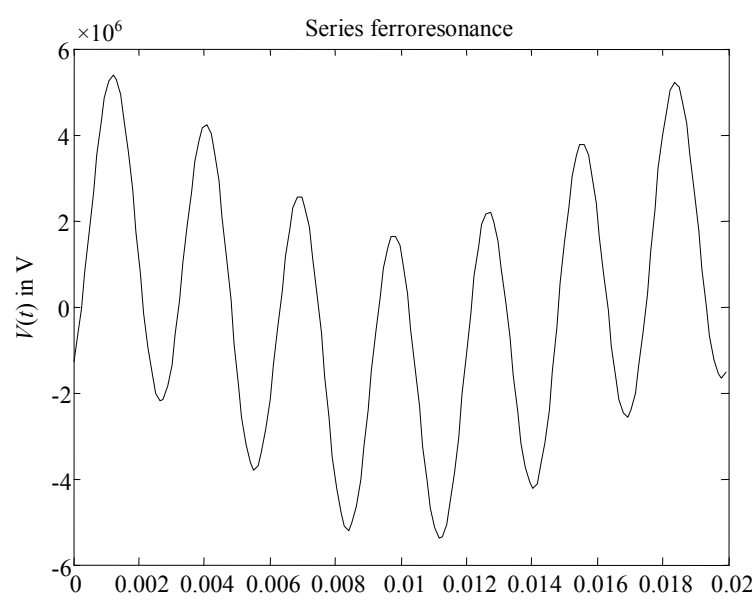

$t$ (s)

Figure 36. H7 solution: Flux $\varphi(t)$ and voltage $V(t)$ waveforms on the target transformer during one period for the harmonic-7 ferroresonance for $E=30 \mathrm{MV}$. 

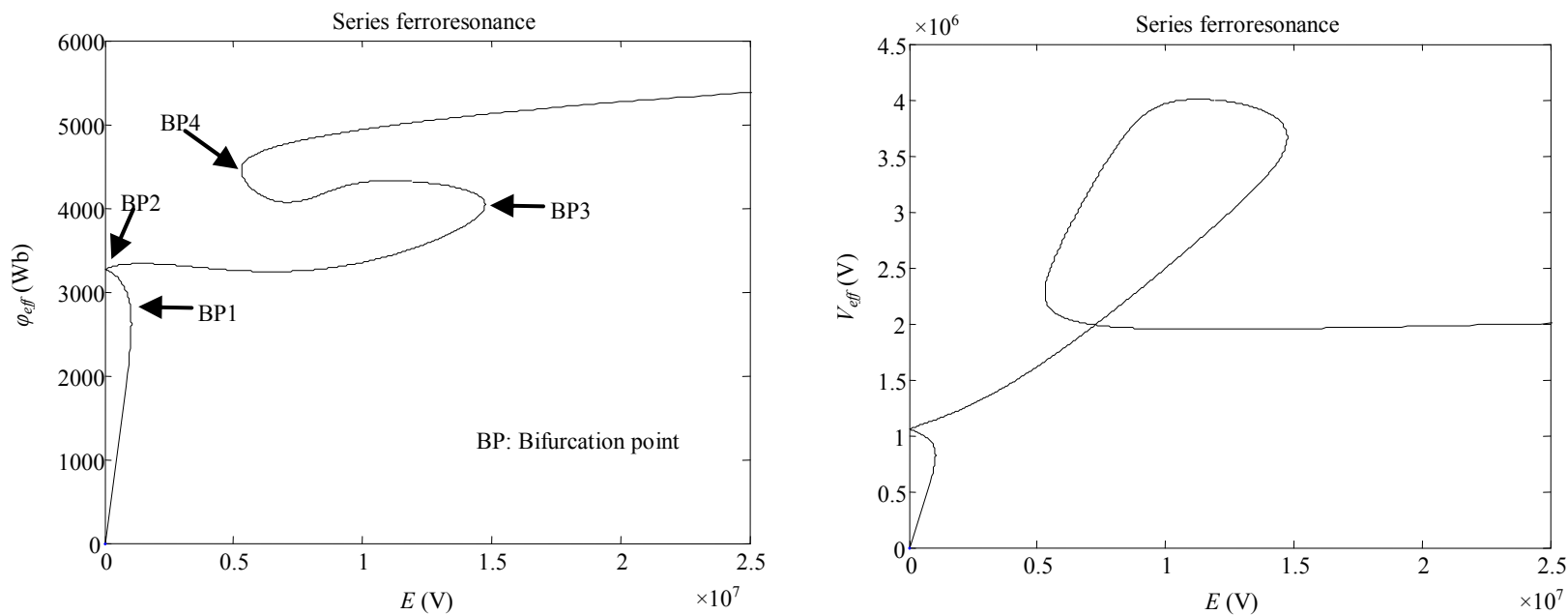

(a)
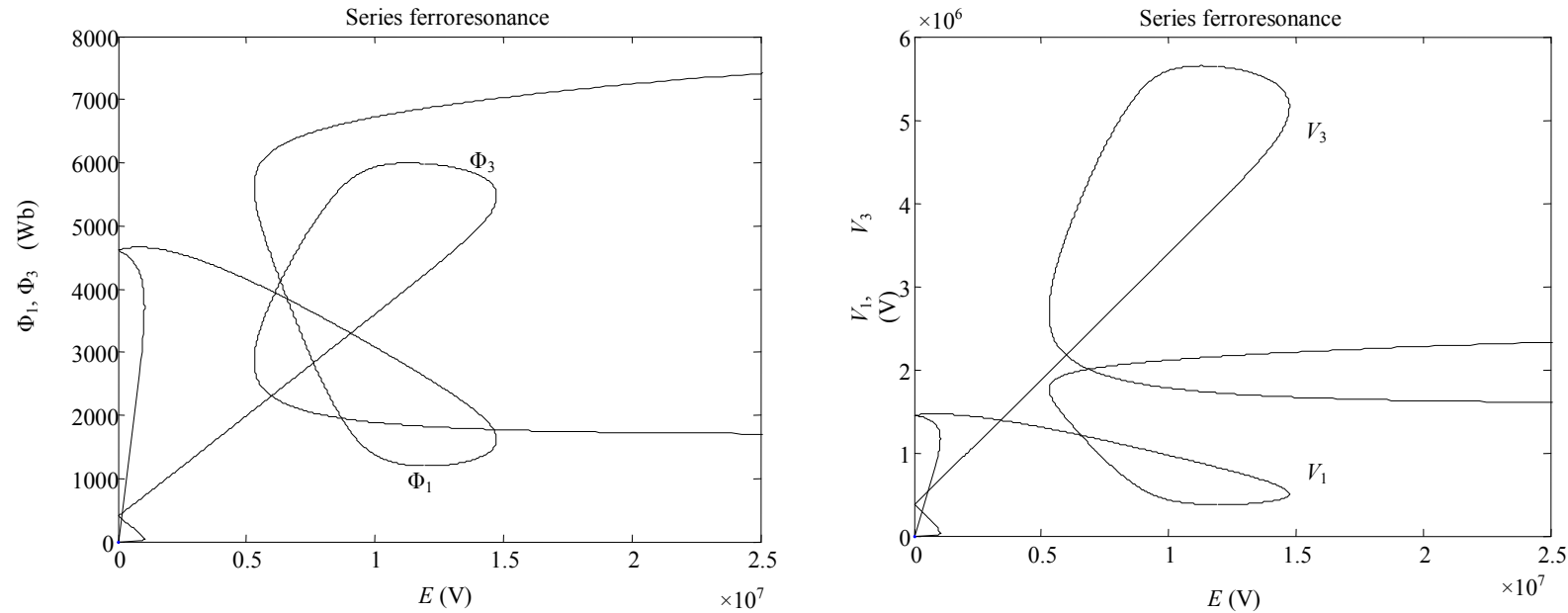

(b)

Figure 37. Bifurcation diagrams of $\mathrm{H3}$ mode as a function of $E$, for the circuit parameters: $R_{2}=714 \mathrm{M} \Omega, R_{1}=32 \mathrm{k} \Omega, C_{t}=400$ $\mathrm{pF}$ and $l=0 \mathrm{H}$. (a) Existence zones of the $\mathrm{H3}$ mode versus applied voltage $E$. (b) Continuation of the amplitudes of $\mathrm{H3} \mathrm{com}-$ ponent and the $50 \mathrm{~Hz}$ component.
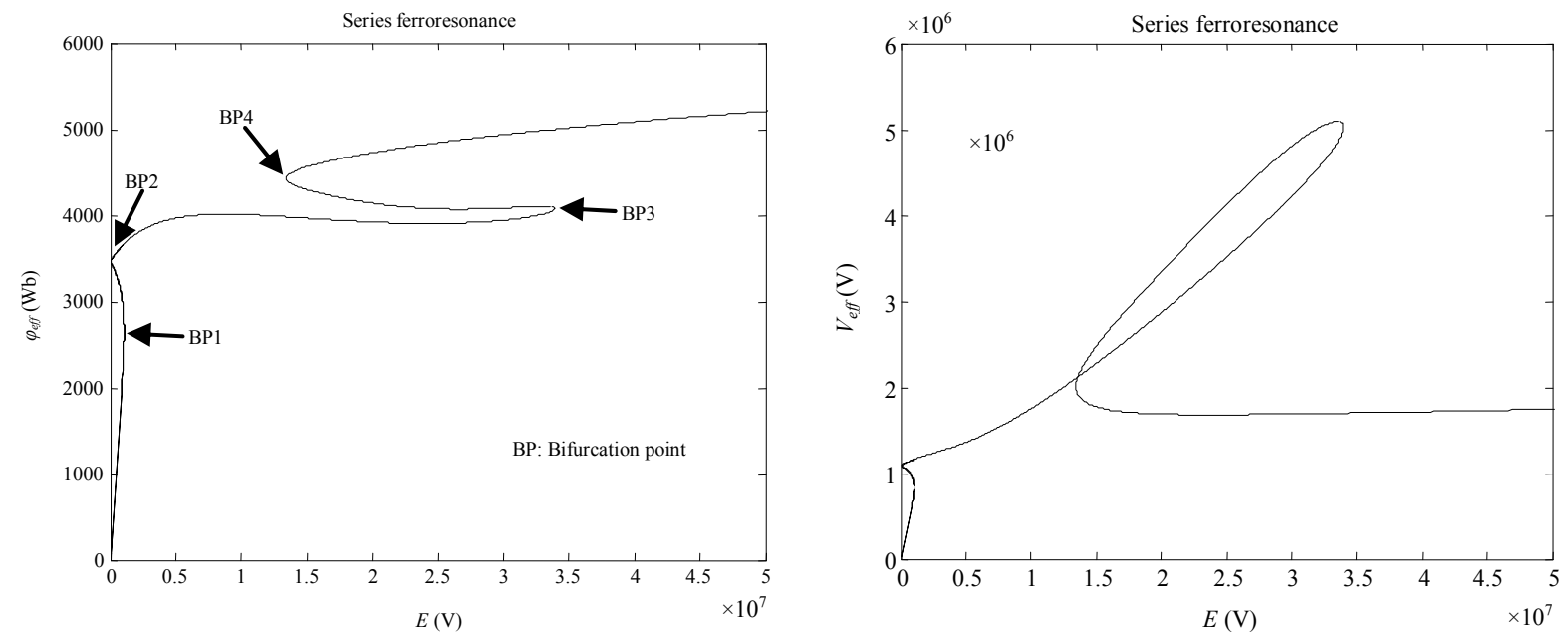

(a) 

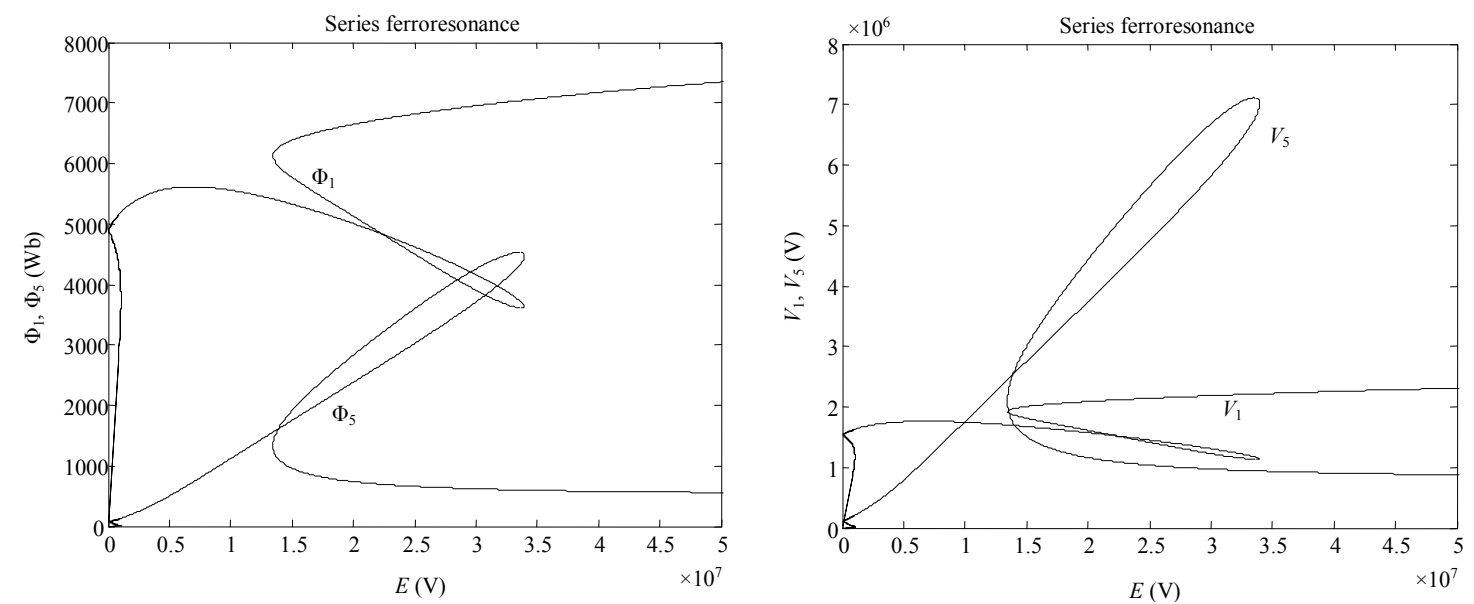

(b)

Figure 38. Bifurcation diagrams of $\mathrm{H5}$ mode as a function of $E$, for the circuit parameters: $R_{2}=714 \mathrm{M} \Omega, R_{1}=32 \mathrm{k} \Omega, C_{t}=400$ $\mathrm{pF}$ and $l=0 \mathrm{H}$. (a) Existence zones of the $\mathrm{H5}$ mode versus applied voltage $E$. (b) Continuation of the amplitudes of $\mathrm{H5}$ component and the $50 \mathrm{~Hz}$ component.
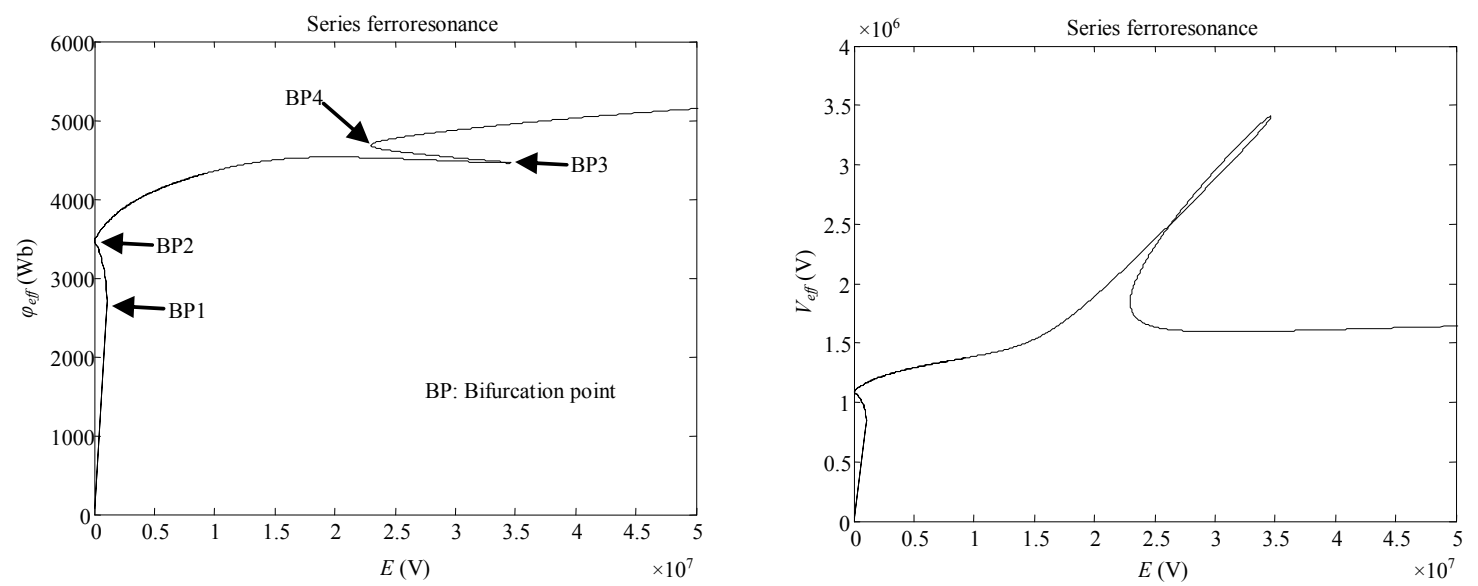

(a)
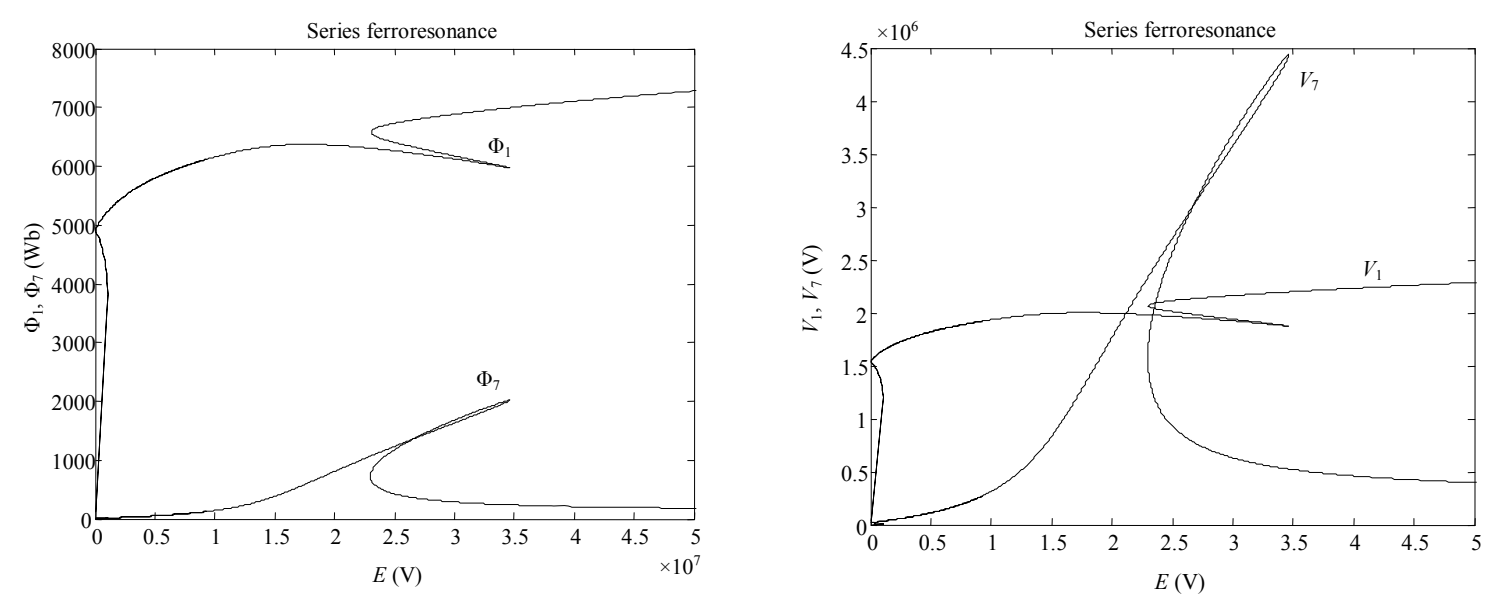

(b)

Figure 39. Bifurcation diagrams of $\mathrm{H} 7$ mode as a function of $E$, for the circuit parameters: $R_{2}=714 \mathrm{M} \Omega, R_{1}=32 \mathrm{k} \Omega, C_{t}=400$ $\mathrm{pF}$ and $l=0 \mathrm{H}$. (a) Existence zones of the $\mathrm{H} 7 \mathrm{mode}$ versus applied voltage $E$. (b) Continuation of the amplitudes of $\mathrm{H} 7 \mathrm{com}-$ ponent and the $50 \mathrm{~Hz}$ component. 

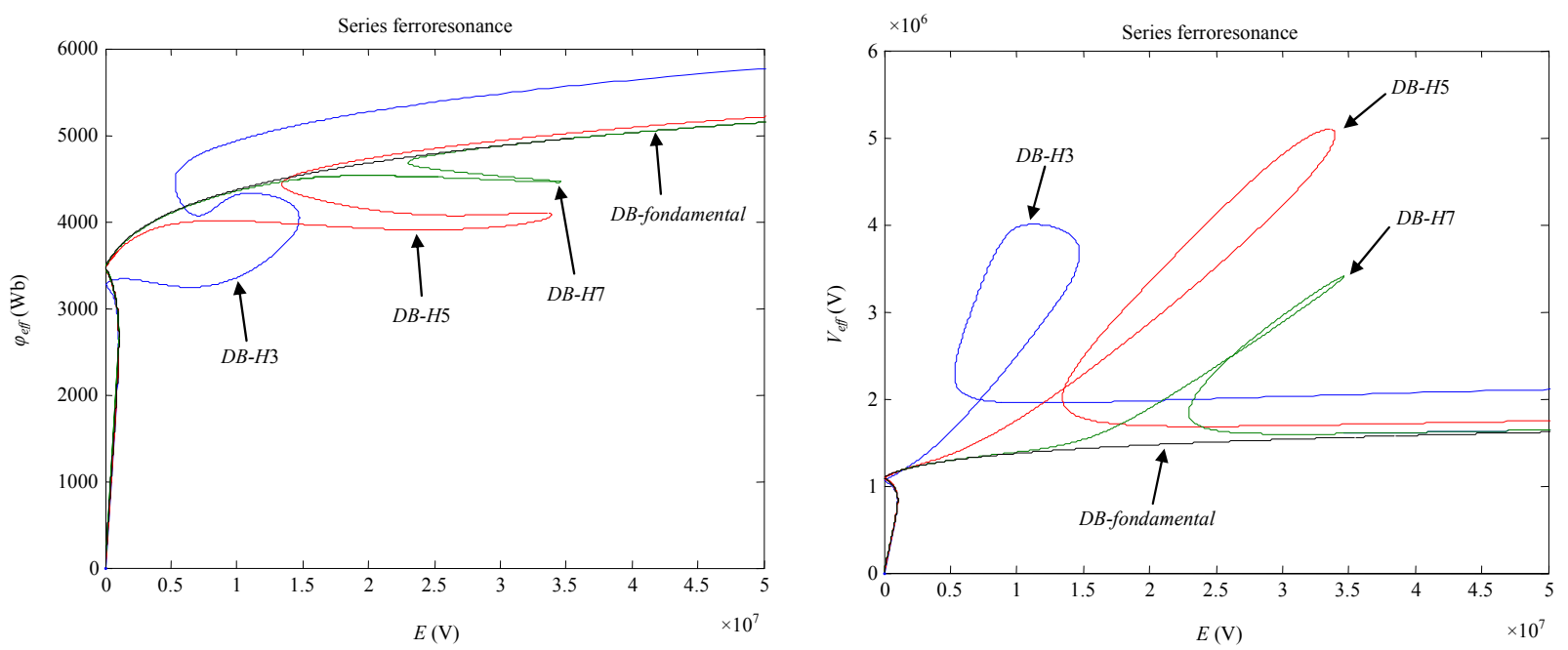

Figure 40. Comparison between bifurcation diagrams of $\mathbf{H 3}, 5,7$ modes and of fundamental mode for the circuit parameters: $R_{2}=714 \mathrm{M} \Omega, R_{1}=32 \mathrm{k} \Omega, C_{t}=400 \mathrm{pF}$ and $l=0 \mathrm{H}$.

- For all the series ferroresonances, the phenomenon can give rise to ferroresonance modes generally periodic of fundamental or subharmonic type. On the contrary, for all the parallel ferroresonances, the modes are harmonic type, i.e. fundamental with strong harmonic amplitudes in the spectrum, but also pseudoperiodics.

On the practical plane, the approach proposed is a tool enabling to determine if, in existing and future installation, there is danger of ferroresonance. It enables to predict and appraise the ferroresonance possibilities in an electrical network for the set of the source voltage values $E$ in normal and downgraded conditions.

The analytical approach developed with two components of flux gives us a first estimate of the solution and constitutes, in general, an excellent starting point for the iterations of the Galerkin method: a method which offers solutions closer to reality, owing to the fact that it does not impose any restriction on the component count of flux. A study in this direction is presently being pursued.

\section{References}

[1] K. Pattanapakdee and C. Banmongkol, "Failure of Riser Pole Arrester Due to Station Service Transformer," International Conference on Power Systems Transients, Lyon, 4-7 June 2007.

[2] S. Santoso, R. C. Dugan and H. Peter, "Modeling Ferroresonance Phenomena in an Underground Distribution System," International Conference on Power Systems Transients (IPST'07) Lyon, 4-7 June 2007.

[3] R. C. Dugan, "Examples of Ferroresonance in Distribution Systems," IEEE Power Engineering Society General Meeting, Knoxville, 13-17 July 2003.

[4] Ph. Ferracci, "Ferroresonance," Cahier Technique, Group Schneider, 1998.
[5] C. Kieny, "Application of the Bifurcation Theory in Studying and Understanding the Global Behaviour of a Ferroresonant Electric Power Circuit," IEEE Transactions on Power Delivery, Vol. 6, No. 2, 1991, pp. 866872. doi:10.1109/61.131146

[6] F. B. Amar and R. Dhifaoui, "Bifurcation Diagrams and Lines of Period-1 Ferroresonance," WSEAS Transactions on Power Systems, Vol. 1, No. 7, 2006, pp. 1154-1162.

[7] F. B. Amar and R. Dhifaoui, "Study of the Periodic Ferroresonance in the Electrical Power Networks by Bifurcation Diagrams," International Journal of Electrical Power and Energy Systems, Vol. 33, No. 1, 2011, pp. 61-85. doi:10.1016/j.ijepes.2010.08.003

[8] B. S. A. Kumar and S. Ertem, "Capacitor Voltage Transformer Induced Ferroresonance: Causes, Effects and Design Considerations," Electric Power Systems Research, Vol. 21, 1991, pp. 23-31.

[9] S. K. Chakravarthy and C. V. Nayar, "Series Ferroresonance in Power Systems," International Journal of Electrical Power \& Energy Systems, Vol. 17, No. 4, 1995, pp. 267-274. doi:10.1016/0142-0615(95)00045-R

[10] M. Rioual and C. Sicre, "Energization of a No-Load Transformer for Power Restoration Purposes: Impact of the Sensitivity to Parameters," International Conference on Power Systems Transients (IPST'01), Seattle, Vol. 2, 16 July 2001, pp. 892-895.

[11] N. Janssens, V. Vanderstockt, H. Denoel and P. A. Monfils, "Elimination of Temporary Overvoltages Due to Ferroresonance of Voltage Transformers: Design and Testing of Damping System," International Council on Large Electric Systems, Paris, 1990, pp. 33-204.

[12] R. G. Kavasseri, "Analysis of Subharmonic Oscillations in a Ferroresonant Circuit," International Journal of Electrical Power and Energy Systems, Vol. 28, No. 3, 2005, pp. 207-214. doi:10.1016/j.ijepes.2005.11.010

[13] K. S. Preetham, R. Saravanaselvan and R. Ramanujam, "Investigation of Subharmonic Ferroresonant Oscillations in Power Systems," Electric Power Systems Research, 
Vol. 76, No. 9-10, 2006, pp. 873-879. doi:10.1016/i.epsr.2005.11.005

[14] R. G. Kavasseri, "Analysis of Subharmonic Oscillations in a Ferroresonant Circuit," International Journal of Electrical Power and Energy Systems, Vol. 28, No. 3, 2005, pp. 207-214. doi:10.1016/j.ijepes.2005.11.010

[15] M. V. Escudero, I. Dudurych and M. A. Redfern, "Characterization of Ferroresonant Modes in HV Substation with CB Grading Capacitors," Electric Power Systems Research, Vol. 77, No. 11, 2007, pp. 1506-1513. doi:10.1016/j.epsr.2006.08.033

[16] S. Mitrea and A. Adascalitei, "On the Prediction of Ferroresonance in Distribution Networks," Electric Power Systems Research, Vol. 23, No. 2, 1992, pp. 155-160. doi:10.1016/0378-7796(92)90063-7

[17] M. M. Saied, H. M. Abdallah and A. S. Abdallah, "Damping Effect of Load on the Ferroresonance Phenomenon in Power Networks," Electric Power Systems Research, Vol. 7, 1984, pp. 271-277. doi:10.1016/0378-7796(84)90011-7 